\title{
Heterogeneous Reservoirs in the Marine Carbon Cycle
}

by

\author{
Christopher L. Follett
}

B.S., Massachusetts Institute of Technology, 2007

Submitted in partial fulfillment of the requirements for the degree of

Doctor of Philosophy

at the

MASSACHUSETTS INSTITUTE OF TECHNOLOGY

and the

\section{WOODS HOLE OCEANOGRAPHIC INSTITUTION}

June 2014

(C)2014 Christopher L. Follett. All rights reserved.

The author hereby grants to MIT and WHOI permission to reproduce and to distribute publicly paper and electronic copies of this thesis document in whole or in part in any medium known or hereafter created.

Author

Joint Program in Oceanography Massachusetts Institute of Technology and Woods Hole Oceanographic Institution March 14, 2014

Certified by .

Daniel H. Rothman

Professor of Earth, Atmospheric, and Planetary Sciences Thesis Supervisor Accepted by

Elizabeth B. Kujawinski Chair, Joint Committee for Chemical Oceanography Woods Hole Oceanographic Institution 


\title{
Heterogeneous Reservoirs in the Marine Carbon Cycle \\ by

\author{
Christopher L. Follett
}

\author{
Submitted to the Joint Program in Oceanography \\ Massachusetts Institute of Technology and Woods Hole Oceanographic Institution \\ on March 14, 2014, in partial fulfillment of the \\ requirements for the degree of \\ Doctor of Philosophy
}

\begin{abstract}
Understanding the fate of primary production in the ocean is a challenging task because once produced, organic material is oxidized over timescales which range from minutes, to millions of years. This timescale diversity is matched by an equal heterogeneity in both the local physical and chemical environment. In this thesis we explore the relationship between the distinct reservoirs of organic carbon in the ocean and their underlying complexity. First, we show how the heterogeneity of portions of the carbon cycle can be packaged in terms of age structured models and their accompanying age and rate distributions. We further relate the moments of the rate distributions to bulk reservoir properties like average age and flux. Explicit relationships are then derived for the specific case of a single turnover time and a lognormal distribution. We apply these ideas to the problem of dissolved organic carbon (DOC) cycling in the ocean. Current models of bulk concentration and isotope data suggest a microbially sourced DOC reservoir consisting of two components. A nearly homogeneous background component with a long turnover time ( $>6000$ years) is joined by a component of fast turnover time $(\sim 1$ year $)$ and equal concentration in the surface ocean. We confirm the presence of isotopically enriched, modern DOC co-cycling with an isotopically depleted older fraction in the upper ocean. However, our results show that up to $30 \%$ of the deep DOC reservoir is modern and supported by a $1 \mathrm{Pg}$ per year carbon flux, ten times higher than inferred from bulk isotope measurements. Isotopically depleted material turns over at an apparent time scale of 30,000 years, far slower than indicated by bulk isotope measurements. These results are consistent with global DOC measurements and explain both the fluctuations in deep DOC concentration and the anomalous radiocarbon values of DOC in the Southern Ocean. Finally, the thesis explores methods for determining the validity of diffusion limitation as the mechanism behind the power-law slowdown in organic remineralization in sediment. We find that diffusion limitation connects the decay behavior of organic material to the correlations found between mineral surface area and organic matter content in sediments.
\end{abstract}

Thesis Supervisor: Daniel H. Rothman

Title: Professor of Earth, Atmospheric, and Planetary Sciences 


\section{Acknowledgments}

I have wanted to be an oceanographer for an extremely long time and am forever indebted to the many opportunities afforded to me along the way, the people I have met, and the kind words they put in for me. I want to thank my advisors for their continued support, and help in bringing some of our ideas to a productive conclusion. On a personal note, I have always enjoyed the full support of my parents and sister who must have on some occasions wondered why my passion was oceanography. Lastly, my wife and daughter have put up with me through this process, and provided me with continual joy through the ups and downs. I benefitted from the support of both a Linden Fellowship, and the Jule Charney Prize at MIT. This work was funded by National Science Foundation grants OCE-0930866 and EAR-0420592 on which Daniel Rothman was the primary investigator. Additional work was funded by National Science Foundation Science and Technology Center for Microbial Oceanography Research and Education (C-MORE) grant number CCF-424599 and the Gordon and Betty Moore Foundation Microbial Oceanography Initiative grant number 3298 for which Daniel Repeta (Woods Hole Oceanographic Institution) was the primary investigator. 


\section{Contents}

1 Introduction 11

2 Statistical Kinetics of the Marine Carbon Cycle 19

2.1 Introduction . . . . . . . . . . . . . . . . . 20

2.2 Age-Structured Models . . . . . . . . . . . . . . . . . . . . 24

2.2.1 Equating Reservoir Properties with Age Structure . . . . . . 27

2.2.2 The Lognormal Rate Distribution . . . . . . . . . . . . . 30

2.2.3 Application to Dissolved Organic Carbon . . . . . . . . . . . 31

2.3 Heterogeneity and Bulk Measurements . . . . . . . . . . . . 36

2.3.1 Equating Radiocarbon Age with Flux in a First Order Reservoir 37

2.3.2 Measuring the Turnover Time Distribution . . . . . . . . . . 39

2.4 Final Thoughts . . . . . . . . . . . . . . . . . . . . . 43

3 Is Dissolved Organic Carbon in the Deep Ocean in a Dynamic Steady $\begin{array}{ll}\text { State? } & 45\end{array}$

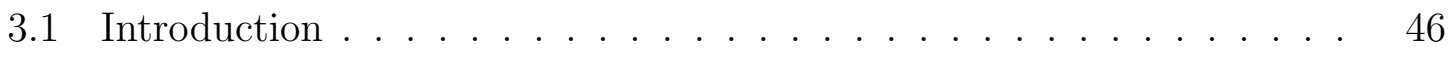

3.2 Bulk Concentration Data . . . . . . . . . . . . . . . . . . 52

3.2 .1 Changes in Local Variability . . . . . . . . . . . . . . 54

3.2 .2 Application of Random Inputs . . . . . . . . . . . . . . . 59

3.3 Radiocarbon Data . . . . . . . . . . . . . . . 60

3.3.1 Curvature in Keeling Space _. . . . . . . . . . . . . 61 
3.3.2 Spatial Variability of Keeling Slope . . . . . . . . . . . . 75

3.3.3 Anomalous DOC Aging in the Southern Ocean . . . . . . 77

3.3.4 Direct Estimation of Equilibration Timescale from Bomb-spike Information . . . . . . . . . . . . . . . 81

3.3.5 Constraining Deep Water DOC with a Simple Box Model . . . 82

3.4 Final Thoughts . . . . . . . . . . . . . . . . . . 86

3.5 Supporting Information . . . . . . . . . . . . . . . . . . . . 89

3.5.1 Derivation of Keeling Line . . . . . . . . . . . . . . 89

3.5.2 Example of Keeling Errors Affecting the Mean . . . . . . . . 90

4 Hidden Cycle of Dissolved Organic Carbon in the Deep Ocean 93

4.1 Introduction $\ldots \ldots \ldots \ldots \ldots \ldots \ldots \ldots \ldots \ldots$

4.2 The Isotopic Distribution within DOC $\ldots \ldots \ldots \ldots$

4.3 Radiocarbon and DOC Cycling . . . . . . . . . . . . . 100

4.4 Global Context . . . . . . . . . . . . . . . . . . 103

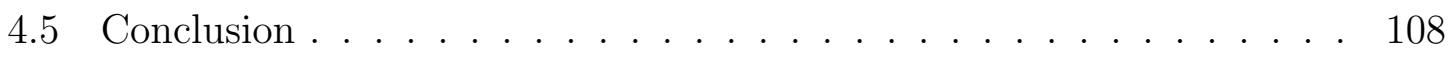

4.6 Supporting Information . . . . . . . . . . . . . . . . . 108

4.6.1 Materials and Methods . . . . . . . . . . . . . . . 108

4.6.2 DOC Photo-oxidation Kinetics ． . . . . . . . . . . 113

4.6.3 Estimating the Isotopic Distribution _ . . . . . . . . . . 115

4.6.4 Radiocarbon Turnover Time . . . . . . . . . . . . . . . 118

5 Heterogeneous Rate Distributions and their Effect on the Cycling $\begin{array}{ll}\text { of Organic Carbon in Sediments } & 121\end{array}$

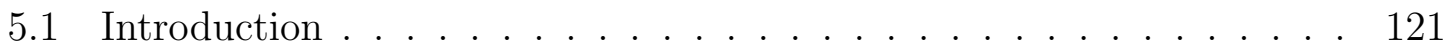

5.2 Theory: A Physical Model of Organic Matter Degradation . . . . . . 124

5.2.1 Linearity Between Organic Matter and Mineral Surface Area . 127

5.2.2 Power-law Dependence and Organism Density . . . . . . . . 129 
5.2.3 A Physical-Reactive Continuum . . . . . . . . . . . . . . 131

5.3 Experimentally Testing Diffusion Limitation in Sediments . . . . . . . 132

5.3.1 Measuring the Effective Diffusion Coefficient in Porous Media 135

5.4 Measuring Diffusion Through NMR . . . . . . . . . . . . 136

5.5 Measuring Diffusion Through HPLC Methods . . . . . . . . . . . . 140

5.6 Final Thoughts . . . . . . . . . . . . . . . . 145

6 Parting Thoughts 147 


\section{Chapter 1}

\section{Introduction}

Inorganic carbon is taken up by photosynthetic organisms and transformed into organic carbon. This organic material, in turn, is oxidized back to carbon dioxide through respiration. It is both the most familiar, and dominant process occurring in what is known collectively as Earth's carbon cycle [1]. In order to model how this system operates we further divide the system into different sub-reservoirs of carbon based on both chemical properties and physical location. These models build in complexity with both our knowledge of the different carbon reservoirs and the elucidation of the flow mechanisms between them. This process could be important down to the nano-scale as there are at least thousands of distinct compounds found in naturally occurring reservoirs of organic carbon [2].

This work is motivated by the potentially simplifying presence of complexity in the carbon cycle. The sum of enough random events follow a normal distribution defined by a mean and a variance. In the same way, the networks of carbon reservoirs could be defined by a distribution of rate constants where the ensuing reservoir dynamics depend on the parameters of the distribution [3-5]. Some carbon reservoirs have already succumbed to this approach. Leaf litter decomposition on the forest floor, for example, can be well described by a lognormal rate constant distribution where the 
mean of the logarithm and the variance of the logarithm depend on temperature and precipitation [5]. The unifying presence of turnover time distributions is aesthetically pleasing, but must break down at some scale, to allow for external influences. For example, it is unreasonable to assume that organic carbon will decay with the same dynamics in Antarctica as it does on the floor of a rainforest.

The carbon cycle in the ocean seems especially prone to the inclusion of statistical ordering at some scale. The ocean is continually mixed with macroscale data supporting underlying order at the microscale. The most impressive of this data is the compilation of decay rate constants for organic mater in the ocean as a function of its age. The average decay rate changes linearly with the inverse age in a relationship that spans over ten orders of magnitude in time [6]. This ordering across many potential processes and time scales suggest that a statistical kinetics might be applicable to the marine carbon cycle. This thesis focuses on the marine carbon cycle, exploring ways in which considering organic reservoirs in terms of rate distributions can lead to insights into how the carbon cycle functions. The thesis is split into four main chapters. Chapter 1 focuses on the theory of rate distributions and contextualizes the carbon cycle in terms of age-structured models [7]. Chapters 2 and 3 take this framework and apply it to the oceanographic reservoir of dissolved organic carbon (DOC) in the ocean, seeking the turnover time distribution inside this reservoir. Chapter 4 looks at a different carbon system, organic carbon in sediments, exploring mechanisms to test whether physical heterogeneity is sufficient to generate the observed power-law slowdown in first order decay rate with time $[4,6]$.

Chapter 1 focuses on the relationship between bulk properties of the carbon cycle, and its underlying heterogeneity. One of the fundamental challenges in carbon cycle measurements is to take bulk observations like reservoir size and radiocarbon age and relate them to an estimate of the mean age and the carbon flux [1]. The most straightforward method is to assume that the age is equal to the turnover time of the reservoir 
and ignore internal heterogeneities [8]. However, the relationship between estimates of mean age and turnover time depend strongly on the turnover time distribution as any bulk reservoir can be hiding components with extremely fast turnover times alongside components with extremely slow turnovers [8-10]. In situations like this, the distribution of turnover times inside the reservoir is critical for determining bulk age and flux. We show that in some cases like a lognormal distribution, the deviation between the mean age and the mean turnover time can grow exponentially as a function of the heterogeneity of the system. Even assuming a single first order reservoir in steady state leads to a nonlinear transformation between the radiocarbon age and any estimate of the mean turnover time. We place parallel decay models $[4,5,11]$ in the context of age-structured differential equations [7], showing how the bulk reservoir properties are calculated as functions of the moments of the input rate distribution. When applied to the specific case of the lognormal distribution, reservoirs in steady state are also lognormal. The mean of the logarithm shifts, but not its variance. We suggest that this variance of the logarithm may be a constant quantity of the marine carbon cycle. Testing the connections between heterogeneous reservoirs and their bulk properties requires both the correct model system, and methods of probing the internal turnover time distribution.

Chapters 2 and 3 specialize on oceanic dissolved organic carbon (DOC) in the ocean, and probe the distribution of turnover times in the deep ocean reservoir of DOC. Chapter 2 focuses on what can be inferred from bulk DOC measurements and chapter 3 estimates the turnover time distribution directly. DOC in the ocean is a large, (600 Pg) [1], reservoir of carbon, whose estimated age of 6000 years in the deep ocean covers multiple ocean mixing times [8]. Radiocarbon data suggests that new DOC is produced in the surface ocean on top of this background recalcitrant fraction making the two fractions of approximately equal concentration in the surface [12]. More complex formulations consider DOC to be a combination of at least 
five distinct carbon groups with turnover times ranging from years to 40,000 years with a distinct separation between modern carbon and the other reservoirs $[13,14]$. Correlations between the radiocarbon age of DOC and its concentration in depth profiles unambiguously show the presence of radiocarbon modern material in the surface ocean [8]. What is less clear is if the deep ocean 'recalcitrant' fraction of the DOC reservoir is truly as isolated as currently believed [14]. If the deep ocean is being exposed to modern carbon through the dissolution of sinking particles from the surface ocean [15], then we might expect the dynamics of DOC in the deep ocean to be split between material with a fast turnover time, and material with a broad turnover time distribution skewed to longer turnover times. Unfortunately, the dominant signal from the DOC cycle in the ocean comes in the surface ocean [13], making it difficult to figure out the dynamics of the deep ocean reservoir.

As the dominant signal in the DOC system is from surface carbon, we look for higher order signals in the deep ocean for evidence of modern carbon. Bulk concentration data is suggestive of steady state inputs, as distinct increases in concentration occur in equatorial regions with increased particle fluxes [16]. Additionally, if DOC is in a state of parallel decay, the normalized variance in concentration data should decrease between the deep Atlantic and Pacific Oceans as deep water ages [17]. However, we find a distinct increase in the variance, consistent with the enhanced primary production in the equatorial Pacific and its associated particle flux [18]. The injection of radiocarbon into the carbon system from nuclear bomb testing provides an additional signal as to the steady state nature of the deep ocean [19]. Two component mixing in the DOC system leads to straight lines in 'Keeling Space', a plot of radiocarbon value vs. inverse concentration [10,20]. We find systematic deviations from linearity in Keeling Space which support the propagation of surface DOC all the way into the deep ocean.

A large modern component in the deep ocean DOC reservoir naturally explains 
the aging paradox of the Southern Ocean. As deep waters move from the Atlantic, through the Southern Ocean, and into the North Central Pacific DOC ages in a way that is inconsistent with an isolated deep DOC reservoir [21]. Although DOC in the deep Atlantic and Pacific is consistent with monotonic aging [12], the age deviation in the Southern Ocean is greater than five hundred years [21]. A steady state reservoir in the deep ocean resolves this paradox without the addition of special pre-aged labile sources. As deep water travels the ocean it contains a substantial quantity of carbon which equilibrates with surface particulate carbon. Surface carbon in the Southern Ocean is substantially older than the rest of the ocean due to the continual upwelling of radiocarbon depleted inorganic carbon [22]. Thus, the equilibrated modern carbon is artificially older, driving the apparent paradox. Employing a simple box mixing model we suggest that at least $10 \mu \mathrm{M}$ DOC $(\sim 30 \%)$ is equilibrating with the surface ocean and that the balance of material cycles at timescales substantially longer than 6000 years.

Chapter 3 focuses on measuring the distribution of radioisotopes inside the DOC reservoir in the laboratory. All of the available information in our system is hidden in the chemical subspecies inside the DOC. The perfect instrument would give us every compound, the amount of material, and an estimate of its age (radiocarbon age). Mass spectrometry provides a detailed map of the compounds in the extractable fraction [2], but their relative quantities are not defined. The isotopic values of the compounds are also unknown. Estimating the isotope distribution requires an instrument which can equate changes in mass with changes in isotope value. Fortunately, the radiocarbon value of DOC changes as a function of oxidation time under ultraviolet light [23]. Sacrificing compound resolution for isotope resolution, we use serial oxidation experiments along with a model for the kinetics of DOC oxidation [24] as a spectrometer for estimating the carbon isotope distribution inside single seawater samples containing DOC. These results agree with those presented above. We suggest 
that up to $30 \%$ of deep water DOC is radiocarbon modern. When we apply a turnover time distribution consistent with a first order steady state system, the balance of the material cycles with an apparent turnover time of 30,000 years. We also demonstrate a spread of at least $10 \%$ in the stable carbon isotope spectrum for DOC, suggesting that inputs external to oceanic plankton may be relevant to the cycling of DOC in the ocean.

Chapter 4 changes focus and returns to the prediction of a power law scaling between the effective degradation rate constant of organic material and its age. Two mathematical models exist which can replicate this behavior. Chemical heterogeneity poses that a broad suite of chemicals are produced and as the labile ones are degraded, the average of what is left behind decreases. Certain functional forms for this initial distribution will yield the proper scaling [3]. An alternate view is that the rate distribution is set not by chemical diversity, but by the diversity of the physical environment. A simplified version of an extracellular foraging model [25] then yields the correct scaling if the decay is diffusion limited [4]. This chapter starts with this Rothman-Forney model and applies it to organic matter loadings in ocean sediments. We find that in addition to the Middelburg scaling relation, physical heterogeneity is sufficient to explain the linear relationship between organic matter and the surface area of local sediment grains. The rest of the chapter focuses on methods to directly test diffusion limitation and to measure the diffusion coefficient of extracellular enzymes in sediments.

The carbon cycle is a complex network of carbon reservoirs and their transformations. As the system is highly complex, it makes sense to consider the turnover time distributions present within reservoirs below a certain scale. However, measuring that scale and the distributions is difficult. In the following, we start with the ideas of heterogeneous reservoirs, and end up uncovering a scale separation between two distinct chemical reservoirs in the deep ocean. Further work is required to uncover at 
what scales heterogeneity, rather than external physical properties (like particle flux) control the turnover time distributions of organic carbon in the ocean system. 


\section{Chapter 2}

\section{Statistical Kinetics of the Marine}

\section{Carbon Cycle}

A multitude of processes both large and small mediate the transfer of carbon between its organic and inorganic forms. This diversity produces a vast set of carbon reservoirs and interconnecting processes, the network of which is resolvable across many scales. In the ocean, these interactions range from physical large scale processes like airsea gas exchange, to the influence of microbial diversity on the decomposition of organic material at the microscale. Correctly estimating bulk properties of the carbon network, like net carbon flux, requires knowing both the geometry and flow across the network and how they change with observation scale. Different assumptions about smaller scales can lead to drastic over, or under estimates of flux when integrated over the entire network. Although the flux through the different reservoirs can be estimated from bulk properties like concentration and radiocarbon values, a vast array of sub reservoirs down to $>10^{6}$ unique chemicals exist in, for example, oceanic dissolved organic carbon. Whether this chemical diversity effects bulk properties depends strongly on its internal structure. We focus on the simplest possible scenario, that of a parallel relaxation system, and relate it to age structured models. We find that small changes in our assumptions regarding the dissolved organic carbon reservoir lead to $>30 \%$ fluctuations in the calculated carbon flux. By applying a lognormal turnover time distribution to oceanic DOC we fit bulk concentration data equally well to the currently established three component model, yet with three less parameters. It seems possible that organic carbon systems are complex enough to warrant a statistical averaging over the microscale. However, directly elucidating which network structures exist and their supported fluxes requires methods which link reservoir identification with methods capable of accurately measuring mass and age, so that we can treat smaller scale interactions with their correct mass and flux distributions. 


\section{$2.1 \quad$ Introduction}

Nearly $50 \mathrm{Pg}$ of inorganic carbon enters the oceanic food web through primary production, yet the fate of this material is less clear, and the subject of substantial biogeochemical research [18]. What is certain, is that a multitude of processes across many scales transport and modify this organic material as it travels through the ocean's carbon cycle. Unfortunately, which processes dominate is unclear, and wedded to the pertinent timescale of interest. For example, although a small portion of organic carbon flux from the surface ocean $(<1 \%)$ is annually buried in sediments [26], as an integrated quantity across thousands of years it can have a profound impact, affecting the global budget of oxygen [27]. However, if one is interested in the carbon cycle of the surface ocean at a yearly timescale, then perhaps these long-timescale processes are not relevant.

Matching the required resolution scale with the question is difficult because seemingly unimportant or small scale processes can have large impacts for the system as a whole. If we seek to understand global scale carbon storage questions, then a statistical understanding of the carbon cycle is sufficient, but which portions can be averaged over and how do we perform the averaging? Under what conditions, if any, can we express respiration in the ocean as a statistical turnover time distribution, and what are the master variables? If such a statistical kinetics were possible, it would allow a predictive capability which is difficult to attain with models of increasing complexity.

The standard modeling approach for the carbon cycle is to recognize that it exists as a set of chemical and spatial reservoirs which form a network on which carbon flows from one box to another $[1,28,29]$. The simplest such representation reduces the carbon cycle to two reservoirs; organic and inorganic carbon (Fig. 2-1). If we fix primary production, $p$, and assume that the organic reservoir is a first order system with rate constant $k$ then we can write the organic carbon concentration, $C$, as a first 


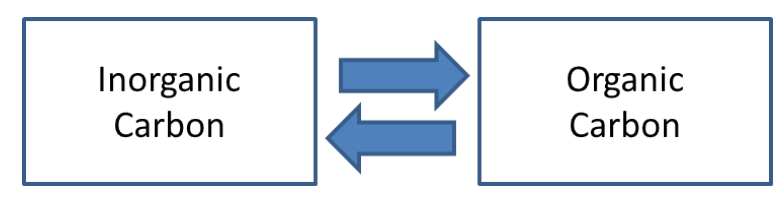

Figure 2-1: The simplest representation of the carbon cycle consists of two interconnected reservoirs, (Inorganic Carbon and Organic Carbon).

order differential equation. Mass balance yields

$$
\frac{d C}{d t}=-k C+p
$$

which reduces to

$$
p=k C
$$

in steady state. Measuring fluxes directly is extremely difficult, forcing flow to be inferred from bulk properties of the organic reservoir $[8,13,16,30,31]$. The most common way to infer fluxes is to use spatial gradients to calculate changes between regions, and then assume that net and total fluxes can be equated [13,16,31]. The potential danger of such an approach is seen in this simple model. Even a very small reservoir can support a very large steady state flux if the rate constant is high enough (or the turnover time low enough).

Nothing in this, simplest, manifestation of the carbon cycle is specific to the ocean. Also, as it integrates over the entire carbon cycle, there are natural questions as to the measurement of the reservoir sizes, and the assumption that the transfer of carbon can be governed by a single rate constant. To specialize to the ocean and add some additional realism we could split the organic fraction into sub-reservoirs. In the surface ocean one might envision three distinct species of organic material; biomass, particulate organic carbon (POC), and dissolved organic carbon (DOC) (Fig. 2-2) [1]. Biomass is an excellent example of a small $(<2 \mathrm{PgC})$ reservoir whose yearly fluxes $(\sim 50 \mathrm{PgC})$ are extremely large [32]. We can extend this model spatially 


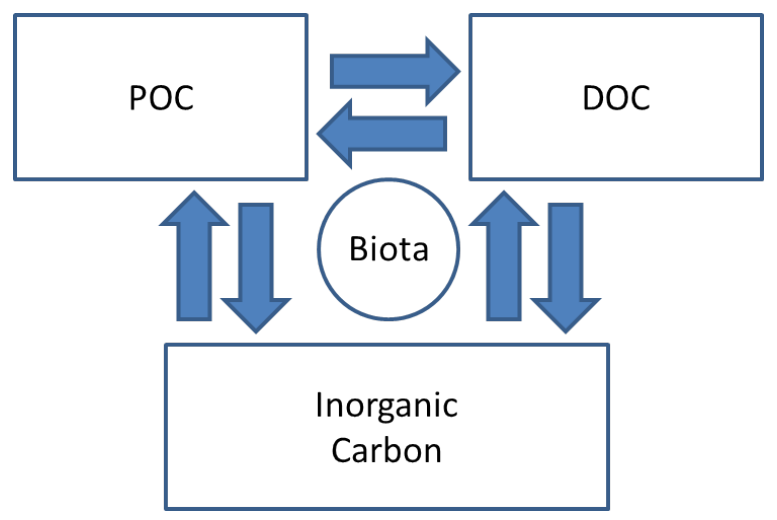

Figure 2-2: The organic carbon cycle is better represented in the ocean by splitting the organic fraction into particulate (POC) and dissolved (DOC) phases and by recognizing that the carbon transfer is biologically mediated.

in a representative depth profile where the relative reservoir sizes and fluxes are now depth dependant (Fig. 2-3) [8,33].

An understanding of the ocean carbon cycle as presented in figure 2-3 requires two things. First, a knowledge of the local carbon system (internal structure and dynamics) and second a knowledge of how the system equilibrates under differing external environments. These environments, based on interpreting figure $2-3$ might be warm light rich surface ocean, the dark cold nutrient rich deep ocean, and the minearal-solid phase rich ocean sediments. The internal system consists of the species of organic material (boxes), the transfer paths between them (network geometry), and their interconversion rates. These ideas are similar to the solution of an ordinary differential equation through the use of homogeneous (internal), and particular (external) solutions.

Focusing on the internal dynamics of the ocean carbon cycle returns us to figure 2-2 and the three interconnected species of organic carbon. Does this diagram fully describe the system? It depends. POC and DOC are clearly not single compounds and biomass not a single species $[2,34,35]$. In the dissolved reservoir alone, recent work using Fourier transform mass spectrometry methods has uncovered at least 


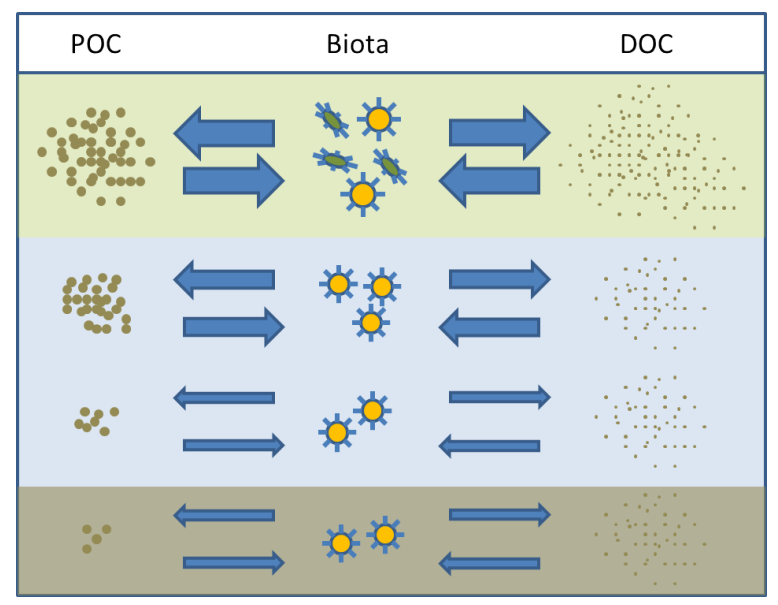

Figure 2-3: The biologically mediated transfer of organic material between phases is extended spatially as a function of depth in the ocean. Photosynthetic primary production exists solely in the surface ocean leading to the majority of organic material existing here. The decreasing reservoirs of organic carbon with depth are due to the continued degradation, with far less replacement.

millions of individual compounds. Measuring DOC and POC in the ocean is at least conceptually straightforward as POC can be mechanically filtered to separate the two components [1]. However, if chemical composition is more important than physical phase, how do we approach the complexity? The number of potentially important connections scales as the square of the relevant number of reservoirs of carbon. This leaves us with nearly $10^{12}$ possible transformations to sift through, before we add the additional complexities of local environment and ecosystem.

Reducing the carbon cycle first to the ocean, and then to the water-column leaves us with tremendous complexity in the mass distribution among the chemical species. Modeling this system requires that simplifications be made which make the problem solvable either analytically or through simulation. We assume here that the complexity is found in the chemical species, and that the transfer kinetics are simple and expressible by first order kinetics. Naturally, both things must be wrong as the oxidation of organic matter depends on the chemical species, its local physical-chemical environment, and the suite of organisms which are present. 
A perfect model would include all of the chemical species, and follow their creation, transformation, and oxidation explicitly as a function of time and space. However, not only do we not have the computing power to accomplish such a task, we do not have the appropriate knowledge of the individual species and their transfer kinetics. The ideal situation is that the details of such a system can be completely ignored, that the underlying complexity can be isolated from what happens at the large scale. Under this situation, following parts of the carbon cycle which are straightforward to measure (DOC, POC, sediment carbon) is sufficient.

Finding the correct averaging scale is difficult, and the normal progression is to start with the largest scale possible and work down as time, need, and resources permit. We seek an intermediate formulation which includes the micro-scale complexity, but in a mathematically tractable fashion. We seek a fundamental distribution for the network below a cutoff scale which allows us to understand the system in terms of a differential equation for that distribution. When calculating the flux through the carbon network by employing first order models of increasing complexity, we assume that the resolution of each additional scale will only slightly modify the results attained at the previous scale. If the micro-scale network structure is vastly different than the macro-scale, our understanding of the network response could be equally flawed. If the system is complex enough, perhaps the micro-scale complexity can be averaged over and thought about statistically in terms of a rate distribution.

\section{$2.2 \quad$ Age-Structured Models}

Let us assume that the internal dynamics of the carbon cycle can be expressed as a network governed by first-order decay. In this case, we might represent the network as a carbon reservoir where the amount of material, $M(a, k, t)$, depends on its decay constant, $k$, age, $a$, and the amount of time, $t$, the system has been allowed to evolve. 
Physically, we have exchanged the details of each chemical with a rate constant and a mass. This system is expressed by the age-structured differential equation

$$
\frac{\partial M(a, k, t)}{\partial t}=-\frac{\partial M(a, k, t)}{\partial a}-k M(a, k, t)+I(a, k, t)+\int_{0}^{\infty} k^{\prime} f\left(k, k^{\prime}\right) M\left(a, k^{\prime}, t\right) d k^{\prime} .
$$

The change in the mass distribution is dependent upon age advection (term 1), first order decay (term 2), external inputs (term 3), and the transfer of carbon between reservoirs (term 4). The function $f\left(k, k^{\prime}\right)$ sets the fraction of decay products which travel from reservoir, $k^{\prime}$, to reservoir $k$ upon decay. Equations of this form are often used in the fields of epidemiology and population dynamics [36, 37].

If the organic carbon network can be isolated from external inputs, $I(a, k, t)$, and internal transfers within the system can be ignored equation (2.3) can be reductd to the continuous formulation of the multi-G model [11]. Conceptually, we are reducing a network of carbon species into a set of non-interacting carbon reservoirs in which the bulk behavoir is a sum of non-interacting parts (see figure 2-4). Setting external inputs and transfers to zero reduces equation (2.3) to

$$
\frac{\partial M(a, k, t)}{\partial t}=-\frac{\partial M(a, k, t)}{\partial a}-k M(a, k, t)
$$

Integrating over the age then yields

$$
\frac{d M(k, t)}{d t}=-k M(k, t)
$$

If there are no inputs to the system, then the time evolution can be described completely in terms of the mass distribution $M_{0}(k)$ at $t=0$, as

$$
M(k, t)=M_{0}(k) e^{-k t}
$$




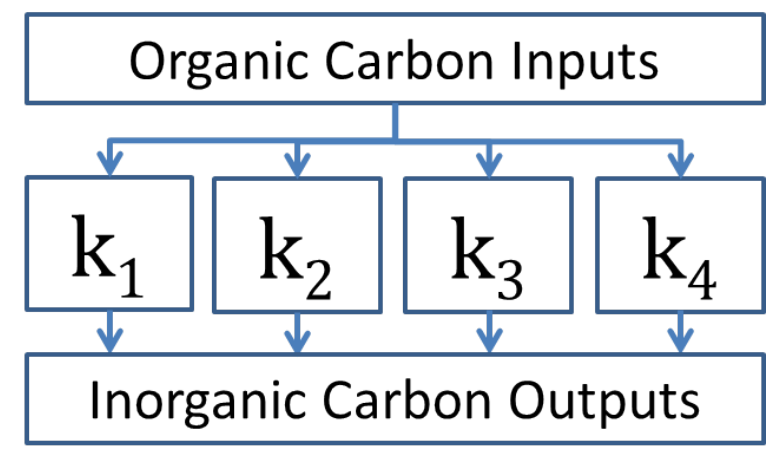

Figure 2-4: We treat the carbon cycle as being governed by a set of reservoirs ( $\mathrm{k}$ boxes) which cycle in parallel. If we can link the reservoirs through a distribution then we can decrease the complexity of the system dramatically.

The mass evolution of the system, $M(t)$, is then

$$
M(t)=\int_{0}^{\infty} M_{0}(k) e^{-k t} d k
$$

Equation (2.7) is the impulse response of our simplified carbon network.

Returning to equation (2.4) we can solve for the age distribution of a steady state reservoir with a constant input flux, $F$, of zero age. The constant flux boundary condition requires that

$$
F=\int_{0}^{\infty} M(a=0, k, t) d k
$$

Integrating time out of equation (2.4) leaves

$$
\frac{d M(a, k)}{d a}=-k M(a, k)
$$

which can be solved in terms of the initial distribution,

$$
M(a, k)=M_{0}(k) e^{-k a} .
$$


Integrating out the rate constant yields the age distribution

$$
M(a)=\int M_{0}(k) e^{-k a} d k
$$

which is identical in form to the 'impulse response' of our system $M(t)$ with no external inputs.

Equation (2.3) is a $k$-dependant version of the von Foerester equation $[7,36]$

$$
\frac{\partial N}{\partial t}=-\frac{\partial N}{\partial a}-K(a) N
$$

with the boundary condition for $a$ that

$$
N(0, t)=\int_{0}^{\infty} b(a) N d a
$$

Here, $N$, is the number of individuals, $K(a)$ is the age dependant death rate, and $b(a)$ is the age dependant production or birth rate. Our formulation removes the complexity of the age dependant birth and death rates, and replaces it with the complexity of a distribution of underlying rates. The conceptual difference between the systems comes from the age term. In the population setting, individuals of zero age are created as a function of the total population size, whereas in the carbon system described here production is independent of the current reservoir size.

\subsubsection{Equating Reservoir Properties with Age Structure}

The equivalence of the free mass decay with the steady state age distribution allows us to equate bulk properties of the steady state reservoir to the rate distribution of the fluxes, $F(k)$, into the system and the moments of the distribution. The normalized distribution $\rho_{0}(k)$,

$$
\int_{0}^{\infty} \rho_{0}(k) d k=1
$$


is then related to $F(k)$ through the constant mass flux into the system, $F$, where

$$
F(k)=F \rho_{0}(k) .
$$

The mass $M$, flux $F$, average age $\langle a\rangle$, and turnover time $\tau$ can all be related to the $n^{\text {th }}$ integer moments, $n$, of the normalized flux distribution

$$
m_{n}=\int k^{n} \rho_{0}(k) d k \text {. }
$$

The mass of the reservoir can be expressed as the integral over the entire distribution,

$$
M=\iint M(a, k) d a d k,
$$

which can be expanded and solved,

$$
M=\iint F(k) e^{-k a} d a d k=F \int \frac{1}{k} \rho_{0}(k) d k=F m_{-1},
$$

in terms of the flux and the negative first moment. As we have assumed first order kinetics, the expression for the flux

$$
F=\iint k M(a, k) d a d k
$$

is closely related to the integral expression for the mass. This too can be expanded to find the trivial relation that

$$
F=\iint k F(k) e^{-k a} d a d k=F \int \rho_{0}(k) d k=F m_{0} .
$$

The expressions for the mass and flux are identical, except that the mass expression contains the negative first moment, while the flux contains the zeroth. The mean age 
of the reservoir is calculated from the entire normalized distribution,

$$
\langle a\rangle=\frac{1}{M} \iint a M(a, k) d a d k,
$$

which can be expanded in the above fashion to yield

$$
\langle a\rangle=\frac{1}{M} \iint a F(k) e^{-k a} d a d k=\frac{F}{M} \int \frac{1}{k^{2}} \rho_{0}(k) d k=\frac{m_{-2}}{m_{-1}},
$$

or the ratio between the negative first and negative second moments of the flux distribution.

Calculating the turnover time requires a thoughtful consideration as to what the turnover time is. In general, the turnover time is the time required for a mass equivalent to the reservoir mass to flow through the reservoir [38]. This is equivalent to the mass to flux ratio. In the current formulation we can find the turnover time by rearranging equation 2.18. This is expressed as

$$
\tau=\frac{M}{F}=m_{-1}
$$

where the turnover time is equal to the negative first moment of the flux distribution.

Before moving on it is important to note that although the mean age and the turnover time both have units of age, they are rarely equivalent. They only equate when the negative second moment is equal to the square of the first as

$$
\frac{\tau}{\langle a\rangle_{0}}=\frac{m_{-2}}{m_{-1}^{2}}
$$

This ratio is only equal to unity when there exists a single decay rate in the system. This is the only situation where the mean age and turnover time are equivalent [38]. 


\subsubsection{The Lognormal Rate Distribution}

Laplace transform formulations like equation (2.7) are useful if the initial rate distribution, $M_{0}(k)$, or its functional form can be understood in terms of a small set of

physical variables. Fortunately, there is evidence for master distributions in at least two environments relevant to the carbon cycle. Recent work on terrestrial systems suggests that leaf litter oxidation may be well described by equation (2.7) where the flux distribution is Lognormal,

$$
F(k ; \mu, \sigma)=F \frac{1}{k \sigma \sqrt{2 \pi}} e^{\frac{-(\ln k-\mu)^{2}}{2 \sigma^{2}}}
$$

( $F$ is a constant which sets the flux) and arises due to applying the central limit theorem to the heterogeneous chemical, biological, and physical environment of the forest floor [5]. The parameters $\mu$, and $\sigma$ were found to vary with the climate relevant variables of temperature and precipitation [5].

If the input rate constant distribution is lognormal, then the internal distribution of the bulk properties discussed above is also lognormal. This comes from the shape invariance of the lognormal when multiplied by $k^{n}$ where $k>0$ and $n$ is a real number. We can demonstrate this by multiplying $k^{n}$ by both sides of equation (2.25),

$$
k^{n} F(k ; \mu, \sigma)=F \frac{1}{k \sigma \sqrt{2 \pi}} k^{n} \exp \left(\frac{-(\ln k-\mu)^{2}}{2 \sigma^{2}}\right),
$$

and re-working the right hand side. With our constraints on both $k$ and $n$

$$
k^{n}=e^{\ln k^{n}}
$$

which allows us to include $k^{n}$ in the exponential term,

$$
k^{n} F(k ; \mu, \sigma)=F \frac{1}{k \sigma \sqrt{2 \pi}} \exp \left(\frac{-(\ln k-\mu)^{2}}{2 \sigma^{2}}+n \ln k\right) .
$$


If we complete the square in the exponential and separate out the constant we get

$$
k^{n} F(k ; \mu, \sigma)=e^{n \mu+n^{2} \frac{\sigma^{2}}{2}} F \frac{1}{k \sigma \sqrt{2 \pi}} e^{\left(\frac{-\left(\ln k-\left(\mu+n \sigma^{2}\right)\right)^{2}}{2 \sigma^{2}}\right)} .
$$

which is the same as

$$
k^{n} F(k ; \mu, \sigma)=e^{n \mu+n^{2} \frac{\sigma^{2}}{2}} F\left(k ; \mu+n \sigma^{2}, \sigma\right) .
$$

This shows that multiplying the lognormal by $k^{n}$ yields a shifted lognormal distribu-

tion rescaled by the constant $e^{n \mu+n^{2} \frac{\sigma^{2}}{2}}$. Integrating both sides demonstrates that the re-scaling constant generates the moments of the lognormal where

$$
\left\langle k^{n}\right\rangle=e^{n \mu+\frac{n^{2} \sigma^{2}}{2}} .
$$

\subsubsection{Application to Dissolved Organic Carbon}

Dissolved organic carbon (DOC) is the dominant reservoir of reduced carbon in the ocean and is heterogeneous in nature, with at least $10^{6}$ unique chemicals [2]. Unlike sediments and the terrestrial environment which are analyzed in terms of smooth initial rate distributions $[5,11,39,40]$, oceanic DOC is studied based on discrete components of different turnover time $[8,13,16]$. Depending on the study, there are believed to be between two and five compound groups with differing decay constants. What chemically or physically distinguishes these groups is a matter of conjecture [16]. What is widely accepted however, is that the vast majority of DOC in the ocean comes originally from planktonic production in the surface ocean [12]. This single source makes the premise of a chemically based rate distribution, such as can be found in leaf litter, to be quite appealing.

If we assume, for the moment, that DOC inputs to the ocean are lognormally 
distributed, equation (2.25), it becomes possible to constrain the entire system with three parameters; normalization, $\mu$, and $\sigma$. Thus, three measurements are required. For DOC, we have estimates for the total mass $(M=600 \mathrm{Pg})[30]$ the total flux $(F=50 \mathrm{Pg} / \mathrm{yr})[18]$, and an estimate for the average age of the organic reservoir $(\langle a\rangle=6000$ years $)[8]$.

We seek an expression for both the turnover time $(\tau=M / F)$ and the mean age $(\langle a\rangle)$ in terms of the parameters of the lognormal $(\mu$ and $\sigma)$. However, unlike leaf litter or sediments, the ocean is a bulk reservoir in steady state whose bulk properties are defined by the equations of section 2.2.1 along with the expression for the moments of the lognormal distribution,

$$
\left\langle k^{n}\right\rangle=e^{n \mu+\frac{n^{2} \sigma^{2}}{2}}
$$

derived above. The mean age is the ratio of the second and first negative moments of the lognormal,

$$
\langle a\rangle=e^{-\mu+\frac{3 \sigma^{2}}{2}},
$$

while the total mass to flux ratio equals the negative first moment

$$
\tau=\frac{M}{F}=e^{-\mu+\frac{\sigma^{2}}{2}}
$$

Combining equations 2.33 and 2.34 we can solve for both the log mean and variance as

$$
\mu=\ln \left(\sqrt{\frac{\langle a\rangle}{\tau^{3}}}\right)
$$

and

$$
\sigma^{2}=\ln \left(\frac{\langle a\rangle}{\tau}\right)
$$

Substituting the total mass $(M=600 \mathrm{Pg})$, the total flux $(F=50 \mathrm{Pg} / \mathrm{yr})$, and an 
estimate for the average age of the organic reservoir $(\langle a\rangle=6000$ years $)$ into these equations we estimate that $\sigma=2.5$ and $\mu=.75$ for the input distribution.

We can estimate the parameters of the lognormal distribution independently by fitting to DOC concentration data in the deep ocean [16]. As deep ocean water moves from the north Atlantic ocean to the North Pacific, it ages and its DOC concentration decreases [12]. Estimating the water age from radiocarbon [22] and equating it with the DOC concentration data allows us to get an estimate for DOC concentration as a function of age relative to the deep north Atlantic (Figure 2-5). Fitting this data to a lognormal decay distribution suggests that $\sigma=2.6$ and $\mu=-10.6$ for the input distribution of this data. Clearly, the estimate for $\mu$ from the deep water data is different than that for the bulk estimate above. However, the log variance is extremely close. This is to be expected, as the initial condition for the deep water decay is closest to a steady state reservoir. The deep decay fit allows us to estimate the steady state distribution, while the bulk estimate is for the input distribution.

The initial condition for this data set is subsiding surface water from the north Atlantic. Presumably, this surface water has seen many mass turnovers of DOC as it is the location of DOC production $[18,31]$. Thus, the initial condition is closer to the steady state reservoir than it is to the impulse response. If the reservoir is in steady state, we can write the rate distribution

$$
M(k)=\int_{0}^{\infty} F(k) e^{-k t} d t=F k^{-1} \rho_{0}(k) .
$$

If, as we have assumed here, the flux distribution is lognormal, $\rho_{0}(k ; \mu, \sigma)$, then we can use equation (2.30) to show that

$$
M(k)=e^{\frac{\sigma^{2}}{2}-\mu} \rho_{0}\left(k ; \mu_{s s}, \sigma_{s s}\right)
$$




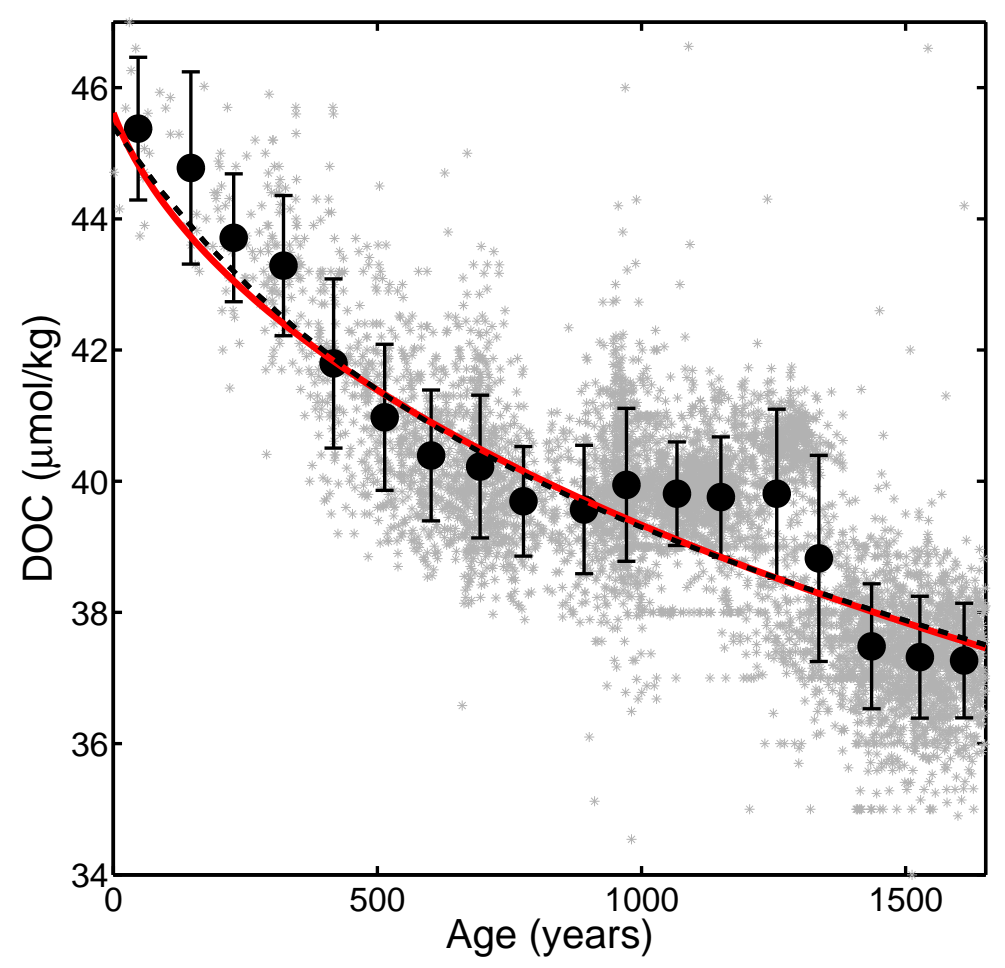

Figure 2-5: A lognormal, parallel decay model (red solid line) fit to DOC concentration data as a function of water age for data below 2000 meter depth [16, 16, 22]. The entire data set (gray stars) is overlayed by estimates for the mean and standard deviation for 20 evenly spaced regions in time. The best fit for two exponential components (black dashed line) is almost identical yet contains an extra parameter. The lognormal fit has $\sigma=2.6$ and $\mu=-10.6$ for the lognormal distribution of rates. 
which is also lognormal where

$$
\mu_{s s}=\mu-\sigma^{2} \text { and } \sigma_{s s}=\sigma
$$

This is a special situation for the lognormal, which as shown earlier retains its shape under multiplication by $k^{n}$. Using these equations, our estimate for the initial parameters of the lognormal is $\sigma=2.6$ and $\mu=-5.5$ for the input distribution. This is substantially more reasonable. The additional deviation between the log mean estimates could be due either an incorrect estimate for the initial zero time, the fact that the surface ocean is not a truly equilibrated reservoir, or to a lack of true separation between the surface and deep oceans.

The data fit in figure 2-5 contains thousands of data points taken from all of the major oceans below 2000 meters [16]. The concentration data is paired with radiocarbon ages interpolated from global radiocarbon data for dissolved inorganic carbon [22]. To capture the aging of the deep ocean, this data was shifted so that the age is the age relative to the source of the deep DOC, the North Atlantic. This process treats all data as having equal weight independent of sample density across basins. Also, in treating the radiocarbon age as indicative of the actual age, we ignore any and all mixing processes. It is clearly important to integrate distribution models like this one and others [11] into global ocean biogeochemical models [13], in order to more accurately compare their efficacy. The use of the lognormal distribution, however, has a few advantages, both conceptually and practically. From a practical standpoint, although the bulk behavior of DOC can be replicated equally well by either the lognormal distribution or a more traditional multi-exponential fit, the multiexponential has an additional degree of freedom. If we can explain the bulk behavior of a reservoir with less parameters, then we have a better chance of understanding the system as a whole. Additionally, the functional form of the distribution is directly 
related to the internal structure of the reservoir.

\section{$2.3 \quad$ Heterogeneity and Bulk Measurements}

Connecting a measurement of bulk carbon concentration with a flux requires the measurement of a timescale. Above, we assumed that deep ocean DOC was isolated from sources of carbon and connected changes in spatial location with changes in time. Direct estimates of the average age of carbon can be made through radiocarbon estimates. Radiocarbon $\left({ }^{14} \mathrm{C}\right.$ a radioactive isotope of carbon) is continually made in the atmosphere and is approximately at a constant concentration. When this carbon is incorporated into organic material, the radiocarbon slowly decays away, leaving a

fingerprint as to the age since production. At least two independent issues must be overcome to equate a bulk radiocarbon age estimate with an estimate of the mean turnover time.

The most straightforward method of equating a mean age estimate, $\langle a\rangle$, with a turnover time is to realize that decay constant has units of inverse age, and equate the age with the turnover time, $\tau$. If a reservoir is made up of a single, first order component with rate constant $k$, then this expression is correct. Letting

$$
M(a, k)=F e^{-k_{1} a}
$$

and integrating $a M(a, k)$ yields

$$
\langle a\rangle=\frac{1}{k_{1}}
$$

or what our intuition suggested [38]. If the input distribution is more complicated, then the relation between the turnover time and the average age is also nontrivial. Equating mean age and mean turnover time is akin to equating the mass to flux ratio with mean age. If the input distribution is lognormally distributed as above, then 
the turnover time is exponentially dependent on $\sigma$ where the ratio of the mean age and turnover time

$$
\frac{\langle a\rangle}{M / F}=e^{\sigma^{2}}
$$

When connecting bulk age measurements to turnover times and fluxes, it is thus highly important to carefully consider the underlying age structure of the reservoir. This is especially important considering the fact that our measurement of age is based on the exponential decay of an isotopic species.

\subsubsection{Equating Radiocarbon Age with Flux in a First Order Reservoir}

Measuring the age distribution in natural, well mixed reservoirs and connecting it to a bulk flux is a multi-step process as the main tool connecting organic carbon to its age comes from environmental radiocarbon. The ratio of radiocarbon to stable carbon, $R$, stays relatively constant in Earth's atmosphere [1]. When it is reduced to organic carbon, it acts as a time piece where the concentration of radiocarbon in a sample decays exponentially with time,

$$
R(t)=R_{0} e^{-\lambda t}
$$

where $R_{0}$ is the initial radiocarbon concentration and $\lambda=\frac{1}{8267} \mathrm{yr}^{-1}$ is the radiocarbon decay constant [1]. The radiocarbon age, $A_{r}$, is then

$$
A_{r}=\frac{-\ln R / R_{0}}{\lambda}
$$

Current isotopic estimates of carbon flux through DOC assume that the radiocarbon age of a sample equals its mean age, $A_{r}=\langle a\rangle$ [8]. However, this is only true if all material in the reservoir has a single age [8]. In general, the mean age of a sample is 
related to its radiocarbon age through its normalized age distribution, $\rho_{0}(a)$, where the measured isotopic ratio

$$
R=\int_{0}^{\infty} \rho_{0}(a) R_{0} e^{-\lambda a} d a
$$

This can be rephrased in terms of the radiocarbon age

$$
A_{r}=\frac{-\ln \left(\int_{0}^{\infty} \rho_{0}(a) e^{-\lambda a} d a\right)}{\lambda}
$$

where $A_{r}=\langle a\rangle$ only when $\rho_{0}(a)=\delta\left(a^{\prime}-a\right)$. If the reservoir is in first order steady state with a single rate constant $k_{0}$ then $\langle a\rangle=1 / k_{0}$ and the relationship between the mean age and the radiocarbon age

$$
\langle a\rangle=\frac{e^{\lambda A_{r}}-1}{\lambda}
$$

which is nonlinear because the time resolution of radiocarbon decreases with age. Using a radiocarbon age for DOC of 6000 years [8] suggests a mean age closer to 9000 years and a decrease in the projected carbon flux by $30 \%$.

Researchers have long recognized that deep water DOC contains a range of radiocarbon values and masses. Mostly, this is expressed by recognizing that any deep water radiocarbon value could be a sum of two different components, spanning a range of possible masses and radiocarbon values [8-10]. In this case, the possible bulk turnover times span a tremendous range. In the extreme case DOC contains a modern component which has a measured turnover time of zero and a radiocarbon dead component which could have infinite turnover time. Clearly, the underlying distribution in the DOC reservoir has large implications to the bulk behavior. Depending on the assumptions made about the internal dynamics of DOC, we can attempt to correct for the heterogeneity. 


\subsubsection{Measuring the Turnover Time Distribution}

Heterogeneity hidden in bulk reservoirs of organic carbon may or may not matter to the carbon cycle as a whole. If external factors, like physical protection in sediments [40], regulate a reservoir then its internal complexities might have little global effect. In order to determine how much chemical complexity in a carbon reservoir is related to complexity in the dynamics, we must find a way of measuring the turnover time distribution of the components. Understanding the turnover time distribution of DOC and other heterogeneous reservoirs in the carbon cycle allows us to determine at what scales a statistical, like the lognormal model presented above, rather than a multiple reservoir model best fits the local dynamics. It is clear that some forms of carbon show a distinct separation in their behavior when compared with other forms. The inorganic, gaseous form of carbon operates on a different timescale and with differing dynamics than organic material in soils. In this case the idea of two distinct, yet connected reservoirs makes tremendous sense. DOC in the ocean is heterogeneous enough that although distinct compounds exist, the entire reservoir could be interconnected and have behavior expressible by a single age and rate distribution. On the other hand, the chemical complexity could be hiding a simpler dynamics consisting of a handful of reservoirs which operate separately.

Reservoirs which should be split into multiple components should be determinable by observing the distribution of turnover times, $P(\tau)$, inside the reservoir. Here, we have let the turnover time be a function of the rate constant, $\tau(k)$. In general, for a reservoir governed by first order decay, the age distribution is strictly monotonic [38]. This is not true for the turnover time distribution. In reservoirs with discrete dynamics, we expect the turnover time distribution to be multi-modal where each mode or peak represents a distinct group of material cycling with distinct dynamics, (decay rates $k$ ). For example, DOC in the surface ocean is believed to consist of two reservoirs, one labile and one of refractory carbon. In this case, we would expect that 


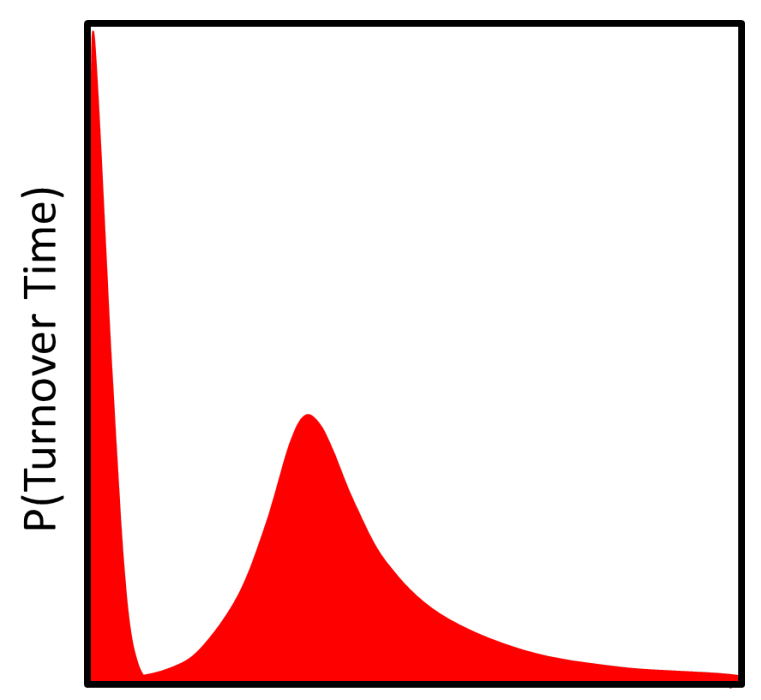

Turnover Time

Figure 2-6: A schematic of what we might expect the turnover time distribution of DOC to look like in the surface ocean if we measured the turnover time and total mass of all chemicals inside the DOC in the surface ocean. The labile and recalcitrant fractions [8] of the surface ocean are seen by the peak at short turnover times with low variance, and the peak at long turnover times with high variance.

the turnover time distribution for surface ocean DOC is distinctly bimodal with a sharp peak at near one year and a broad peak near 6000 years (See fig. 2-6).

Earlier, we argued that the carbon cycle, or parts of it, could be split into internal and external dynamics. If we are interested in measuring and understanding the turnover time distribution of a reservoir like DOC, we can attempt to measure it by analyzing fluctuations in bulk properties $[8-10,12]$, or by trying to directly measure the internal distribution using laboratory gradients [23,41,42]. Bulk fluctuations in the environment are problematic because there are many external variables which make the transformation from a spatial variable to a time variable problematic (see above discussion on DOC concentration). The advantage of temporal-spatial measurements, is that decay variability which is not based on chemical structure can be inferred [5].

When a single sample is being used to determine the turnover time distribution, 
the possible available information is relegated to the diversity in chemical species and their isotopic composition $\left({ }^{14} \mathrm{C}\right.$ for age and ${ }^{13} \mathrm{C}$ for potential origin). Thus, the perfect instrument would measure the entire chemical spectrum, and the concentration of each compound. Unfortunately, current methods of measuring organic carbon are either very good at quantifying the mass, (ultraviolet oxidation, high temperature catalyzed oxidation), or cataloging a subset of the chemical species, (extraction coupled with mass spectrometry). The fundamental dilemma is one of sensitivity versus specificity. If you want to access a specific molecule, you sacrifice resolution as to the amount. Estimating the distribution requires an instrument which trades off molecular understanding for the ability to connect an amount to an isotopic value.

Isotope spectrometric methods which separate chemicals along a gradient allow for the direct estimation of the isotope distribution inside a reservoir at the cost of direct knowledge of the chemical species. The most well developed of these methods is based on oxidation across an imposed thermal gradient [41]. This machine operates on the basis that different organic compounds are oxidized to carbon dioxide at different temperatures. In this system, one measures the isotope ratio as a function of temperature, $R(T)$, and carbon mass oxidized as a function of temperature, $M(T)$. What we desire is an estimate of the mean age distribution, $P(\langle a\rangle)$, which we estimate from the isotope distribution, $P(R)$. Extracting the age distribution requires eliminating the gradient variable, in this case temperature, through a sifting formula

$$
P(R) \approx \frac{\int_{0}^{\infty} M(T) \delta(R-R(T)) d T}{\int_{0}^{\infty} M(T) d T}
$$

where $P(a)$ and $P(\langle a\rangle)$ can be directly determined from $P(R)$. This simple estimation procedure can be done with any gradient over which $R$ fluctuates, be it a chemical gradient in the laboratory, or a physical gradient in the ocean. Additional information can be had if a deconvolution theory is available for the gradient series. The best 
example of a deconvolution instrument is nuclear magnetic resonance, NMR, where the bulk signal is related to the shift spectrum through a Fourier transform. In this case the bulk signal is understood to be a sum of independently oscillating nuclei. Estimating the isotopic distribution using an oxidating timeseries can be enhanced using the underlaying kinetic behavior of this system. Recent work on the oxidation of organic carbon by ultraviolet radiation in the laboratory [43] suggests that it is a parallel oxidation process where chemicals with different radiocarbon ages have different degradation rate constants when exposed to ultraviolet light.

The oxidation timeseries, $g(t)$, can be expressed as an integral of the rate distribution times the kinetic behavior, or

$$
g(t)=\int P(k) f(k, t) d k
$$

A parallel first order system can be expressed as a Laplace transform where

$$
g(t)=\int P(k) e^{-k t} d k
$$

If the isotopic behavior is also known then it is approximately expressed

$$
R(t)=\frac{1}{g(t)} \int R(k) P(k) e^{-k t} d k
$$

Deconvolving out $P(k)$ and $R(k)$ using theory surrounding the inverse Laplace transform allows us to estimate an age distribution from any kinetic decay experiment where

$$
P(R)=\int P(k) \delta(R-R(k)) d k
$$

and $R$ is equated to age as before. In this way, an entire class of instruments based on kinetic deconvolution could be made to directly estimate the isotopic distrubution of organic carbon reservoirs in the water, soil and rock records. 


\section{$2.4 \quad$ Final Thoughts}

Technology designed to categorize the constituents of biogeochemical systems have revolutionized our understanding of biogeochemically relevant processes on the microscale $[44,45]$. The omics revolution combined with advancement of mass spectral techniques has allowed us to unravel both the microbial and chemical diversity of carbon cycling. It does not seem far fetched that we will soon be able to take a bottle of seawater and get a full profile as to the genetic diversity, metabolic diversity, and chemical diversity in a single sample. However, connecting these maps of what is there to the dynamics of the carbon cycle is unclear. How much of this complexity is important and present as we move up to larger scale questions?

The simplest thing to do from a modeling perspective is to ignore the small scale complexity and its associated network. Here, we assume that a direct measurement of the spatial fluctuations of the property of interest (DOC for example) is sufficient to understand its dynamics. However, as we showed above, depending on what assumptions are made, different bulk properties and their spatial variability can be associated with vastly different bulk fluxes. One approach is to continually increase the complexity of our models until they are an in-silica representation of reality. Unfortunately, the inherent complexity of the system makes this approach difficult.

We propose that the system may be complicated enough to warrant a thermodynamic understanding below a certain scale. In this case, the details are not important, only that the system will approximate the same turnover time distribution under some set of master variables. The challenge is to develop an understanding of what the correct statistical models are, and find ways of estimating across what spatial-temporal scales they can be used. In some areas of the carbon cycle, (leaf litter decay, oxidation in sediments), these methods have already found a place in simplifying how we think about carbon cycling. In these cases, an underlying rate distribution (lognormal [5], log-uniform [40]) have combined with a small set of master variables (clay surface 
area, temperature, precipitation) to unify complex decay under a differential equation for a probability distribution. In order to move forward we must see an increase both in our theoretical understanding of these age-structured models, and in methods designed to measure the proper distributions and their applicable scale. 


\section{Chapter 3}

\section{Is Dissolved Organic Carbon in the Deep Ocean in a Dynamic Steady}

\section{State?}

Marine dissolved organic carbon (DOC) is a large (660 Pg C) reactive carbon reservoir that flows through the oceanic microbial food web, and stores reduced carbon on both short and long timescales. Two main methods exist to uncover the bulk cycling of DOC. The first method equates horizontal transects of DOC concentration with flow times and uses parallel decay models to uncover components of varying decay rate. The second method uses carbon isotopes to directly infer the age and cycling timescale of DOC. If the deep DOC reservoir is isolated from carbon inputs, the age of deep DOC equals its turnover time, and net fluxes calculated from concentration gradients equal total fluxes. However, it has long been suggested that the deep DOC could instead be in a steady state continuously fed by processes like the dissolution of sinking particles and chemoautotrophy. We use currently available bulk DOC data to suggest that the deep DOC reservoir is in a dynamic steady state and contains a substantial portion of relatively fast cycling material. Three lines of evidence are put forth. First, we suggest that the bulk DOC concentrations in the deep ocean are not monotonically decreasing, suggestive of external inputs. Additionally, using public data from both the Hawaii Ocean Time Series (HOTS) and the Bermuda Ocean Time Series (BATS) we demonstrate that between these two sites the scaled variance increases, also suggestive of external inputs. Second, we apply this knowledge to plots of DOC radiocarbon and concentration, using deviations from linearity in this 'Keeling Space' to suggest penetration of radiocarbon modern material into the deep ocean. Finally, we use the concept of a fast equilibrating reservoir of DOC in the 
deep ocean to explain the anomalous aging of deep water DOC in the Southern Ocean relative to the Sargasso Sea and North Central Pacific deep waters. We use a simplified box model to constrain the real value of deep recalcitrant DOC at less than $-700 \%$ and $27 \mu \mathrm{M}$. Together, these lines of reasoning suggest that deep water DOC may be in a steady state where the lack of large concentration fluctuations is not indicative of small turnover times.

\subsection{Introduction}

Oceanic dissolved organic carbon (DOC) contains as much carbon as Earth's atmosphere yet its cycling timescales and composition remain poorly constrained $[1,18]$. Because a quantitative separation of DOC from the seawater matrix is difficult, the vast majority of information about the cycling of DOC in the ocean comes from measurements of its bulk concentration and isotopic content $[8,12,20]$. Over the last decade, tremendous progress has been made in measuring the bulk DOC concentration across the ocean basins $[13,16,31]$. Unfortunately, the corresponding isotopic information which provides both source and timescale information is three orders of magnitude less densely sampled. Data is especially light for the deep ocean $(\geq 1000$ meters) [10]. Currently, radiocarbon data for DOC in the deep ocean is relegated to seven different regions (Fig. 3-1) which make equating horizontal gradients in deep water DOC with cycling timescales difficult $[9,12,46-48]$.

Despite the lack of good spatial isotopic data for DOC, excellent depth resolution exists at the locations where measurements have been made [12]. These depth profiles have lead credence to the idea that DOC in the ocean consists of two components, distinct in both radiocarbon age and biological lability. Radiocarbon modern, semi-labile DOC, is produced by plankton communities in the surface ocean over a background component of radiocarbon depleted, recalcitrant, carbon which cycles in the ocean over thousands of years and persists in the deep sea $[9,10,12,49]$. This behavior can be understood in terms of a Keeling plot, where a straight line in ra- 

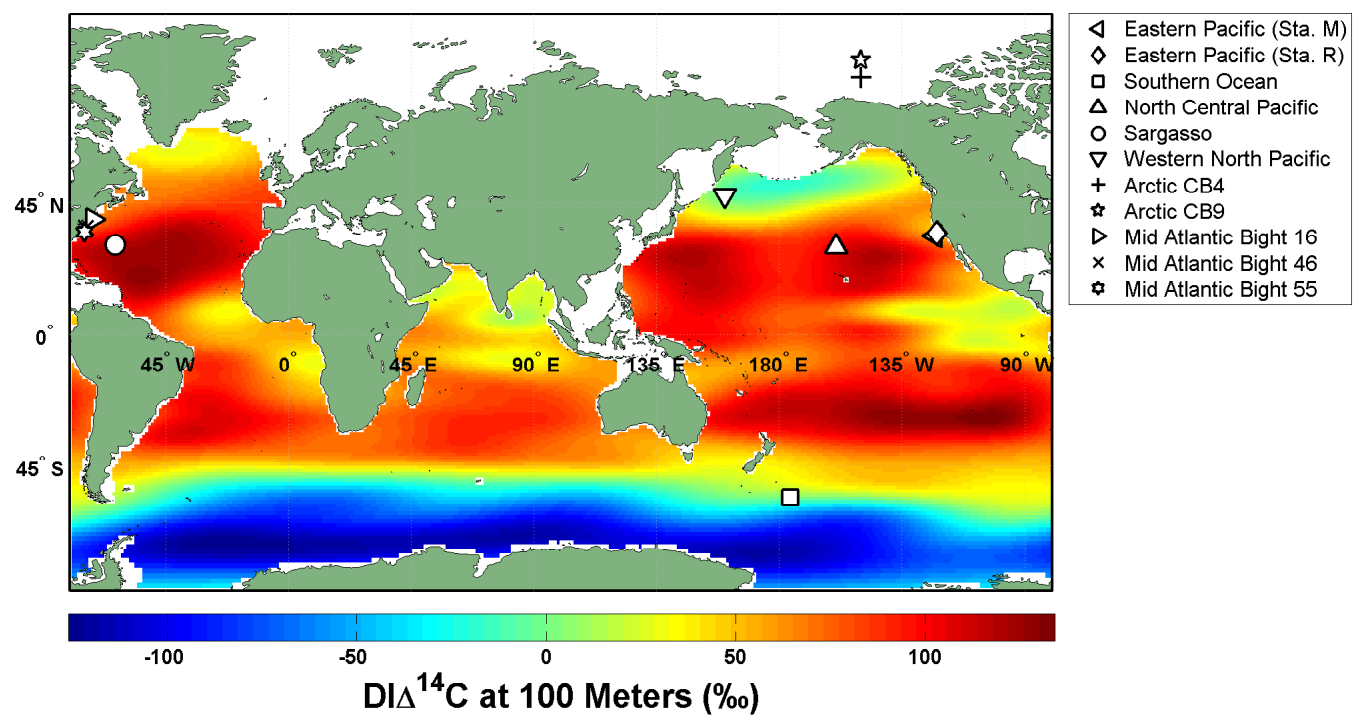

Figure 3-1: The locations of all DOC radiocarbon data with depth greater than or equal to 1000m. This includes the Arctic Ocean [46], Eastern Pacific Ocean [9], Eastern Pacific Slope [9], North Central Pacific Ocean [12], Sargasso Sea [12], Southern Ocean [47] and Western North Pacific Ocean [48]. The background field is an estimate of the $\Delta^{14} \mathrm{C}$ values for dissolved inorganic carbon at 100 meters depth [22] 


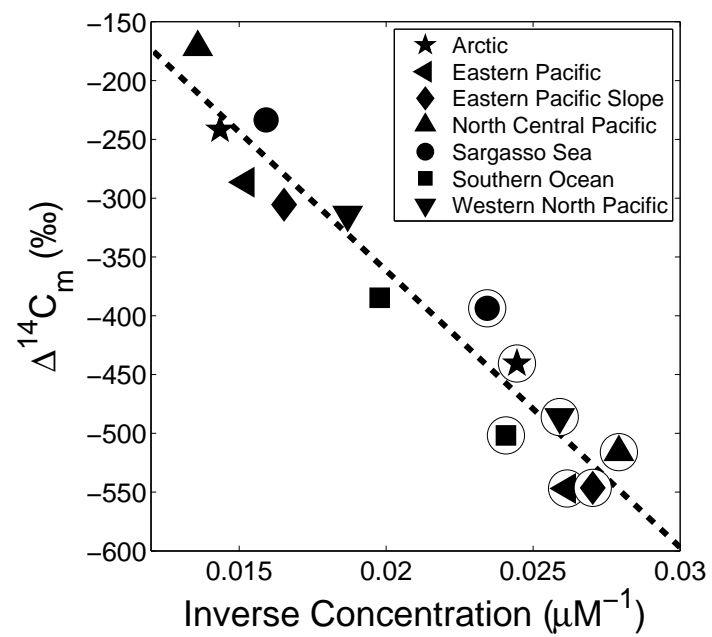

Figure 3-2: Data for depth profiles across multiple oceanic sites is plotted in Keeling Space. The average of all data below 1000 meters is denoted, o, and between 1-100 meters, free symbols, for the Arctic Ocean [46], Eastern Pacific Ocean [9], Eastern Pacific Slope [9], North Central Pacific Ocean [12], Sargasso Sea [12], Southern Ocean [47] and Western North Pacific Ocean [48]. The straight line is a best fit with an intercept commensurate with $\Delta^{14} C_{x s}=70 \%$ and is consistent with the TCM.

diocarbon vs. inverse concentration space yields a straight line for two-component mixing $[10,20]$. The success of the Keeling approach is straightforwardly seen by plotting the average surface $(<100$ meters) and deep $(>1000$ meters $)$ water DOC values in Keeling space for all available open ocean data sets (Fig. 3-2). Despite the extreme variability in conditions between these cites, all data is consistent with a two-component model (TCM) where the measured values, subscript $m$, are combinations of the background recalcitrant fraction, subscript $b k g$, and the more labile excess, subscript $x s$, component (See Section 3.5.1). We find that $\Delta^{14} \mathrm{C}_{x s} \approx 70 \%$, consistent with surface values of $\Delta^{14} \mathrm{C}$ for dissolved inorganic carbon (DIC) [22]. The underlying premiss of the TCM, that depleted carbon from deep waters persists in the surface ocean where enriched DOC is produced, has substantial support from bulk isotope measurements.

Bulk concentration measurements made over the last decade [13, 16,31] are also in agreement with the TCM. These measurements consistently show elevated con- 
centrations of DOC in the surface ocean relative to depth with a gradual decrease of DOC in the deep ocean from about $45 \mu \mathrm{M}$ in the deep North Atlantic to $35 \mu \mathrm{M}$ in the deep North Central Pacific (Fig. 4-4) following the movement of deep water masses. Current attempts to model DOC in the deep ocean consider it to be an isolated reservoir of carbon with different components decaying exponentially at different rates $[13,16,31]$.

The deep ocean background component is estimated to consist of 'Refractory' DOC with a turnover time of $\tau \sim 16000$ years while the surface ocean contains an equal proportion of material with a lifetime of less than 20 years $[13,14,16]$. Estimates for turnover time in these models are found by adjusting the quantity and decay rate until the concentration field is well matched. This process assumes that their are no additional inputs of DOC other than that produced in the surface ocean [16]. However, it does take into consideration the complex flow structure of the ocean. Other turnover time estimates based solely on radiocarbon measurements predict a different turnover time for DOC using the radiocarbon age. Radiocarbon values in the deep North Pacific lead to a radiocarbon age of 6000 years [8], far faster than that predicted from the deep flow field [13]. However, this direct comparison is misleading because even if the reservoir is in a first order steady state, the radiocarbon age will be related to the turnover time through its age distribution [38].

In a well mixed reservoir in first-order steady state, the age distribution, $P(a)$, is related to the turnover time, $\tau$, through the exponential function [38] where

$$
P(a)=\frac{1}{\tau} e^{-a / \tau} .
$$

Dissolved organic carbon in the surface ocean might be a good example of this as inputs of DOC are balanced by export and decay. In the traditional view of the DOC cycle [1], deep water formation isolates the DOC from sources and it undergoes solely 
decay processes. After spending an amount of time, $a_{0}$, in the deep ocean the age distribution will have the same shape, but shift in age so that

$$
P(a)=H\left(a-a_{0}\right) e^{a_{0} / \tau} \frac{1}{\tau} e^{-a / \tau}
$$

where the Heavyside step function $H\left(a-a_{0}\right)$ equals zero before $a_{0}$ and 1 after and including $a_{0}$. It accounts for the minimum age shifting from 0 to $a_{0}$. The age distribution is zero for all time before $a_{0}$ at which point the distribution is exponential. From the age distribution, we can calculate the $\mathrm{C}^{14}$ isotopic ratio

$$
R=\frac{\Delta^{14} \mathrm{C}+1000}{1000}
$$

which depends on age through the radiocarbon decay constant, $\lambda \approx 1 / 8267 \mathrm{yr}^{-1}$, and the radioactive decay equation

$$
R(a)=R(0) e^{-\lambda a}
$$

where $R(0) \approx 1$ (new material with $\Delta^{14} \mathrm{C}=0$ ). The isotopic ratio for the deep Pacific ocean, $R_{p}$, can be calculated by integrating over the weighted age distribution

$$
R_{p}=\int_{0}^{\infty} e^{-\lambda a} H\left(a-a_{0}\right) e^{a_{0} / \tau} \frac{1}{\tau} e^{-a / \tau} d a .
$$

This integral can be solved and reduces to

$$
R_{p}=\frac{1}{\tau \lambda+1} e^{-\lambda a_{0}}
$$

We insert the following values: $\tau=16,000$ years [13], $\lambda \approx 1 / 8267 \mathrm{yr}^{-1}$ [1], and $a_{0}=1000-2000$ years [22]. The value of $a_{0}$ is an estimate for the amount of time deep water DOC in the North Central Pacific [8] has been isolated from surface waters. 
We calculate the deep radiocarbon value of the DOC to be between -700 and $-730 \%$, over 100\% less than that expected for the region [12] or over 2000 years older.

This discrepancy has been found by noting that the radiocarbon age of the deep North Central Pacific Ocean, 6000 years [8], is substantially less than the turnover time predicted by global DOC degradation models [16]. It is assumed that the deviation between the two estimates is solved by the fast removal of old DOC in the surface ocean from ultraviolet radiation $[16,50]$. However, the estimates of this removal suggest a turnover time of $500-2000$ years, which is three times smaller than the radiocarbon age and requires substantial external inputs to the deep ocean [50]. Large external inputs to the deep ocean are incompatible with the ocean mixing models which assume minimal inputs $[13,16]$ making photo-oxidation incapable of rectifying the long turnover times found in models.

An additional method of rectifying the measured values and those predicted from concentration gradients is to pose the existence of a background component of DOC which equilibrates on a fast timescale relative to ocean circulation. Being relatively constant spatially, it would have no measurable impact on the simulations of global DOC concentration. However, if its radiocarbon age is substantially younger, it would explain the discrepancy between the predicted and measured radiocarbon values of bulk DOC. We suggest that this pool consists of material injected to the deep ocean from the dissolution of sinking particles [15]. This mechanism and its potential importance is often discussed $[8,13,16,31]$, but dismissed because there is insufficient evidence that the reservoir is of a meaningful size.

The difficulties in quantifying the importance of such a steady state reservoir come from its small spatial variability. It is straightforward to see the influence of large perturbations like surface production on DOC concentration and radiocarbon values because the influence is both large, and spatially isolated in the surface ocean [51]. However, if portions of the deep ocean DOC reservoir are in steady state, no gradients 
may be observable even if there is tremendous carbon flux through the reservoir. In order to measure the flux through a steady state reservoir, one must either directly measure the flux, measure the age, or have a mechanistic connection between absolute concentration and turnover time.

We provide evidence from global bulk measurements of both DOC concentration and radiocarbon which suggest the presence of a substantial reservoir of deep semilabile organic carbon in the ocean. Because the first order changes in DOC are caused by the large fluctuations in autochthonous surface ocean DOC, we look for evidence in second order effects: small spatial concentration changes, changes in the variability of concentration measurements, deviations from linearity in Keeling space, and spatial correlations in Keeling slope. Together, these observations provide evidence of a semi-labile reservoir of DOC in the deep ocean. The existence of this reservoir can be used to explain the aging of DOC across the three major ocean basins $[12,21]$, and constrain the radiocarbon values of the true background component and deep semi-labile reservoir.

\subsection{Bulk Concentration Data}

If a substantial portion of the deep DOC reservoir is populated by semi-labile material from sinking particles, then we might expect regions of high export production like equatorial regions and the Southern Ocean to see increases in the bulk DOC concentration $[18,52]$. To test this, we re-compile and plot (Fig. 4-4) data following the global circulation of the North Atlantic Deep water $[1,16,53]$. Consistent with the TCM we find a decrease of approximately $10 \mu \mathrm{M}$ between the North Atlantic and North Pacific deep water. However, although the general trend is for decreasing DOC concentrations, there is a clear local increase of $1-2 \mu \mathrm{M}$ in deep DOC concentrations in equatorial regions where there is more export production [54]. Based on other pre- 


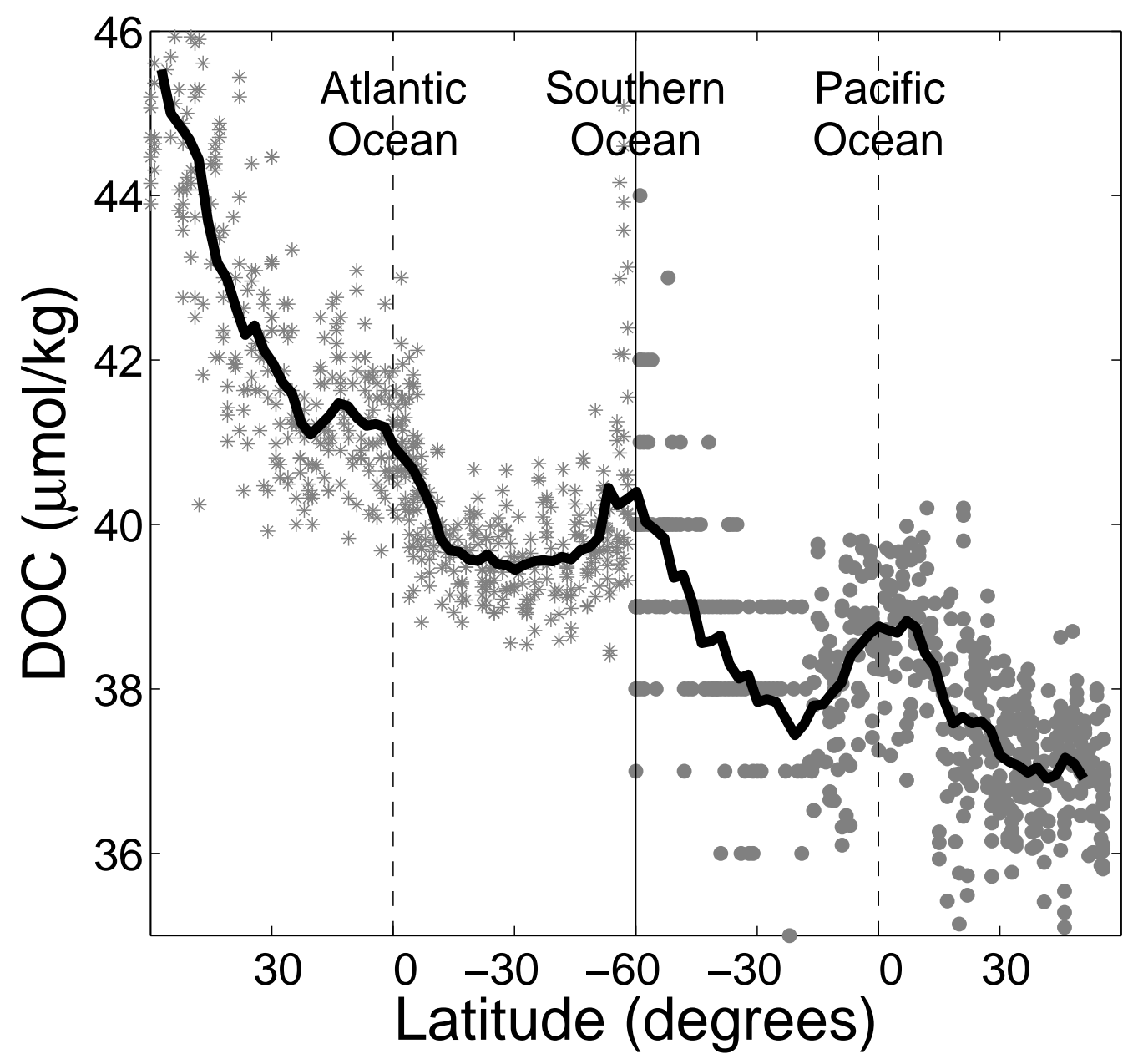

Figure 3-3: DOC concentration $(\mu \mathrm{mol} / \mathrm{kg})[16]$ in the core of the North Atlantic Deep Water along Atlantic transect A16 $(*)$ and in the Circumpolar Deep Water along Pacific transect P16 $(\bullet)$. Water samples were used from the neutral density surfaces consistent with these water masses: $41.25<\sigma_{3000}<41.5$ in the Atlantic and $45.85<\sigma_{4000}$ in the Pacific [16]. The running mean across $15^{\circ} \mathrm{N}-\mathrm{S}$, solid line, is superimposed on the individual DOC measurements, single points. Note the $1-2 \mu \mathrm{M}$ increase in DOC at the equator relative to the background trend in both basins. This is consistent with an increased particle export from enhanced primary production at the equator. 
sented scenarios for a deep semi-labile reservoir of carbon $[8,10]$, this could represent a $10-20 \%$ fluctuation in the semi-labile pool of deep carbon. As deep water DOC slowly moves around the ocean it is exposed to varying levels of particle flux which should add semi-labile carbon to the deep ocean. In this way, the bulk concentration and its derivatives could be a metric of the local activity, rather than a sign of a slow monotonic decay. In fact, if the export production is substantially larger in the North Atlantic relative to the Pacific, even the bulk decrease between the two basins could be representative of changing steady state conditions, rather than slow, monotonic change. The increase of DOC in the Southern Ocean could also be due to particle flux [54], or the deep water mixing at this location physically moving DOC from the surface to deep waters [53].

\subsubsection{Changes in Local Variability}

In addition to changes in the bulk concentration of DOC in the deep ocean, random inputs of carbon from the dissolution of sinking particles should create structure in the local variability of concentration measurements. If recalcitrant DOC is produced in the surface ocean by microbes [49] and is slowly consumed in the deep ocean [55] then the variability of carbon concentration measurements should decrease as a function of the age of a deep water mass. This can be explicitly shown by considering the deep ocean DOC to be a set of exponentially decaying reservoirs of carbon.

Let us define the probability distribution for DOC concentration, $c$, in the deep ocean as a function of the time, $t$, since it was exported from the surface ocean as $P(c, t)$, where the moments of this distribution

$$
\left\langle c^{n}\right\rangle=\int_{0}^{\infty} c^{n} P(c, t) d c .
$$

If DOC decreases exponentially during its ocean transit we can derive a relationship 
between the mean of the distribution, $\langle P(c, t)\rangle$, and its moments. Defining the initial probability distribution as $P_{0}(c)$, we can write the time dependent distribution in terms of $\tilde{c}=c e^{-k t}$

$$
P(c, t)=e^{k t} P_{0}(\tilde{c})
$$

Combining equations (3.7) and (3.8), we find that as DOC decays away

$$
\left\langle\tilde{c}^{n}\right\rangle=\left\langle c^{n}\right\rangle e^{-k t}
$$

so do its moments. Utilizing this formula, we can show that the standard deviation of the distribution, $\sigma=\sqrt{\langle(\tilde{c}-\langle\tilde{c}\rangle)\rangle}$, scaled by its mean value is a constant for all time,

$$
\frac{\sigma}{\langle\tilde{c}\rangle}=\frac{\left(\left\langle c^{2}\right\rangle-\langle c\rangle^{2}\right)^{1 / 2}}{\langle c\rangle} .
$$

This situation represents the current paradigm of slow exponential decay for the deep ocean. In this case we expect the variability in DOC concentration scaled by its mean to be a constant between the deep waters of the Atlantic and Pacific oceans despite the long transit time of deep waters between the two sites. Comparing the distributions of DOC below 2000 meters depth at the Hawaii Ocean Time Series (HOTS) [56] and the Bermuda Atlantic Time Series (BATS) [57] in figure 3-4 allows us to directly test this hypothesis. In the much older deep waters of the Pacific the mean concentration value of DOC is $37.6 \mu \mathrm{M}$, about 5 micromolar less than in the Atlantic Ocean $(43.1 \mu \mathrm{M})$. The variability in the Atlantic is $\sigma=.58 \mu \mathrm{M}$, making the predicted ratio from equation $(3.10) \sigma /\langle\tilde{c}\rangle=.01$. For the Pacific we find this ratio to be five times larger, $\sigma /\langle\tilde{c}\rangle=.05$. We used data from below 2000 meters to avoid issues with surface mixing. Additionally, all data was taken from after 2003, to guarantee the use of intercalibration standards [58].

If the deep ocean monotonically ages, the increasing variance between the Atlantic and Pacific locations requires that important additional sources of DOC feed the deep 


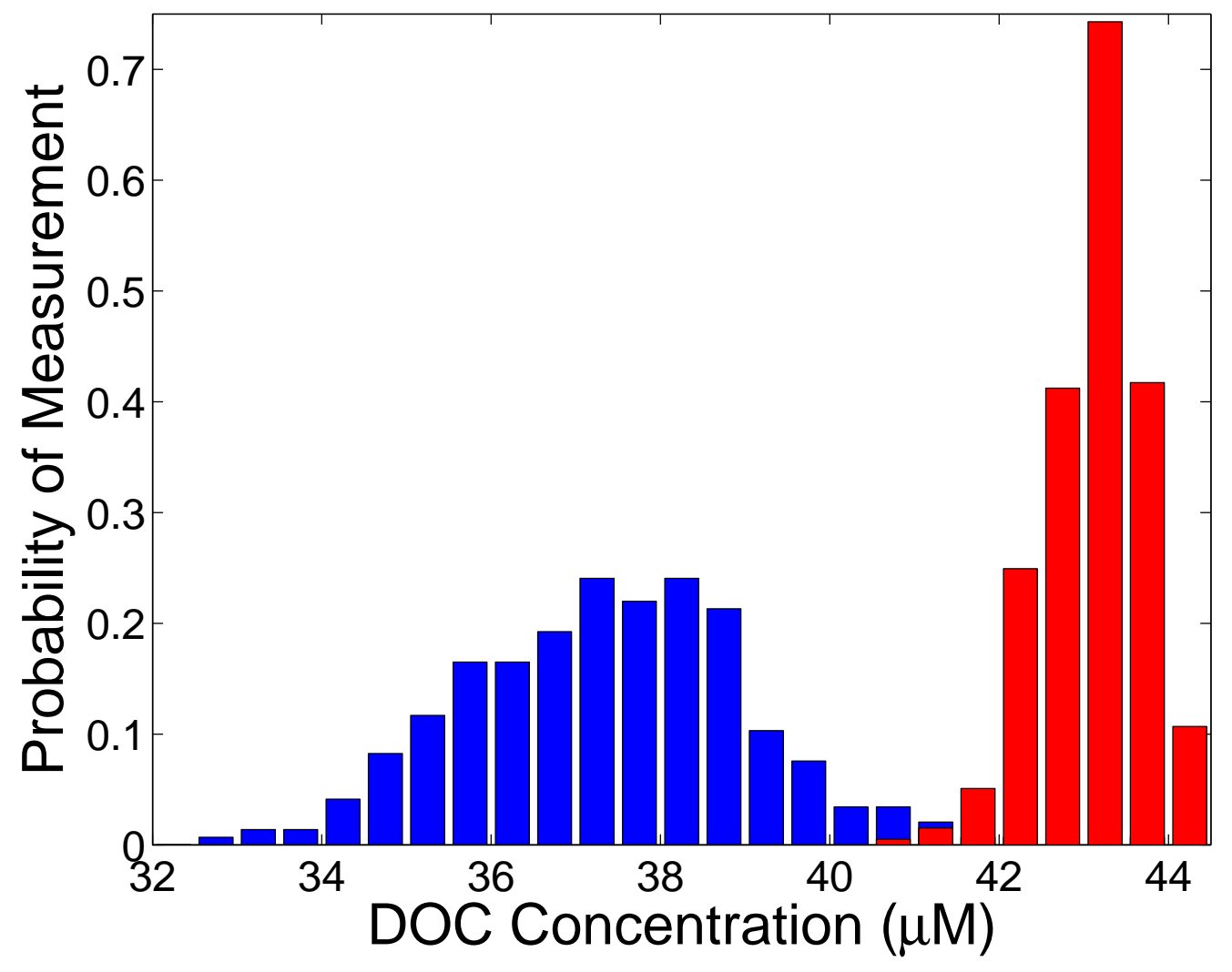

Figure 3-4: DOC variability: Histograms of measurements taken on DOC below 2000 meters at the HOTS [56] (blue) and BATS [57] (red) long term ocean monitoring sites in the Pacific and Atlantic oceans respectively since 2003. Although the mean concentration value in the Pacific Ocean $(37.6 \mu \mathrm{M})$ is more than five micromolar less than in the Atlantic the standard deviation in the Pacific $(\sigma=1.7 \mu \mathrm{M})$ is more than three times as large as the deep Atlantic $(\sigma=.58 \mu \mathrm{M})$. 
ocean. We suggest that particle export and dissolution may play a role [15].

This increase in variance is consistent with the patterns of surface primary production combined with the flow of deep water throughout the deep ocean $[54,59,60]$. As deep water moves south from the North Atlantic it moves underneath a fairly constant surface production leading to the high mean value we see and low variance. At the Pacific site, deep water must flow under a narrow band of high primary production and particle flux at the equator which could be responsible for the additional variability seen at the Pacific site [54].

These ideas are corroborated when we plot an estimate for the local variance as a function of the spatial location using the data in figure 4-4 [16]. The variance decreases moving south in the deep Atlantic and plateaus at the equator south of the primary production maxima. Mixing reintroduces variance in the Southern Ocean [53] which decays moving north before encountering another factor of two shift right after the concentration maxima (between 0 and $30^{\circ} \mathrm{N}$ ) coincident with the high variance seen at HOTS. Comparing the absolute values between the transect and timeseries data is difficult as the potential sources of the variability is different. Additionally, the location of the Atlantic Ocean Data [16] is in the Eastern Atlantic whereas BATS is in the Sargasso Sea making a direct comparison especially problematic. However, the general fluctuations in the variance in figure 3-5 are consistent with the time-series data $[56,57]$, showing that the increased variance moving northward from the equator in the Pacific is unlikely to be an artifact.

One important thing to note is that changes in the average concentration and variance appear correlated, yet not in phase. For example, the maxima in the variance occurs north of the equator while the concentration maxima (see Fig. 4-4) occurs at the equator. We demonstrate in the following section that while the integrated inputs are responsible for setting the steady state average concentration, the variability in the size of the inputs sets the variance, consistent with the idea that changing variance 


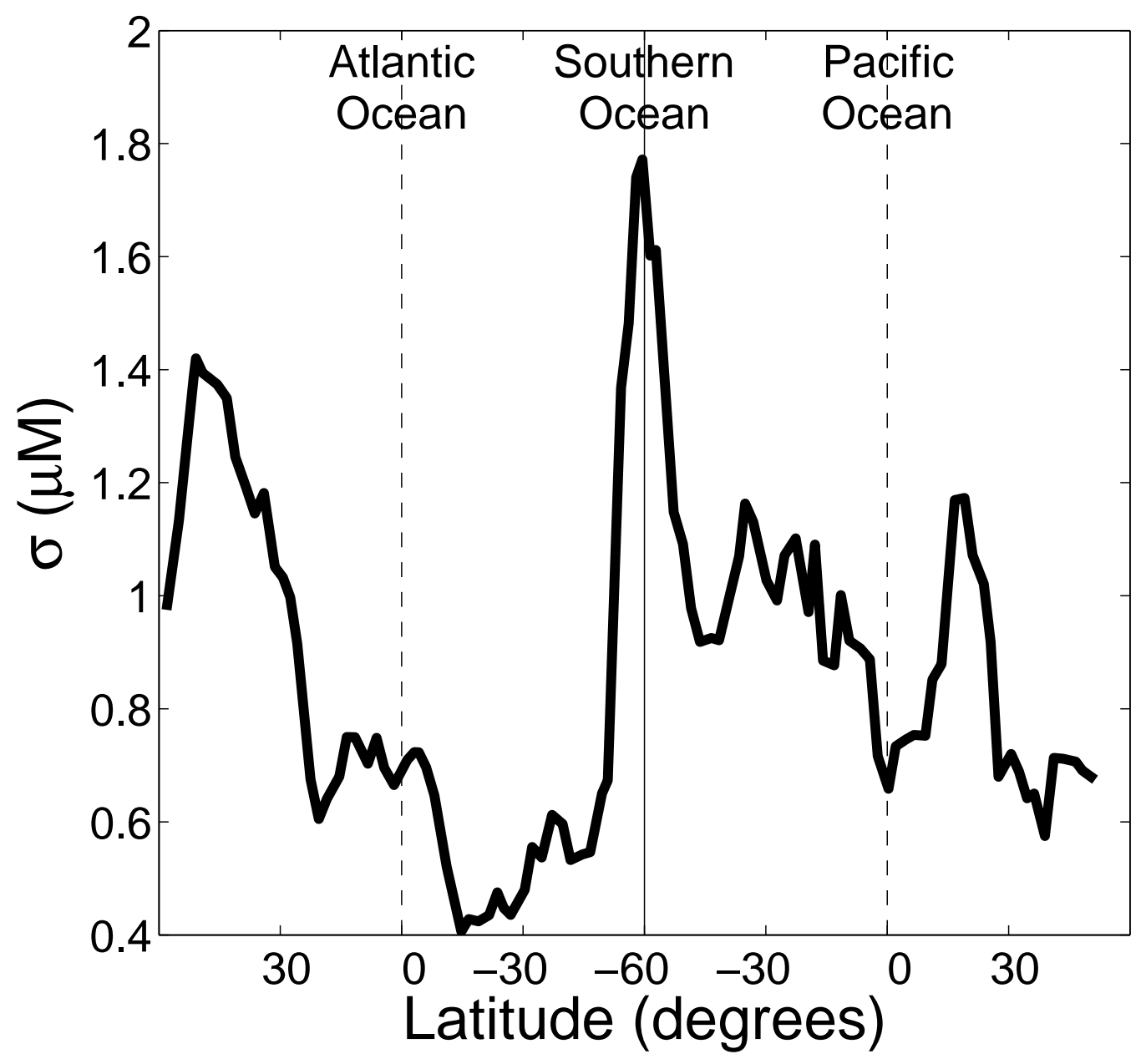

Figure 3-5: The standard deviation $\sigma$ of DOC concentration $(\mu \mathrm{mol} / \mathrm{kg})[16]$ in the core of the North Atlantic Deep Water along Atlantic transect A16 $\left(^{*}\right)$ and in the Circumpolar Deep Water along Pacific transect P16 $(\bullet)$. Water samples were used from the neutral density surfaces consistent with these water masses: $41.25<\sigma_{3000}<$ 41.5 in the Atlantic and $45.85<\sigma_{4000}$ in the Pacific [16]. Variance is calculated from a moving window across $10^{\circ} \mathrm{N}-\mathrm{S}$. 
is associated with changing flux conditions rather than their absolute value.

\subsubsection{Application of Random Inputs}

The assumption of steady state conditions for the deep ocean DOC reservoir presumes that the deep reservoir is continually fed on the timescale of decay. This can be accomplished by replenishment in the surface ocean if the equilibration times are thousands of years, or by continual replenishment. If random events like particle export feed the deep ocean DOC, then we can model the fluctuating portion of DOC in a water parcel, $C(t)$, as the summation of random exponentially decaying pulses,

$$
C(t)=\sum_{i=1}^{\infty} F\left(t-t_{i}\right)
$$

Here,

$$
F(t-t i)=\eta e^{-k\left(t-t_{i}\right)} H\left(t-t_{i}\right),
$$

where $\eta$ is the magnitude of the pulse, $H\left(t-t_{i}\right)$ is the Heaviside step function, and $k$ is the decay constant. Setting $\nu$ equal to the number of pulses per unit time, we invoke Campbell's theorem [17]

$$
\langle C(t)\rangle=\nu \int_{-\infty}^{\infty} F(t) d t
$$

and

$$
\left\langle(C(t)-\langle C(t)\rangle)^{2}\right\rangle=\nu \int_{-\infty}^{\infty} F^{2}(t) d t .
$$

For random exponential pulses, the ratio of the standard deviation to the mean depends solely on the pulse magnitude, and we find that

$$
\frac{\sigma}{\langle C(t)\rangle}=\left(\frac{\eta}{4}\right)^{1 / 2} .
$$


If the deep DOC is fed by random decaying inputs, the normalized standard deviation depends entirely on the magnitude of the pulses, not their integrated flux.

Although it is tempting to suggest that the increased variance is due to a decreased particle flux, this result suggests instead that the variability is determined by the absolute magnitude of the input pulses. Thus, the change in variance between the two sites suggests a decrease in total inputs, yet an increase in their magnitude. This is consistent with the high steady flux in the North Atlantic and the large variability in flux in the Pacific ocean moving northward across the equator $[54,59,60]$.

\subsection{Radiocarbon Data}

Combining concentration measurements with the much sparser and more difficult radiocarbon measurements allows the possibility of connecting a concentration measurement with a turnover time $[8,10,12]$. In the beginning of this manuscript we discussed how depth profiles plotted in 'Keeling Space' yield straight lines suggesting the combination of a surface excess component with a recalcitrant background component [20]. However, because there is a time gradient between the surface and deep waters, it would be expected that fluctuations in the surface radiocarbon values of DIC could create changes in both the slope and curvature of depth profile data in Keeling space [9].

Spatially, the $\Delta^{14} \mathrm{C}$ value of surface DIC covers a large range from less than -100 to greater than $150 \%$ [22], due to a balance between the flux of radiocarbon enriched carbon dioxide from the atmosphere and the upwelling of radiocarbon depleted carbon from deeper waters. Semi-labile material from the surface ocean also has a large range of $\Delta^{14} \mathrm{C}$ values consistent with this variability. If a large semi-labile reservoir of material exists in the deep ocean, its value should track that of the surface $\mathrm{DI} \Delta^{14} \mathrm{C}$ as DOC in the ocean is believed to originate in the surface ocean $[12,49]$. Temporally, 
there has also been a large shift in the surface values of $\mathrm{DI} \Delta^{14} \mathrm{C}$ due to nuclear bomb testing (Fig. 3-12). In the absence of radiocarbon additions to the system, new material has a $\Delta^{14} \mathrm{C} \approx 0 \%$. Nuclear bomb testing created a tremendous amount of radioactive carbon and drove this value up to over $150 \%$ [19,61]. These fluctuations must propagate through the water column. This propagation should be observable, and usable as a probe to test the age structure of deep ocean DOC.

\subsubsection{Curvature in Keeling Space}

Keeling curves are linear because all concentrations can be written as a summation of a fluctuating excess component with a constant radiocarbon value, over a background component of constant radiocarbon value and concentration $[10,20]$. In practice, these varying concentrations are generated from depth profiles where concentration decreases with depth (away from surface production) and bulk age increases [10]. Injections of material which are uncorrelated with concentration, and depth, will shift the slope of a Keeling line (Fig. 3-6) but neither change its intercept or add curvature. These uncorrelated processes act the same as changing the values of the uniform background component.

Generating curvature under the auspices of the TCM requires that the radiocarbon value of the excess component change systematically with depth or bulk concentration. In practice, this could be caused by the addition of a third, non-uniformly distributed DOC component, or by time dependence in the radiocarbon values of injected excess DOC. This can be seen mathematically through the definition of the Keeling Slope

$$
\Psi=C_{b k g}\left(\Delta^{14} C_{b k g}-\Delta^{14} C_{x s}\right)
$$

in the TCM (See section 3.5.1). Independent fluctuations in either $C_{b k g}, \Delta^{14} C_{b k g}$, or $\Delta^{14} C_{x s}$ will change $\Psi$ independently of the concentration $C_{m}$. In order for curvature 


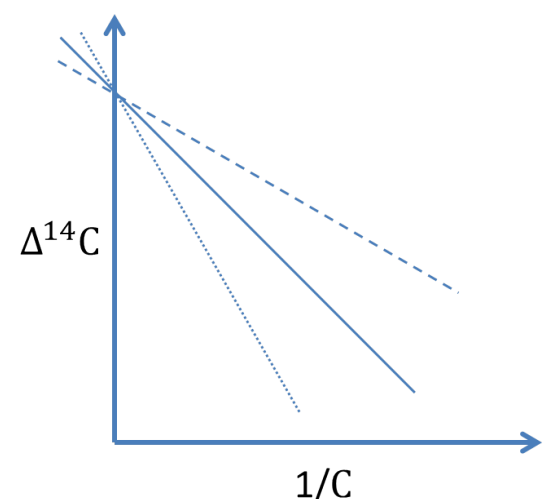

$1 / \mathrm{C}$

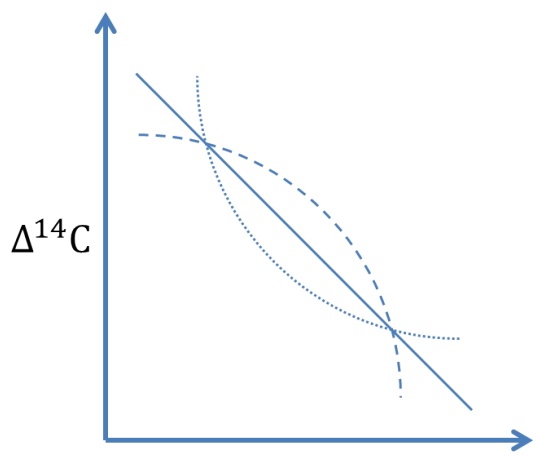

$1 / \mathrm{C}$

Figure 3-6: Uniform vs. Nonuniform Change: A schematic of the effects of uniform changes (left) and monotonic changes (right) to DOC depth profiles (solid line) in Keeling space. (left) Uniform changes shift the slope, but not the intercept in Keeling space. (right) Monotonic changes will yield either positive $(--)$ or negative $(\cdots)$ curvature.

to exist, the Keeling slope must be a function of the bulk DOC concentration, $\Psi\left(C_{m}\right)$. Since bulk concentration tends to decrease monotonically with depth [12], curvature is created by processes which also have depth dependence. We define positive curvature as the Keeling slope increasing with increasing concentration (right to left on a Keeling plot). Negative curvature is defined as having an increasing slope with decreasing concentration (left to right on a Keeling plot).

One likely mechanism for generating curvature is the $\sim 200 \%$ perturbation in surface ocean radiocarbon values due to nuclear bomb testing [19]. As surface ocean $\mathrm{DI} \Delta^{14} \mathrm{C}$ values increase they should induce a negative curvature in Keeling space as radiocarbon values for the high concentration values will increase independently of the rest of the Keeling data. A decrease should cause a positive curvature (Fig. 3-7). Surface ocean radiocarbon DIC values experienced a sharp increase between 19601970 and has bean decreasing approximately linearly ever since. Depending on the equilibrium time $\tau$ of the DOC with the bomb carbon, depth profiles should show curvature due to the rise, fall, or both the rise and continuing fall in surface ocean $\mathrm{DI} \Delta^{14} \mathrm{C}$ measurements. 

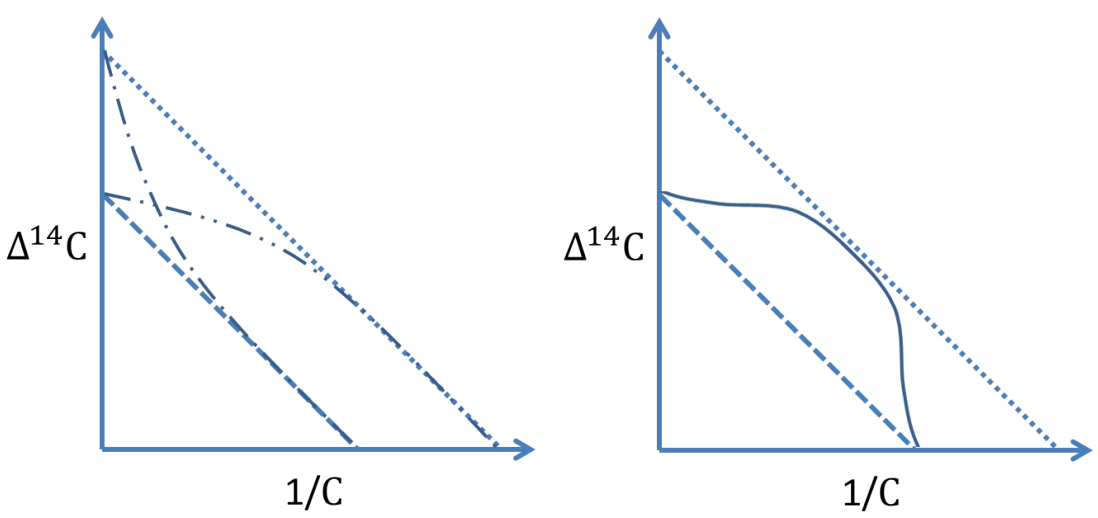

Figure 3-7: Two schematics of fully equilibrated Keeling curves for low (--) and high $(\cdots)$ surface DI $\Delta^{14}$ Cas bounds for additional processes. (Left) A sudden increase $(-\cdot)$ or decrease $(-\cdot \cdot)$ in surface DI $\Delta{ }^{14}$ Ccauses either negative or positive curvature respectively. (right) The increase and decrease of the bomb spike may appear in Keeling space (solid line) if not fully equilibrated.

Other processes may play an important role in generating Keeling space curvature. Mesopelagic chemoautotrophy, for example [62], should cause negative curvature in Keeling space as local DI ${ }^{14} \mathrm{C}$ values are substantially higher than deep water DO $\Delta^{14} \mathrm{C}$ yet lower than surface water DI $\Delta^{14} \mathrm{C}$ values [12]. It has recently been shown that mixing has an important role in the linearization of Keeling curves [10], as the mixing of water-masses with differing $\mathrm{DO} \Delta^{14} \mathrm{C}$ values would produce a depth profile which plotted as a line in Keeling space. With this mechanism in mind, it is important to note that the movement of DOC from regions underlying high DI $\Delta{ }^{14} \mathrm{C}$ surface water to regions of low DI $\Delta^{14} \mathrm{C}$ surface water should create positive curvature, and the inverse process negative curvature. This process could be important in areas like the Southern Ocean where surface $\mathrm{DI} \Delta^{14} \mathrm{C}$ values change by as much as $10 \%$ per degree of north-south movement and deep currents migrate by as much as 1 degree per year $[22,63]$.

The simplest way to test curvature in Keeling space is to look at the difference between the estimate for Keeling intercept, the estimate of the excess component, for the surface ocean section of the Keeling plot when compared with the estimate based 
on the entire depth profile. Starting at the surface we plot the initial portions of Keeling space for the five open ocean sites; Sargasso Sea [12], North Central Pacific [12], Eastern Pacific [9,64,65], Southern Ocean [12], and the Western Pacific Ocean [48](see Figs. 3-8 and 3-9). These sites are the most isolated from external inputs of DOC although external inputs are invoked in both the Eastern Pacific and Western Pacific $[48,65]$. Depth cutoffs were chosen to provide enough of the Keeling plot to generate linearity.

Data was fit using a reduced major axis (RMA) fitting method to account for errors in both axes while RMA errors were taken taken as the errors from using ordinary least squares [20]. All the sites have a statistically different estimate for the $\Delta^{14} \mathrm{C}_{x s}$ value when we consider only surface data as opposed to all of the data at a location (see figs. 3-8 and 3-9 and table 3.1). The Sargasso Sea, North Central Pacific, and Eastern Pacific all exhibit positive curvature, while the Southern Ocean and Western Pacific exhibit negative curvature.

For all locations the estimate for $\Delta^{14} \mathrm{C}_{x s}$ from the surface data is significantly different than the radiocarbon from surface inorganic carbon. Locations with positive curvature predict $\Delta^{14} \mathrm{C}_{x s}$ values which are too low compared with surface DIC, while the values predicted for the negative curvature sites are larger than those ever found in the ocean [19]. These results are consistent with a $\Delta^{14} \mathrm{C}_{x s}$ signal which changes in depth and time. The positive curvature could be caused by the slow decrease in surface DIC radiocarbon [19], while the negative curvature in at least the Southern Ocean is predicted by the northward flow of deep water beneath large gradients in surface radiocarbon $[22,63]$. We can approach the curvature question more objectively by looking for the best smooth curve through the DOC data.

Quantifying the curvature in Keeling space requires a method of estimating the best smooth curve though the data cloud. This poses a few challenges. Firstly, the data contains errors in both the horizontal and vertical axes. For a line, this problem 

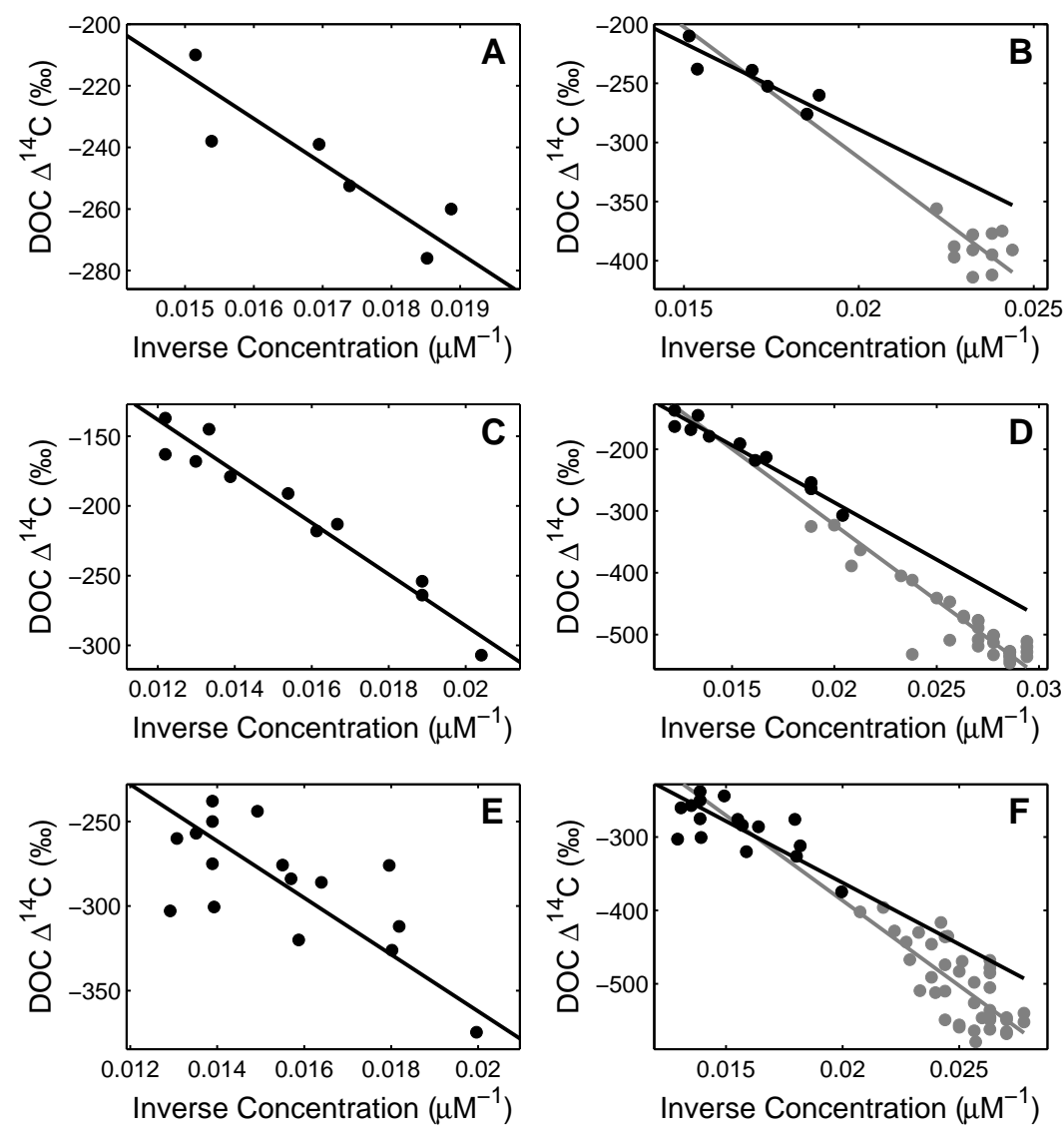

Figure 3-8: Keeling plots for sites where the estimate of $\Delta^{14} \mathrm{C}_{x s}$ increases with depth (positive curvature). Plots A, C, and E are Keeling plots from surface waters whereas plots B, D, and E are from all data. Data is from the Sargasso Sea (A and B) [12], the North Central Pacific (E and F) [12], and the Eastern Pacific [9,64,65]. The depth cutoff for the plots at left is 500 meters (A), 200 meters (C), and 200 meters (E). The filled points are the surface data, and grey the deeper waters. $\Delta^{14} \mathrm{C}_{x s}$ values are estimated as the intercept for the surface (black line) and total (grey line) data. $\Delta^{14} \mathrm{C}_{x s}$ values calculated from the surface data are all significantly different than those calculated from the entire data set $(\mathrm{P}<.05)[20]$. 

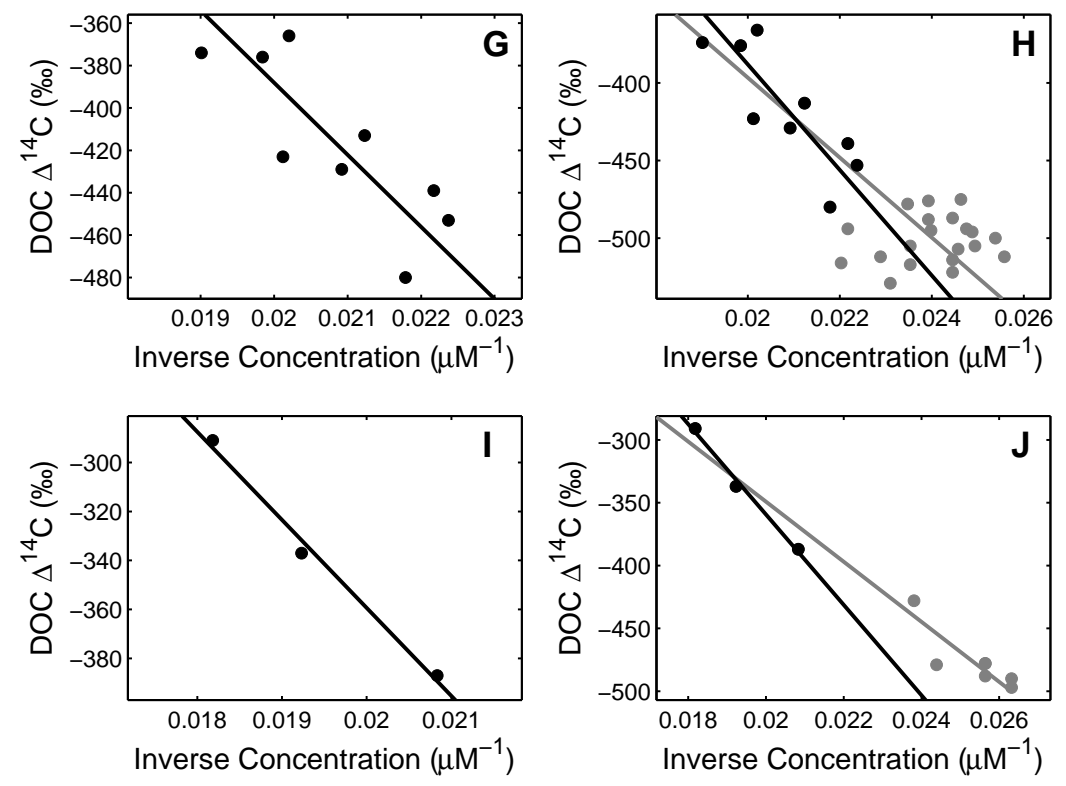

Figure 3-9: Keeling plots for sites where the estimate of $\Delta^{14} \mathrm{C}_{x s}$ decreases with depth (negative curvature). Plots G, and I are Keeling plots from surface waters whereas plots $\mathrm{H}$ and $\mathrm{J}$ are from all data. Data is from the Southern Ocean ( $\mathrm{G}$ and $\mathrm{H}$ ) [12] and the Western Pacific Ocean [48]. The depth cutoff for the plots at left is 750 meters $(\mathrm{G})$ and 200 meters $(\mathrm{I})$. The filled points are the surface data, and grey the deeper waters. $\Delta^{14} \mathrm{C}_{x s}$ values are estimated as the intercept for the surface (black line) and total (grey line) data. $\Delta^{14} \mathrm{C}_{x s}$ values calculated from the surface data are all significantly different than those calculated from the entire data set $(\mathrm{P}<.05)[20]$.

Table 3.1: Estimates of the $\Delta^{14} \mathrm{C}_{x s}$ based on Keeling plots from either the surface ocean or entire depth profile (see Figs. 3-8, 3-9 and table 3.1. The fitting is done with a reduced major axis approach to account for errors in both axes with the standard error on the intercept taken to be the same as for ordinary least squares [20]. At all five of these locations the two estimates are statistically different from each other $(\mathrm{P}<.05[20])$ suggesting that there is significant curvature at these sites.

\begin{tabular}{|r|r|r|r|r|r|r|r|}
\hline Location & Figs. 3-8, 3-9 & Surface Depth & $\Delta^{14} \mathrm{C}_{x s}$ (Surface) & Error & $\Delta^{14} \mathrm{C}_{x s}$ (Total) & Error & Ref. \\
\hline Sargasso Sea & A,B & 500 meters & $3 \%$ & 60 & $129 \%$ & 35 & {$[12]$} \\
\hline North Central Pacific & C,D & 200 meters & $84 \%$ & 26 & $168 \%$ & 32 & {$[12]$} \\
\hline Eastern Pacific & E,F & 200 meters & $-27 \%$ & 57 & $77 \% 0$ & 42 & {$[9]$} \\
\hline Southern Ocean & G,H & 750 meters & $292 \%$ & 155 & $119 \%$ & 72 & {$[47]$} \\
\hline Western Pacific & I,J & 200 meters & $360 \%$ & 67 & $129 \%$ & 38 & {$[48]$} \\
\hline
\end{tabular}


can be dealt with using reduced major axis approaches [20], but this method is not appropriate for higher order curves. The second problem is uneven data spacing. Since the data is collected roughly linearly in depth, the data density across Keeling space is not even, providing more weight to some regions over others. This nonlinearity poses an additional problem for model fitting. If the errors in concentration and radiocarbon are roughly constant $( \pm 1 \mu \mathrm{M}$ and $\pm 10 \%$ respectively [12]), then, when projected into Keeling space, the horizontal errors are both asymmetric and a function of concentration.

In an attempt to mitigate these issues we turned to a clustering algorithm to reduce the degrees of freedom in the data. This was accomplished in two steps. First, the data was rescaled by an estimate of both the horizontal and vertical error range $(2 \mu \mathrm{M}$ and $20 \%$ respectively [12]) to weight each axis equally. Then, a clustering algorithm was applied to split the data into a set number of groups which minimize the distance between data points in each group (K-means method [66]). We tested this method by comparing the estimates of the Keeling intercept for all open ocean sites (excluding the Arctic [46] and Mid-Atlantic Bight [64]) using a best fit line (reduced major axis method [20]) to the intercepts found by drawing a line between the two clusters which best fit the linear data projected in Keeling space. Errors were determined using bootstrapping [67]. Both methods provide the same intercepts within the error bounds, but the clustering methodology allows us to estimate the direction of the curvature by including a third cluster. Additionally, the clustering method avoids the propagation of nonlinear errors into estimation of the Keeling intercept (see section 3.5.2).

In order to quantify the curvature in Keeling space we used the K-means clustering algorithm discussed above to split DOC data from each of the sites into three groups. Each group is defined by a single point which is the center of the cluster. These centers are then connected with a smooth interpolating spline to estimate the best 


\begin{tabular}{|l|l|l|l|l|l|l|}
\hline & $\begin{array}{l}\text { RMA } \\
(\%)\end{array}$ & $\begin{array}{l}\text { Error } \\
(\%)\end{array}$ & $\begin{array}{l}\text { Cluster } \\
(\%)\end{array}$ & $\begin{array}{l}\text { Error } \\
(\%)\end{array}$ & $\begin{array}{l}\text { DI } \Delta^{14} \mathbf{C} \\
(\%)\end{array}$ & $\begin{array}{l}\text { Error } \\
(\%)\end{array}$ \\
\hline Sargasso Sea & 128 & 31 & 129 & 32 & 120 & 3 \\
\hline North Central Pacific & 160 & 14 & 168 & 19 & 136 & 5 \\
\hline Eastern Pacific & 53 & 23 & 77 & 23 & 52 & 5 \\
\hline Southern Ocean & 178 & 57 & 119 & 79 & 14 & 5 \\
\hline Western North Pacific & 108 & 51 & 129 & 64 & -53 & 5 \\
\hline
\end{tabular}

Table 1: Estimates for the radiocarbon value of surface DOC based on RMA fitting and two cluster fitting using bootstrapping for statistics [67]. Data are taken from the published literature $[9,12,47,48]$. An estimate of the mean radiocarbon value of surface ocean DIC is included for comparison. Note that both estimation procedures are within errors of each other, lending credence to the clustering approach.

curve as a function of depth. Three clusters were chosen as the smallest number of points on a plane through which a curve would have curvature. Depth profiles in Keeling space exhibited either negative curvature (Southern Ocean, Western North Pacific), or positive curvature (North Central Pacific, Sargasso Sea, Eastern Pacific) (see Fig. 3-11).

We test the significance of these curvature estimates using a null hypothesis. Data was assumed to fit a two component model with no curvature. We collapsed the data for each site onto the best fit line and then perturbed it by adding random measurement errors in both concentration and radiocarbon. For each instance of the data, we tested whether the magnitude of the measured curvature on the fake data was greater than our measurement. This magnitude was measured by finding the area contained in the triangle defined by the three cluster centers (cross product). These are the $\mathrm{p}$ values found in table 2 .

For profiles with a significant curvature, we can use the assumptions of the TCM to estimate a profile of the excess component. According to the TCM, all samples in a depth profile should contain the same quantity of background carbon. If we assume that the deep ocean represents the value of the background component, then this component can be subtracted to find the concentration and radiocarbon values of the excess component. We estimate the background component as the centroid 


\begin{tabular}{|l|l|}
\hline & P-value \\
\hline Sargasso Sea & .02 \\
\hline North Central Pacific & .91 \\
\hline Eastern Pacific & 0 \\
\hline Southern Ocean & .1 \\
\hline Western North Pacific & .61 \\
\hline
\end{tabular}

Table 2: Estimates of the significance of our curvature prediction. $\mathrm{P}$ values are the fraction of times one is expected to get a curvature of the one measured based on random chance. Values were calculated by adding random errors to data which exactly follows the two component model. We have greater than $90 \%$ confidence that the Eastern Pacific, Sargasso Sea, and Southern Ocean have curvature based on the cluster method used here.

of the deep water cluster and subtract it from the best fit spline. These estimates are plotted in the right side of figure 3-11. What we see from this analysis is that there are significant changes in the radiocarbon constituents of DOC as a function of depth. For all three cases with significant curvature we find that the estimates for the excess component are greater than 300\%, with some estimates along the curve being well higher, as high as 600\%, than anything found in nature. Staying within the confines of the TCM, this suggests either that there is a depth-time dependence on the excess component of DOC across these profiles or that the depth profile is externally influenced. While it is straightforward to use time-dependance and nuclear bomb testing to explain the positive curvature of the Eastern Pacific and Sargasso Sea data, it seems most likely that the negative curvature of the Southern Ocean is due to external influences.

The rise and continual fall of DI $\Delta^{14} \mathrm{C}$ in the surface ocean (Fig. 3-12) is consistent with with the positive curvature seen in the Keeling curves for the Sargasso Sea and Eastern Pacific. Returning to figure 3-6, the keeling curves at both of these sites are consistent with the schematic at the right. The estimate of the background component starts below the best estimate for surface ocean DI $\Delta^{14} \mathrm{C}$ at the site (see table 1 ) and ends higher than the estimate for the highest surface DI ${ }^{14} \mathrm{C}$ values observed over the last hundred years [19]. If the background component of DOC is equal to the 

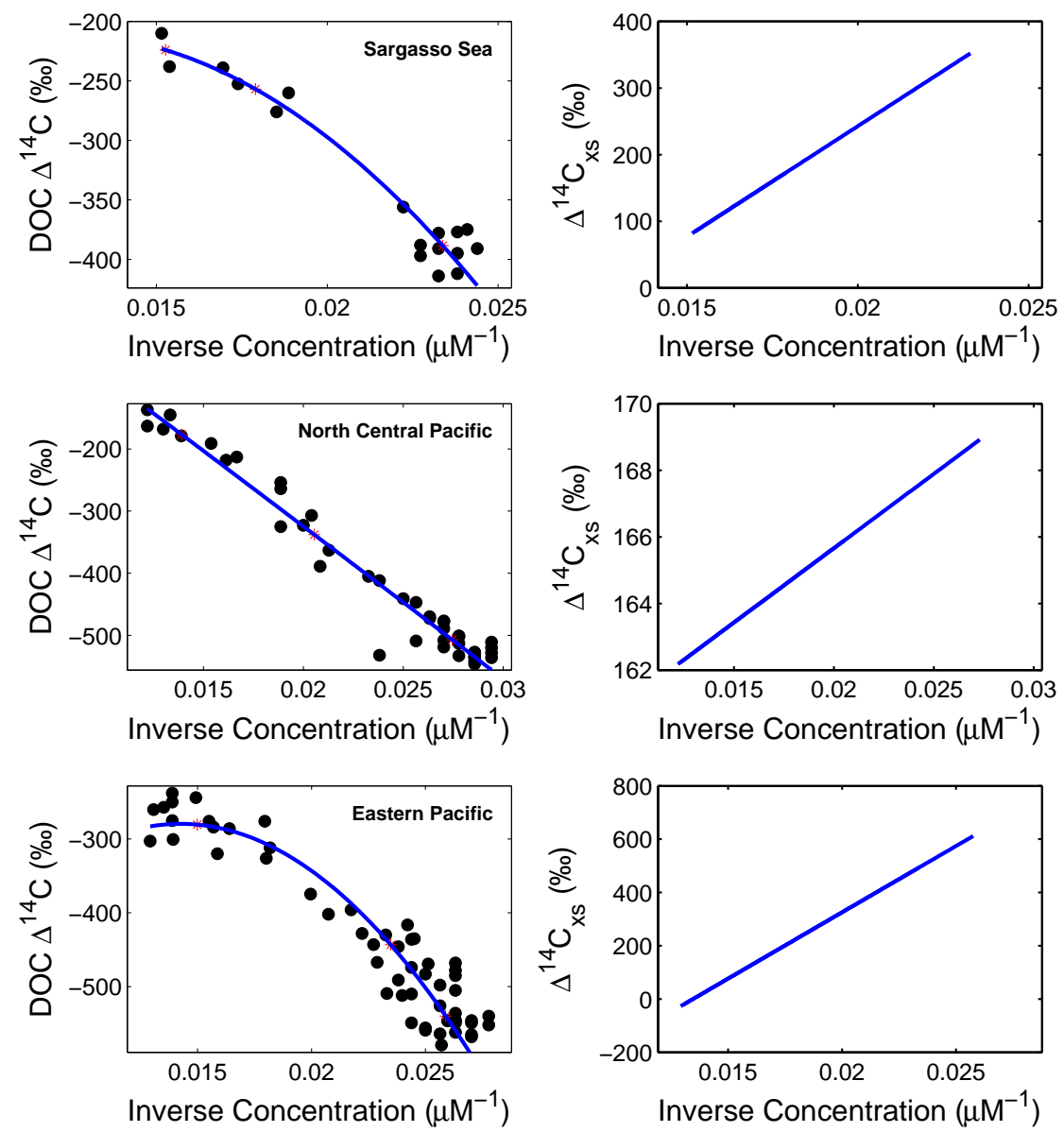

Figure 3-10: Sites with positive curvature. The best fit curves in Keeling space (left) are plotted by fitting a cubic spline through the three best fit clusters in the data. The best estimate of the surface component (right) is taken as the intercept between the cluster with the lowest concentration and each location on the smooth curve. Data is from the Sargasso Sea [12], the North Central Pacific [12], and the Eastern Pacific $[9,64,65]$. 

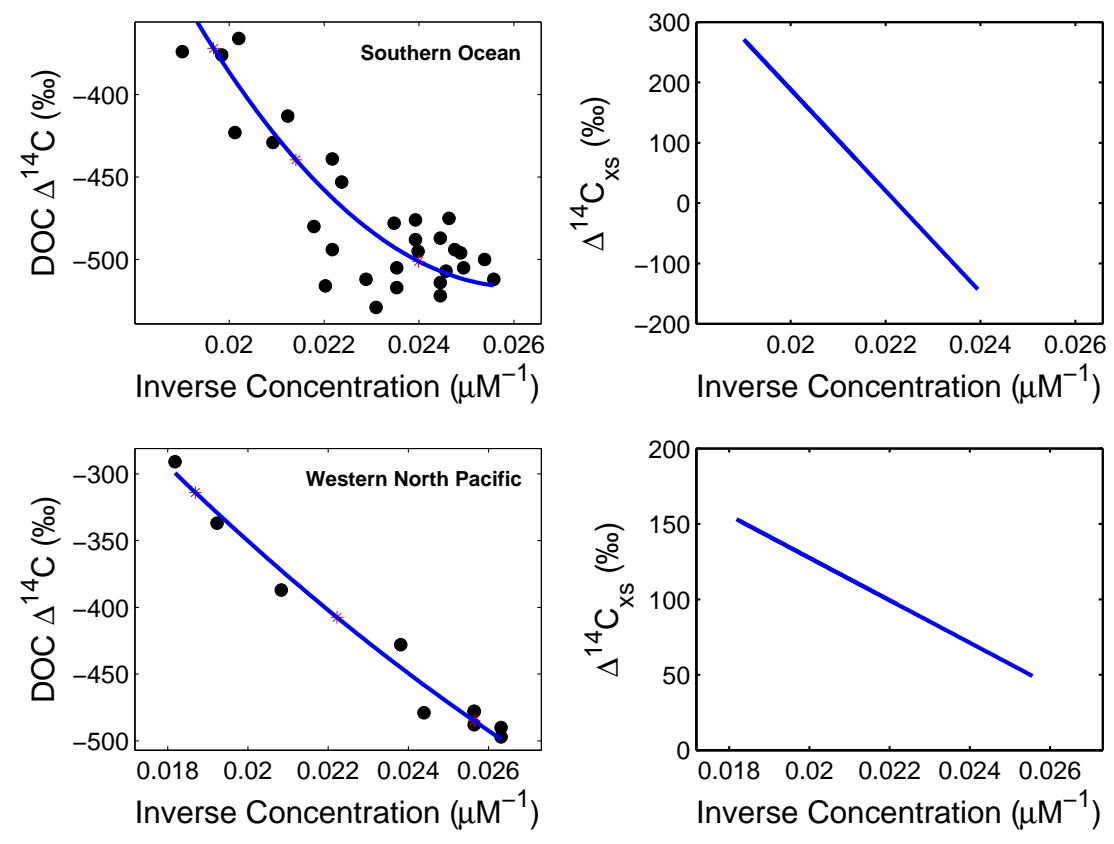

Figure 3-11: Sites with negative curvature. The best fit curves in Keeling space (left) are plotted by fitting a cubic spline through the three best fit clusters in the data. The best estimate of the surface component (right) is taken as the intercept between the cluster with the lowest concentration and each location on the smooth curve. Data is from the Southern Ocean [12] and the Western Pacific Ocean [48]. 


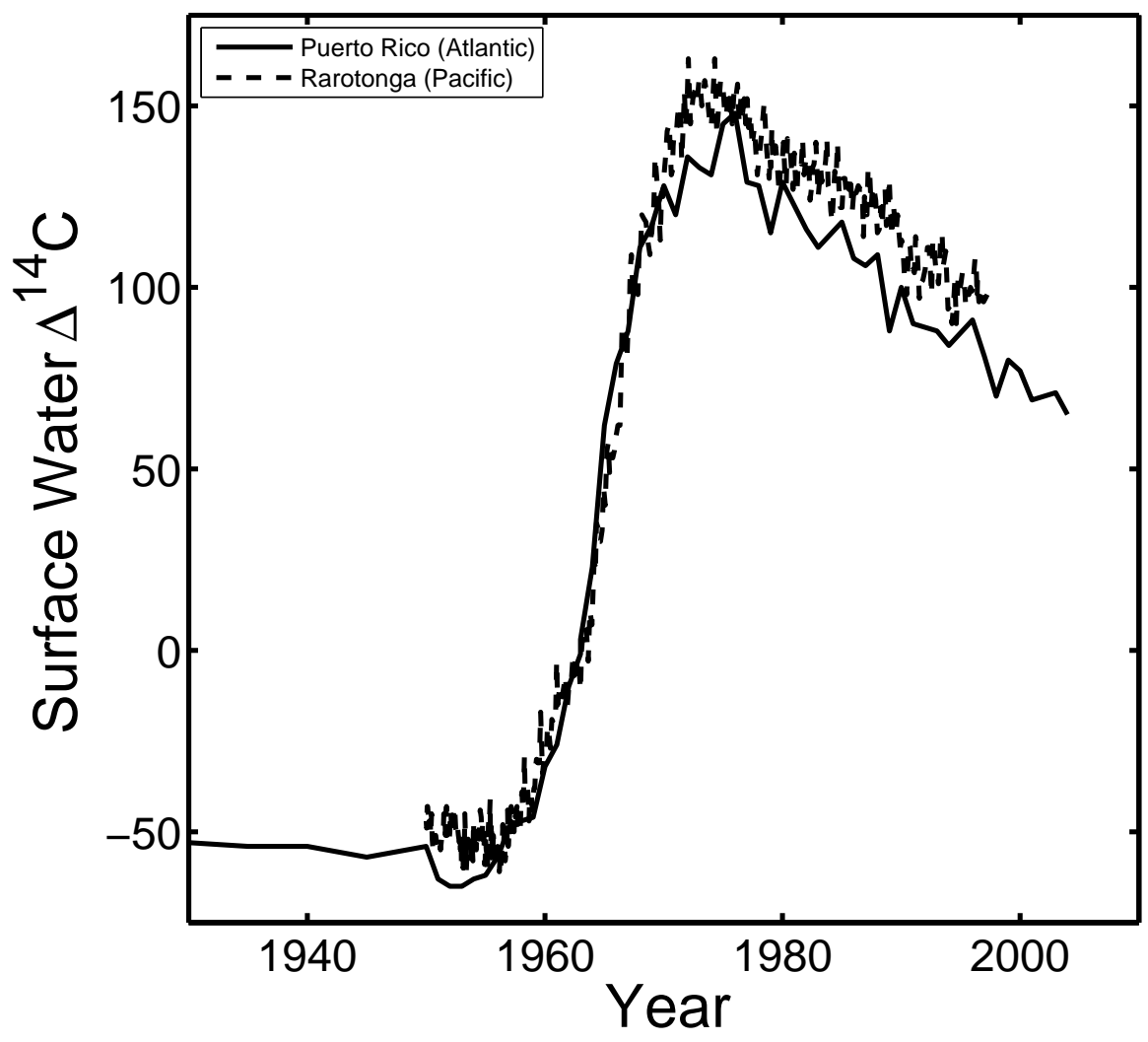

Figure 3-12: Two time-series of the estimated surface DI $\Delta^{14} \mathrm{C}$ spanning the injection of bomb radiocarbon into the surface ocean [19]. Data were from the edges of the gyres in the north Atlantic and south Pacific. Notice that both timeseries peak near 1975 and follow a linear downward trend for the remainder of the time-series. 
deep water concentration, then the estimated value for the excess component of DOC should never be outside the range of DI $\Delta^{14} \mathrm{C}$ inputs. However, if the deep component of DOC is itself a combination of background and excess carbon, then estimates outside of the known ranges are to be expected. For example, two measurements of DOC with nearly identical concentrations, but differing values of $\Delta^{14} \mathrm{C}_{x s}$ would yield a nearly vertical line in Keeling space with an intercept at either $\pm \infty$ independent of the true values of $\Delta^{14} \mathrm{C}_{x s}$. The prediction that the $\Delta^{14} \mathrm{C}_{x s}$ component increases from below surface DI $\Delta^{14} \mathrm{C}$ values at high concentration, to above the maximum seen surface DI $\Delta^{14} \mathrm{C}$ values at low concentration suggests that the deep water contains a significant portion of excess DOC with a radiocarbon value above the modern day surface.

For the Southern Ocean profile [47], multiple things stand out. There is a negative curvature with depth, and the estimate of the modern surface component based on the highest concentration data and the third cluster is over $250 \%$ despite the fact that surface ocean DI $\Delta^{14} \mathrm{C}$ at this site is closer to $10 \%$. A similar process as exists for positive curvature could exist here, but because of the negative curvature and the low surface DI $\Delta^{14} \mathrm{C}$ it seems unlikely that it is due to the nuclear bomb testing isotope spike. More likely is that the northward drift of deep water from areas with a low surface DI $\Delta{ }^{14} \mathrm{C}$ into areas with a higher $\mathrm{DI} \Delta^{14} \mathrm{C}$ provides a negative gradient of $\Delta^{14} \mathrm{C}_{x s}$ with increasing depth. In this case, we could explain both the negative Keeling curvature and the anomalously high values for the estimate of $\Delta^{14} \mathrm{C}_{x s}(150 \%$ while surface DI $\Delta^{14} \mathrm{C}$ is near $10 \%$ ). The extremely high drift rate of deep water in this region of near 1 degree per year suggests that deep water moves under a surface DI $\Delta{ }^{14} \mathrm{C}$ source which increases by $\sim 100 \%$ over the course of a decade. This would cause both a negative curvature in Keeling space and a $\Delta^{14} \mathrm{C}_{x s}$ estimate which is far too high. Both of these things occur.

Although the curvature seen in the North Central Pacific and Western North 
Pacific data is not significant, the estimate for $\Delta^{14} \mathrm{C}_{x s}$ in both locations is significantly higher than surface ocean DI $\Delta{ }^{14} \mathrm{C}[20]$. The linearity suggests that the depth profile equilibrates fast. This, along with the high $\Delta^{14} \mathrm{C}_{x s}$ estimate is consistent with the idea that bomb carbon DOC exists in the deep ocean. For the Western North Pacific, the situation is more confusing as the Keeling intercept is at least 100\% greater than the estimates of surface DI $\Delta{ }^{14} \mathrm{C}$. The original publishers of the data [48] suggest that perhaps this site is heavily influenced by terrestrial sources, yet a mechanism has not been elucidated. The influence of terrestrial sources is consistent with the $\delta^{13} \mathrm{C}$ values below $-23 \%$ for the entire depth profile [48].

Both the positive curvature and negative curvature discussed above could be caused by external inputs of carbon rather than the bomb spike and advection as suggested here. However, the nature of the curvature puts serious limitations on their dominance. Chemoautotrophic production of labile organic material at middepths in the water column [62] would cause negative curvature in Keeling space as labile carbon with a local DI $\Delta{ }^{14} \mathrm{C}$ value would be inserted into the DOC pool and increase the age of material relative to the true Keeling curve. This mechanism could yield the negative curvature we see in the Southern Ocean. However, a non-observed systematic shift in $\delta^{13} \mathrm{C}$ should accompany this curvature which is not observed [12]. Pre-aged material could also be preferentially injected at depth which would influence the curvature. This may be the case for the Eastern Pacific $[9,68]$ where a positive correlation between concentration and age was found for the deep ocean suggesting an external source of pre-aged material may be important at this site. Despite this, the propagation of the bomb spike at this location is strongly supported by the prediction of surface DOC radiocarbon below surface DI ${ }^{14} \mathrm{C}$. An 'artificial' decrease in the radiocarbon values of the deep ocean would cause an artificial increase in the estimate of $\Delta^{14} \mathrm{C}_{x s}$. If the bomb spike was not an issue, we would then expect the estimate of the Keeling intercept in the Eastern Pacific to be greater than DI $\Delta^{14} \mathrm{C}$. 


\subsubsection{Spatial Variability of Keeling Slope}

Re-analyzing the Keeling space plots for open ocean sites suggests that the deep, low concentration portion of DOC may contain a reasonable portion of relatively recent material from the surface. However, it is difficult to predict this purely from the Keeling plots themselves. Restricting ourselves further to open ocean sites where there is neither time-series based or isotopic evidence for non-autochthonous inputs to the DOC pool leaves us with three open ocean sites; the Sargasso Sea, the Southern Ocean, and the North Central Pacific. The lack of external inputs to these sites suggests that we can use the variability between them to put further constraints on the background component of the DOC.

By combining equations (3.28) and (3.29) (Supporting Information section 3.5) we can solve for the Keeling slope in terms of measurable variables and $\Delta^{14} C_{x s}$. Re-arranging equation (3.28) yields

$$
\left(\Delta^{14} C_{b k g} C_{b k g}-\Delta^{14} C_{x s} C_{b k g}\right)=\Delta{ }^{14} C_{m} C_{m}-\Delta{ }^{14} C_{x s} C_{m},
$$

or

$$
\Psi=\left(\Delta^{14} C_{m}-\Delta^{14} C_{x s}\right) C_{m} .
$$

We now assume that in the upper 100 meters, the radiocarbon value of the excess component, $\Delta^{14} C_{x s}$, is equal to DIC radiocarbon. If a constant background exists between the North Central Pacific, Southern Ocean and Sargasso Sea then a plot of $\Psi$ versus $\Delta^{14} C_{x s}$ should yield a straight line where the magnitude of the slope is the background concentration and the intercept divided by the slope is its radiocarbon value. For the three open ocean sites, Fig. 3-13, we estimate that the background concentration, $C_{b k g}$, is $20 \mu \mathrm{M}$ with a depleted radiocarbon value, $\Delta^{14} C_{b k g}$, of $-1000 \%$. We recognize the lack of data sites for this analysis, but note that this result is com-

pletely synergistic with the curvature found in the above Keeling plots. Additionally, 


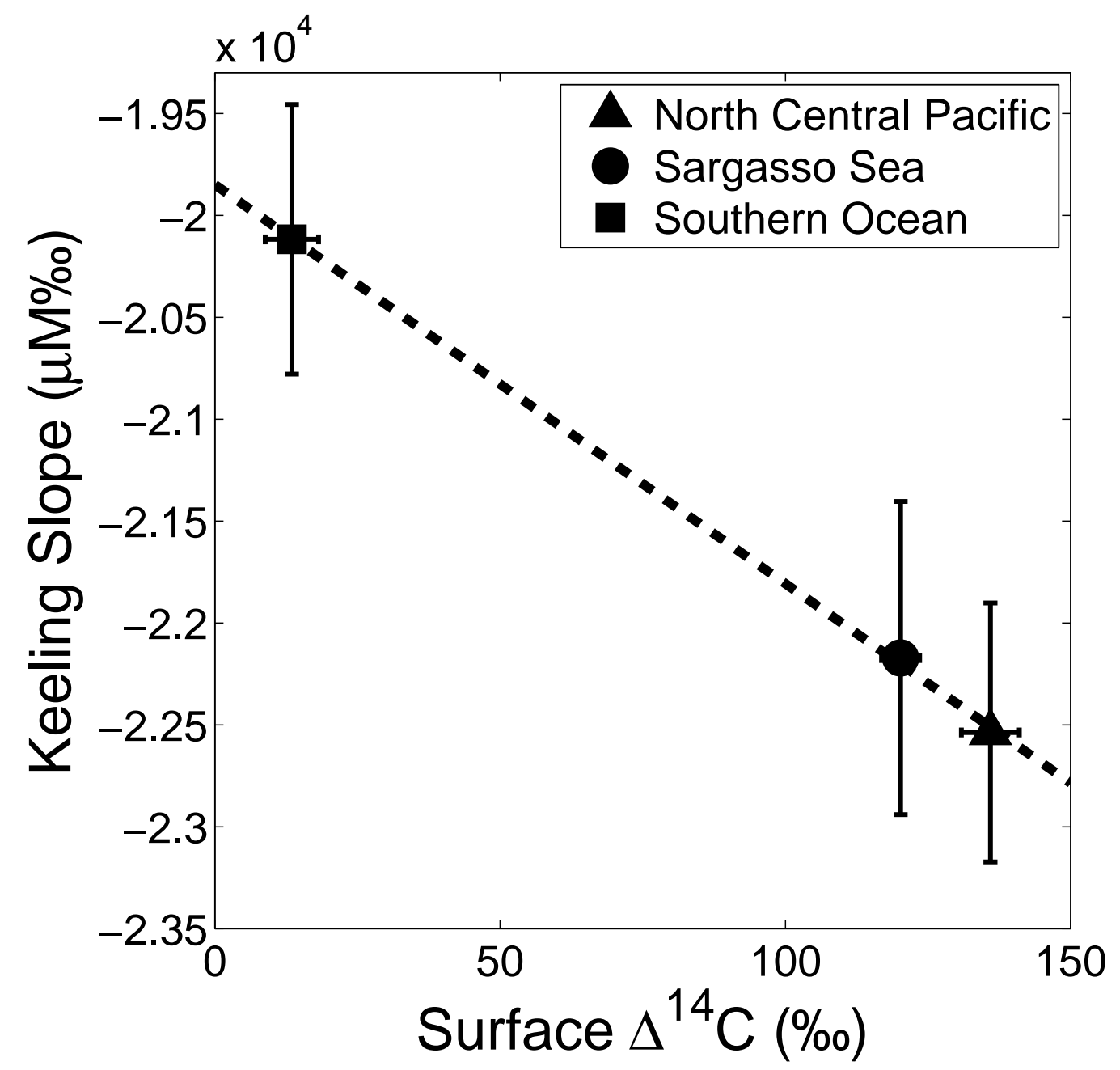

Figure 3-13: The mean Keeling slope, $\Psi$, is plotted versus the mean radiocarbon value of surface (1-100 meters) ocean DIC for the North Central Pacific Ocean [12], Sargasso Sea [12], and Southern Ocean [47]. Error bars represent \pm 1 standard error on the mean. The slope is $C_{b k g}=20 \mu \mathrm{M}$ for background DOC with an associated radiocarbon value of $\Delta^{14} C_{b k g}=-1000 \%$. 
if the background component is a subset of the deep ocean DOC, the mechanism exists to resolve one of the open questions in ocean DOC cycling: the anomalous aging of DOC in the Southern Ocean relative to DOC found in the deep Atlantic and North Central Pacific [47].

\subsubsection{Anomalous DOC Aging in the Southern Ocean}

If the average radiocarbon age of deep ( $>1000$ meter) water DIC is plotted versus the corresponding mean radiocarbon age for DOC, we would expect the data to lie on a line with a slope of unity. This slope can be straightforwardly understood in terms of the two-component model of DOC cycling. If deep water DOC is composed of material with a single, extremely long, turnover time, then there is no mechanism for the average age of the reservoir to change as the material is oxidized. Under these conditions, we would expect the DOC pool to age along with its corresponding DIC. If we plot this data for the North Central Pacific, Southern Ocean, and Sargasso Sea (Fig. 3-14) it is clear that the three sites do not lie on the correct aging line. Although the deep Sargasso Sea and North Central Pacific sites are consistent with conservative aging between the two sites, the Southern Ocean DOC appears to be 500-1000 years too old. Explaining this deviation requires that a second component of DOC exist in the deep ocean in at least one of these locations [47]. It is appealing to suggest that the anomalous aging of the deep DOC is due entirely to a preferential remineralization of younger components of the deep background pool of DOC relative to older ones. However, if this was the case, we would not expect deep water DOC to get relatively younger as it moved from the the Southern Ocean to the North Central Pacific. To accomplish this, there must be a secondary pool of carbon in the deep ocean. One possibility is that there is a small injection of extremely old, pre-aged material in

the Southern Ocean which is relatively labile. In this way, the mean age of the deep DOC in the Southern Ocean would be driven older and slowly get relatively younger 


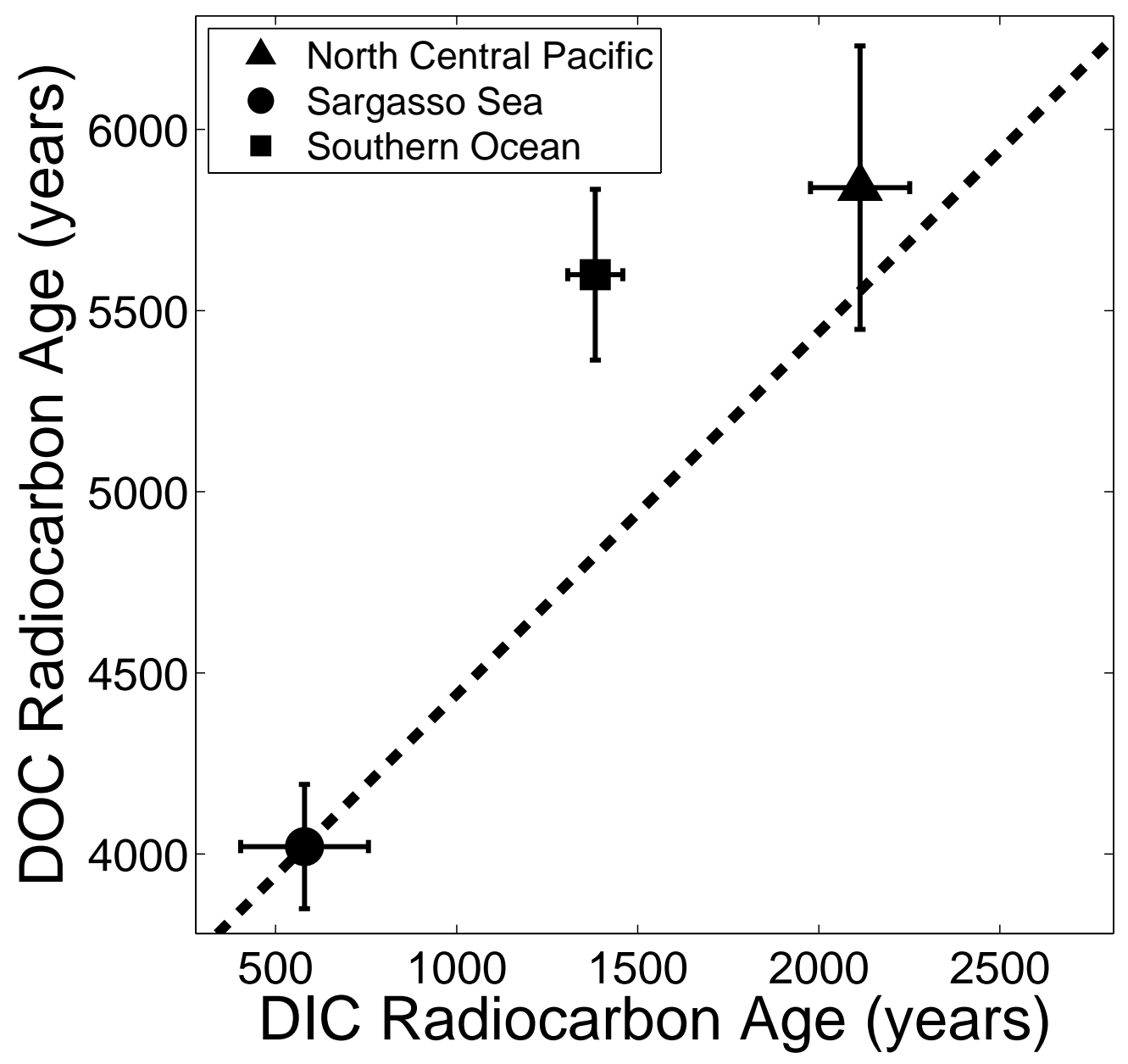

Figure 3-14: DOC radiocarbon age is plotted versus DIC radiocarbon age for the deep (> 1000 meters) North Central Pacific Ocean [12], Sargasso Sea [12], and Southern Ocean [47]. Mean values are plotted where missing DIC radiocarbon values were linearly interpolated from the depth profile. Error bars represent \pm 1 standard error on the mean. Points on the dotted line are consistent with conservative aging of both DOC and DIC. Age is given relative to the mean values of the Sargasso Sea. 
as the water moves north towards the North Central Pacific [47]. This mechanism would successfully explain the curvature of the Southern Ocean Keeling data and the high Keeling intercept when compared to ambient surface DI $\Delta^{14} \mathrm{C}$. However, no source which meets these criteria has been identified. Additionally, this mechanism requires other special inputs of carbon to explain the high Keeling intercept in the North Central Pacific, and the curvature of the Sargasso Sea data.

Allowing surface derived, semi-labile, carbon to penetrate the deep ocean DOC reservoir could mechanistically link all three of these sites and explain both their behavior in Keeling space and their deep ocean DOC radiocarbon values. Using the predicted value for the background carbon of $20 \mu \mathrm{M}$ and $-1000 \%$ we can plot the value of $\Delta{ }^{14} \mathrm{C}_{x s}$ for the surface $(<100 \mathrm{~m})$ as compared to the deep $(>1000 \mathrm{~m})$ water (Fig. 3-15). What we find is that the excess component relative to the deep excess component is consistent with both the Keeling plots at these sites and knowledge of the local oceanography. As discussed earlier, both the high Keeling intercept and negative Keeling curvature in the Southern Ocean are consistent with deep waters previously exposed to low surface DI $\Delta{ }^{14} \mathrm{C}$ values moving northward where higher surface DI $\Delta{ }^{14} \mathrm{C}$ values persist. This is seen in the comparison of $\Delta^{14} \mathrm{C}_{x s}$ for the surface and deep waters at this site. The Southern Ocean data plots above the 1:1 line with a $\Delta^{14} \mathrm{C}_{x s}$ in the surface near surface DI $\Delta{ }^{14} \mathrm{C}$ values and in the deep waters more depleted towards Southern Ocean surface DI $\Delta{ }^{14} \mathrm{C}$ values. At the Sargasso Sea and North Central Pacific sites, the data also plots as expected. The Sargasso Sea data plots below the 1:1 line consistent with the positive Keeling curvature at this site. The North Central Pacific data plots above the line, consistent with the high Keeling intercept. 


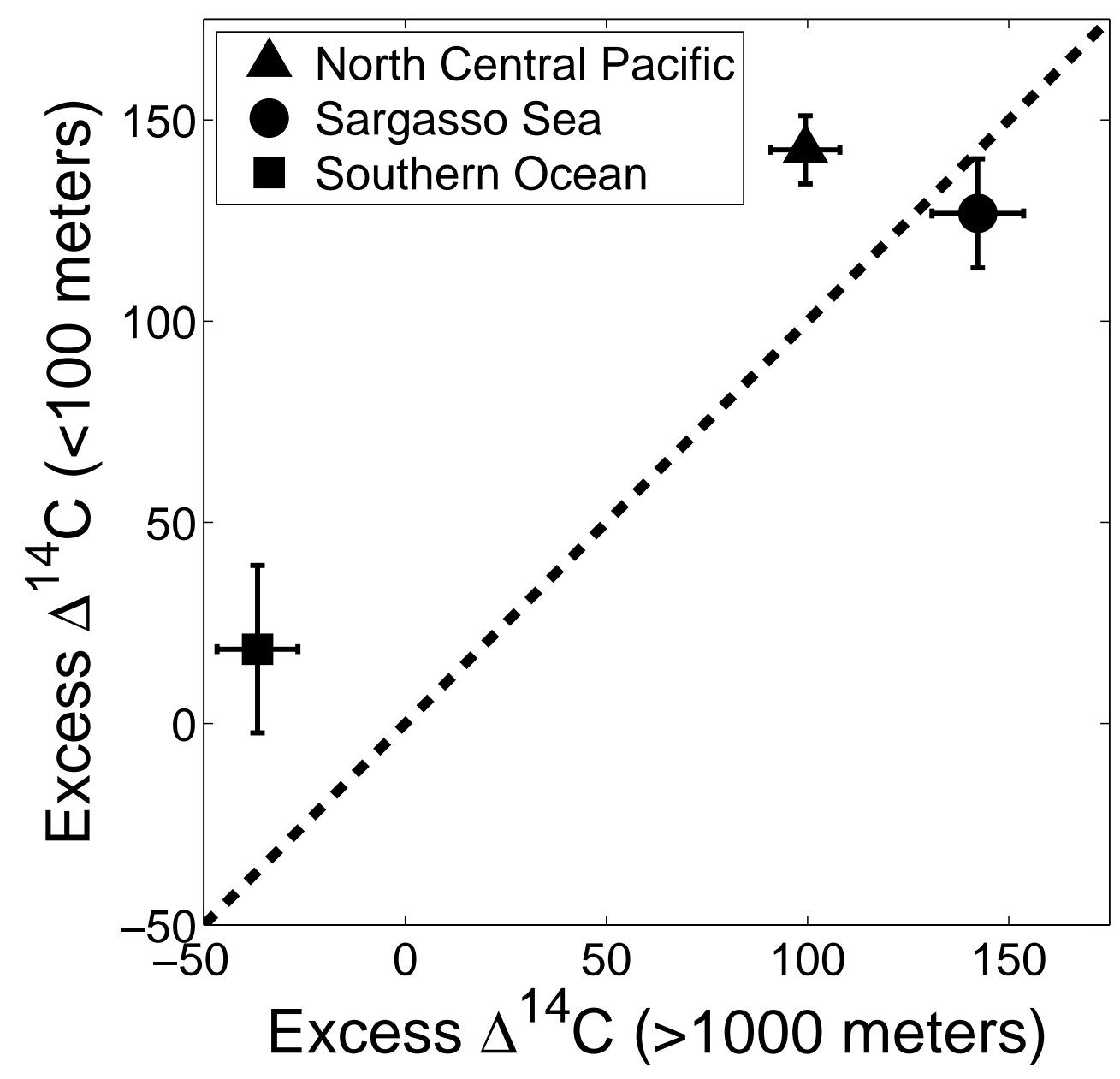

Figure 3-15: The mean excess carbon is plotted for surface and deep waters by subtracting the estimated values for the background component $(20 \mu \mathrm{M}$ and $-1000 \%)$ for the Sargasso Sea, Southern Ocean, and North Central Pacific data. Error bars represent \pm 1 standard error on the mean. Deviations from the 1:1 line (dashed line) are consistent with the curvature found in the Southern Ocean and Sargasso Sea Keeling plots as well as the high Keeling intercept [20] of the North Central Pacific Data. 


\subsubsection{Direct Estimation of Equilibration Timescale from Bomb- spike Information}

We can take this analysis one step further and estimate the equilibration timescale for the deep water excess component in the Sargasso Sea and North Central Pacific. As

the flow of deep water dominates the signal in the Southern Ocean, it is unfortunately not possible to do this analysis at that site. If we assume that the excess component in the deep ocean is in a first order steady state with surface DI $\Delta^{14} \mathrm{C}$, then we can write the $\Delta{ }^{14} \mathrm{C}_{x s}$ value in terms of the surface ocean DI $\Delta{ }^{14} \mathrm{C}$ and the turnover time $\tau$ for that reservoir as

$$
\Delta^{14} \mathrm{C}_{x s}=\int_{-\infty}^{0} \frac{D_{s}}{\tau} e^{t / \tau} d t,
$$

where $D_{s}$ is the surface DI $\Delta^{14} \mathrm{C}$ as a function of time. The seemingly positive term in the exponential is due to the negative limits of integration. We can further simplify things by approximating the surface DI $\Delta{ }^{14} \mathrm{C}$ time series as a step function equal to a pre-bomb value $D_{b}$ until a time, $t_{c}$, and a post bomb value $D_{a}$ for the remainder of time. Substituting into equation (3.19) then yields

$$
\Delta^{14} \mathrm{C}_{x s}=\int_{-\infty}^{t_{c}} \frac{D_{b}}{\tau} e^{t / \tau} d t+\int_{t_{c}}^{0} \frac{D_{b}}{\tau} e^{t / \tau} d t
$$

or

$$
\Delta^{14} \mathrm{C}_{x s}=D_{a}+\left(D_{b}-D_{a}\right) e^{t_{c} / \tau} .
$$

We can then solve this for the equilibration timescale

$$
\tau=t_{c}\left(\ln \left(\frac{\Delta^{14} C_{x s}-D_{a}}{D_{b}-D_{a}}\right)\right)^{-1} .
$$

Setting the critical date to 1970, ( $t c$ will equal 1970 minus the observation date), $D_{b}=-50 \%, D_{a}=150 \%$, and getting $\Delta{ }^{14} \mathrm{C}_{x s}$ from the previous analysis yields a 


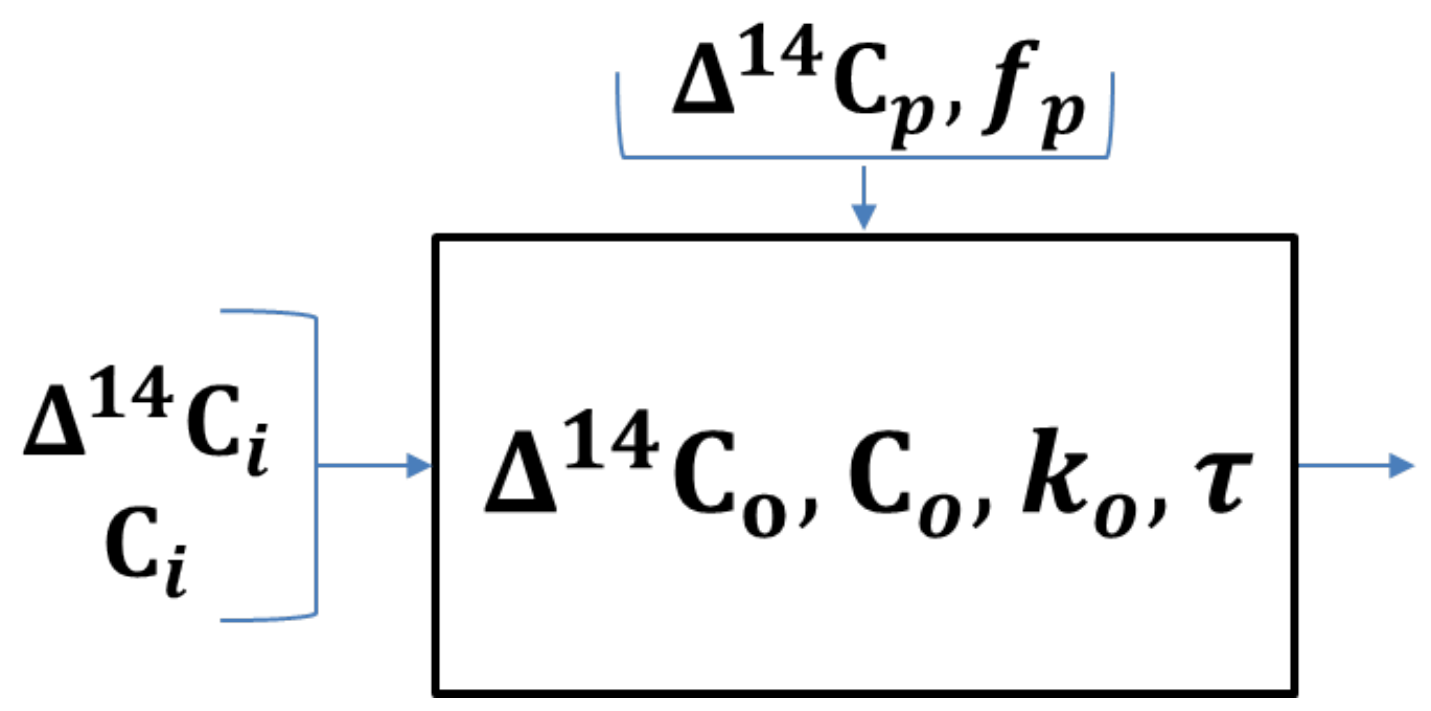

Figure 3-16: The feasibility of semi-labile replacement as the driver for DOC 'ageing' is tested with a simple box model. Inputs originating from the Atlantic Ocean (subscript $i$ ) combine with local particle inputs, $p$, to yield the local values $o$ as water travels between ocean basins with timescale $\tau$. Subscripts, $(1,2,3)$, denote semi-labile, recalcitrant, and the total carbon reservoir respectively.

timescale $\tau \approx 11$ years for the North Central Pacific Site and $\tau \approx 6.5$ years for the Sargasso Sea site. The longer timescale at the Pacific as compared to the Atlantic site is consistent with the measured two times the particle flux at the Atlantic versus the Pacific Ocean site [12]. Both of these timescales are fast and strongly influenced by the potentially low estimate for $D_{a}$ and the extremely low value of $\Delta^{14} C_{b k g}$.

\subsubsection{Constraining Deep Water DOC with a Simple Box Model}

In order to further explore the parameter space of background components in the deep ocean we turn to a simple two-component box model (Fig. 3-16) which accounts for the aging of the background component as it travels with deep waters from the North Atlantic to the North Pacific oceans. We can express this model as a simple set of first order differential equations:

$$
\frac{d C_{o}}{d t}=-k_{o} C_{o}-\frac{1}{\tau} C_{o}+\frac{1}{\tau} C_{i}+f_{p}
$$


and

$$
\frac{d R_{o} C_{o}}{d t}=-k_{o} R_{o} C_{o}-\frac{1}{\tau} R_{o} C_{o}+\frac{1}{\tau} R_{i} C_{i}+R_{p} f_{p}-\lambda R_{o} C_{o}
$$

where equation (3.26) is the equation for concentration and 3.27 is the equation for radiocarbon. Here, the subscript $o$ refers to the value at the second site (Southern Ocean, or North Central Pacific) for either the labile, $o=1$, or recalcitrant background $o=2$ components. The subscripts $p$ and $i$ take on the same values and refer to the surface derived inputs due to particle fluxes $(p)$ or the advective inputs from the Atlantic $(i)$. Radiocarbon values are expressed by

$$
R=\frac{\Delta^{14} C+1000}{1000}
$$

while $\lambda$ is the radioactive decay constant for ${ }^{14} \mathrm{C}$. Decay is governed by both the first order decay constant, $k_{o}$, and by the transit timescale, $\tau$.

Taking count of all the subscripts, we have four differential equations, two for the labile deep component and two for the recalcitrant deep component, yet we have eleven unknowns. Assuming we have a steady state gives us four algebraic equations. Using the known total mass reduces the unknowns to 9 . We then assume that the recalcitrant fraction does not decay, and that the turnover time for the deep excess component is much smaller than the transit time $\tau$. Under these additional assumptions, knowledge of the concentration of the background component of DOC and the radiocarbon value of the excess component in the deep Atlantic allow you to solve for the radiocarbon value of the excess component at the second site and the initial radiocarbon value of the background carbon. Scanning a concentration range of $0-35 \mu \mathrm{M}$ for $C_{b k g}$, a $\Delta^{14} C_{x s}$ range of -50 to $150 \%$ for the Atlantic site, and constraining the $\Delta^{14} C_{x s}$, and $\Delta^{14} C_{b k g}$ values to be consistent with observation yield the feasibility maps in figures 3-17 and 3-18. These maps can be re-projected in axes of $C_{b k g}$ versus $\Delta^{14} C_{b k g}$ at the Atlantic site where overlapping regions represent feasible values for 


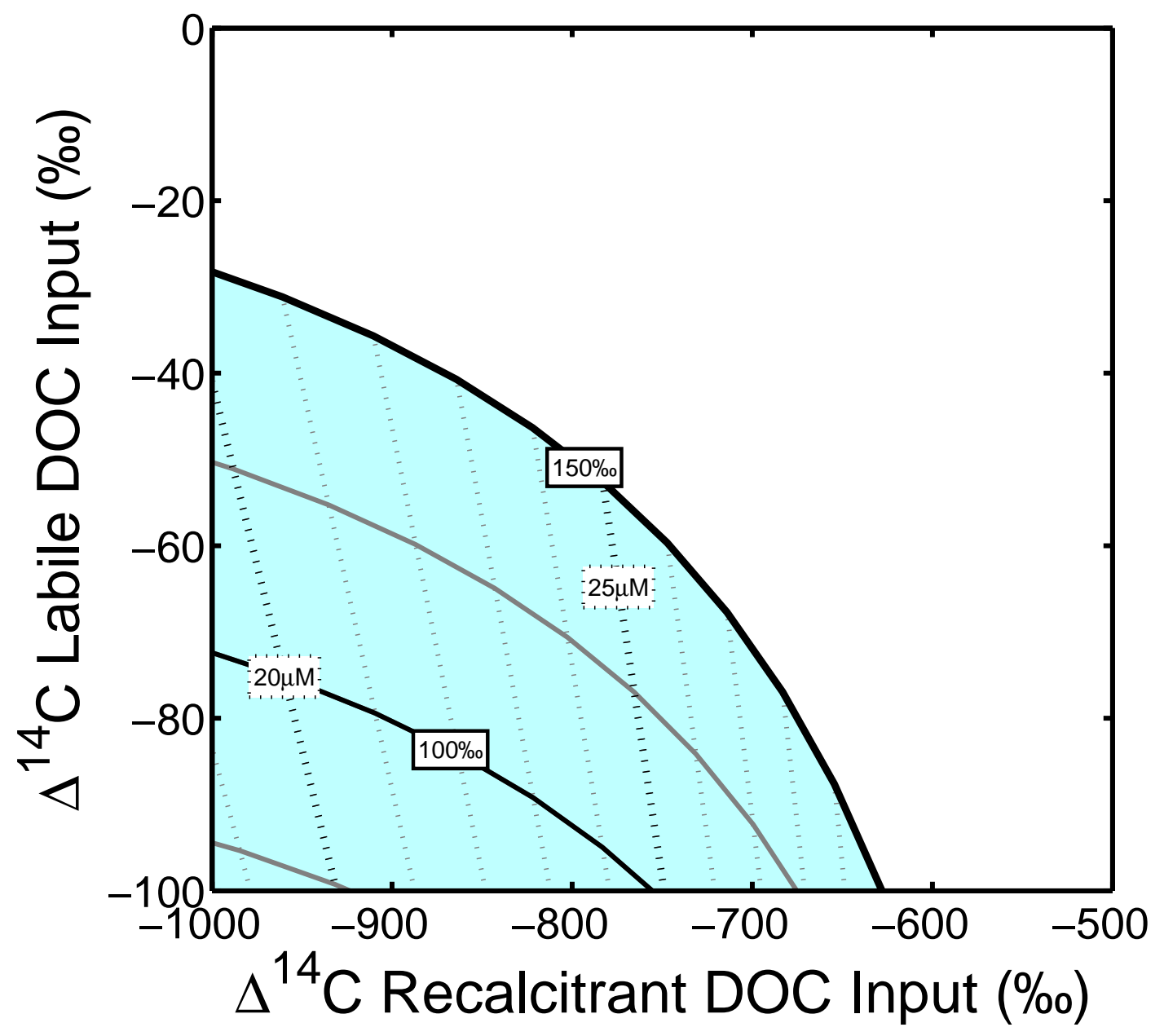

Figure 3-17: This is the region of feasibility for the Southern ocean based on the simple box model described in the text. Labile DOC inputs were constrained to be greater than $-100 \%$ and the background component greater than $-1000 \%$. The concentration of the background component (vertical dashed lines) ranged from 1 $35 \mu \mathrm{M}$ and the initial $\Delta^{14} C_{x s}$ (solid curves) was kept between -50 and $150 \%$. 


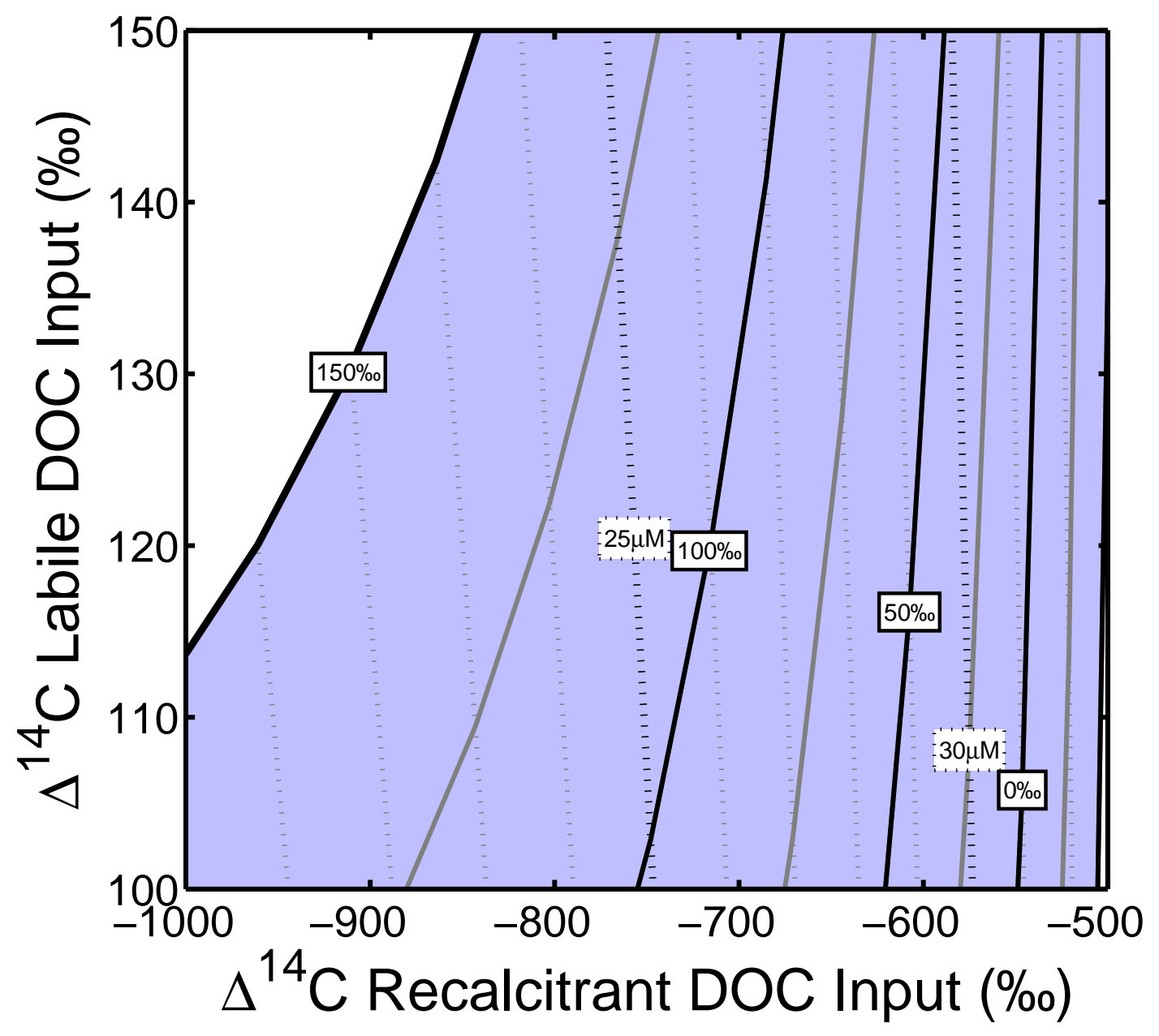

Figure 3-18: This is the region of feasibility for the North Central Pacific ocean based on the simple box model described in the text. Labile DOC inputs were constrained to be greater than $-100 \%$ and the background component greater than $-1000 \%$. The concentration of the background component (vertical dashed lines) ranged from $1-35 \mu \mathrm{M}$ and the initial $\Delta^{14} C_{x s}$ (solid curves) was kept between -50 and $150 \%$. 
the background component of DOC. According to the overlapping region in figure 3-19 a background component of constant concentration could have a minimal value of $\Delta^{14} C_{b k g} \approx-1000 \%, C_{b k g} \approx 20 \mu \mathrm{M}$ and a maximal value near $\Delta{ }^{14} C_{b k g} \approx-700 \%$, $C_{b k g} \approx 27 \mu \mathrm{M}$.

\subsection{Final Thoughts}

Together, these analyses provide evidence for the presence of excess DOC in the deep ocean cycling on fast timescales relative to deep ocean circulation. While at first this view seems contradictory to the TCM, it has been suggested on multiple occasions $[8,10]$ and is an extension of current thought. We simply believe that the excess component of DOC is not completely gone in the deep ocean.

In addition to the analyses done here, addition of particulate material to the deep DOC reservoir is consistent with an array of external observations. With the recognition of substantial microbial metabolism in the deep ocean [69], a buffering reservoir of carbon would be expected as microbes feed entirely through the uptake of dissolved species. Additionally, the protein species RUBISCO, a photosynthetic enzyme, has been found in the deep ocean at quantities close to $.5 \mu \mathrm{M}$, with the largest concentrations in equatorial regions [70]. Although possible that this enzyme is awarded special protection in the deep sea, laboratory experiments suggest a half life on the order of days [71]. This turnover time should be decreased by both low concentrations and temperatures, but should be smaller than a thousand year timescale. This observation and the fluctuations in concentration are consistent with a large excess reservoir of DOC in the deep ocean.

Although modeled as a simple mixture of two components, [16], the majority of DOC radiocarbon data is explained with the help of particle inputs (data excluded from some of our above analysis) or other external sources $[9,46,48,64]$. If these cites 


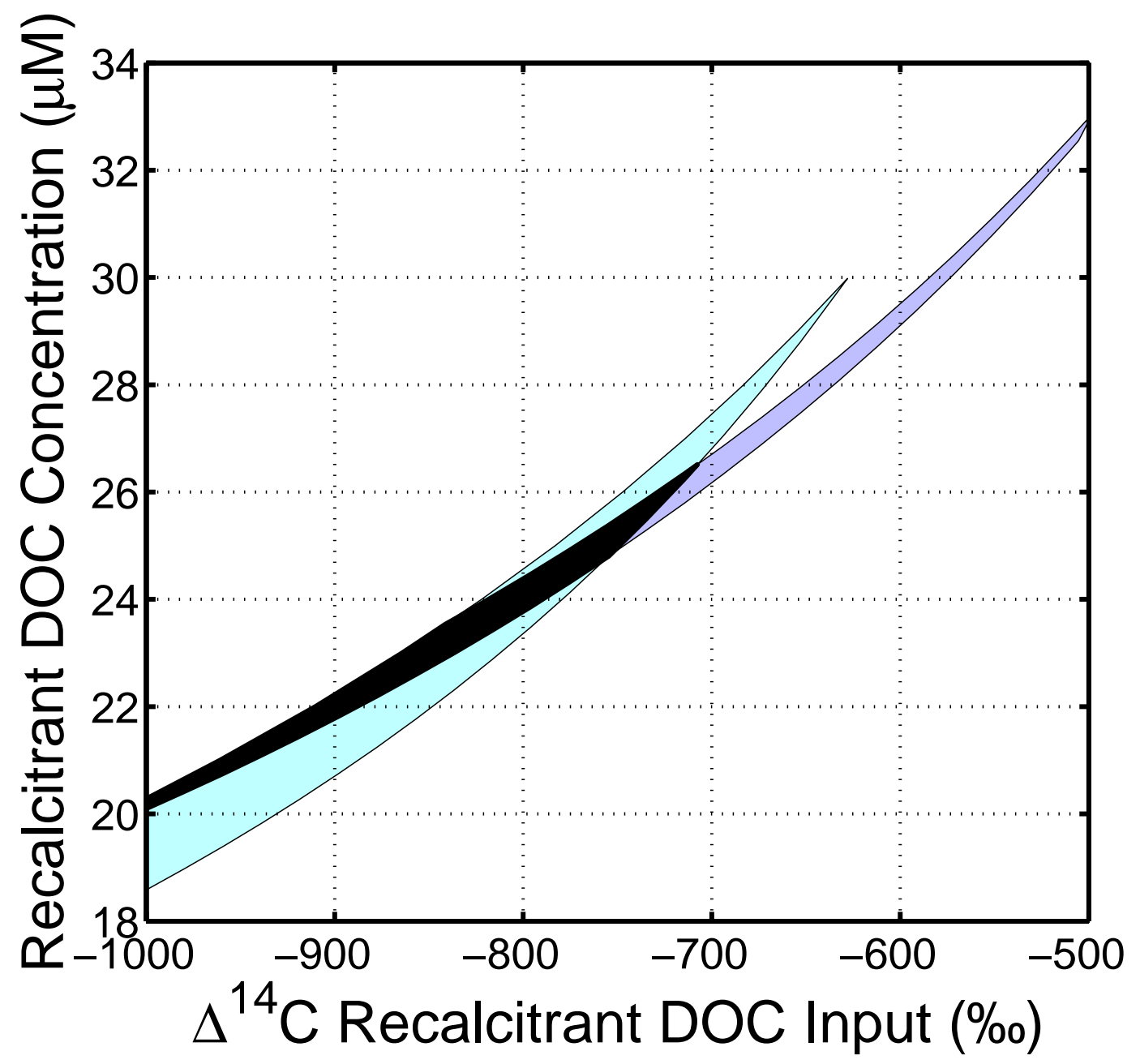

Figure 3-19: The regions of feasibility for the Southern Ocean (blue region) and North Central Pacific Ocean (purple region) are plotted where the overlapping region (black) is feasible values for the background component of DOC in the Atlantic. Note that this region is both lower in concentration and in radiocarbon value than anything observed in bulk measurements and that the value predicted from surface waters is contained in the feasible region. 
near sources of terrestrial particulate carbon feel its influence then it is not a large step to believe that particle fluxes in the open ocean may be felt by the deep DOC reservoir.

The idea that the deep background component of organic carbon may be overlayed by a modern portion is by no means new $[8,10]$ and is conceptually appealing given the heterogeneous nature of this reservoir of carbon and its many potential sources and sinks. The analysis done here suggests that the radiocarbon modern reservoir of carbon in the deep ocean may equilibrate with surface carbon on observable timescales with the potential for measurement. For example, if the positive curvature we find in the Sargasso Sea [12] Keeling plots is due to deep equilibration with the bomb spike, then a re-occupation of this site should yield measurable changes in the radiocarbon value of deep water DOC there. This might be especially true in the deep North Central Pacific where linearity suggests that equilibration is fast, yet the slope and intercept are too high [20]. As surface ocean DOC at that site decreased over the last 30 years we would expect that the values of the deep ocean DOC there have increased. Undeniable evidence for a fast equilibrating reservoir of carbon in the deep ocean might come from a deep horizontal transect where the DI $\Delta{ }^{14} \mathrm{C}$ values of the deep water decreased while the surface DI $\Delta{ }^{14} \mathrm{C}$ values increased. A deep water transect coming out of the Southern Ocean into the Western South Pacific might satisfy all of these criteria. Under this situation the surface DI $\Delta^{14} \mathrm{C}$ values change by as much as $250 \%$ yielding enough resolution to overcome the $\pm 20 \%$ variability in radiocarbon measurements on seawater.

For most of the last thirty years, DOC in the deep ocean has been considered to be in a very slow decay cycle lasting thousands of years. Although the presence of radiocarbon depleted carbon in the deep DOC reservoir is clear, whether the entire reservoir is biologically isolated is less obvious. By allowing a substantial reservoir of semi-labile excess carbon to exist in the deep ocean, one can explain both the 
curvature and the extreme Keeling estimates of excess DOC as a function of depth or decreasing bulk concentration. Additionally, the anomalous aging of deep DOC between the Atlantic Ocean, Southern Ocean, and Pacific Oceans can be resolved naturally in terms of variable inputs and the TCM. This has the added benefit of being consistent with surface DOC and DOC radiocarbon measurements at these sites and with a simple box model. Perhaps deep ocean DOC is not truly isolated, but exists in more of a steady-state, consistent with the heterogeneous processes affecting it.

\subsection{Supporting Information}

\subsubsection{Derivation of Keeling Line}

Assuming a two component system, any measurement of DOC concentration and radiocarbon value is expressible as a sum of its component parts. Conservation of mass then yields

$$
C_{m}=C_{x s}+C_{b k g}
$$

for concentration, and

$$
\Delta^{14} C_{m} * C_{m}=\Delta{ }^{14} C_{x s} * C_{x s}+\Delta{ }^{14} C_{b k g} * C_{b k g}
$$

for radiocarbon, where the subscripts $m, x s$, and $b k g$ refer to the measured, excess, and background components of DOC. This can be reduced to the Keeling form,

$$
\Delta^{14} C_{m}=\left(\Delta^{14} C_{b k g} C_{b k g}-\Delta^{14} C_{x s} C_{b k g}\right) \frac{1}{C_{m}}+\Delta^{14} C_{x s},
$$

where a plot of $\Delta^{14} C_{m}$, versus $1 / C_{m}$ yields a straight line and $\Delta^{14} C_{x s}$ is the intercept with the vertical axis. To aid in the discussion we denote the slope of lines in Keeling 
space by

$$
\Psi=C_{b k g}\left(\Delta^{14} C_{b k g}-\Delta^{14} C_{x s}\right)
$$

\subsubsection{Example of Keeling Errors Affecting the Mean}

When fitting linear data in a non-linear space, like Keeling space, errors which are originally uncorrelated with the axis values can become a function of either the horizontal or vertical axes. In the standard fitting approach for Keeling space, linear errors are propagated into the estimate of $\Delta^{14} C_{x s}$. This can be straightforwardly demonstrated through simulation. We generate a fake depth profile using equation (3.28) and set $\Delta^{14} C_{x s}=100, \Delta^{14} C_{b k g}=-1000$, and $C_{b k g}=20 \mu \mathrm{M}$. We use a concentration range of $70-35 \mu \mathrm{M}$ and evenly space the data in $1 / C_{m}$. We then estimate $\Delta^{14} C_{x s}$ by fitting a straight line in Keeling space and best fit line as a function of the error in $\Delta^{14} C_{m}$ for 100,000 data points evenly spaced in inverse concentration before random errors are applied (Fig. 3-20). Comparing the two approaches suggests that clustering eliminates the systematic dependence of the $\Delta^{14} C_{x s}$ estimate with increasing mass errors. 


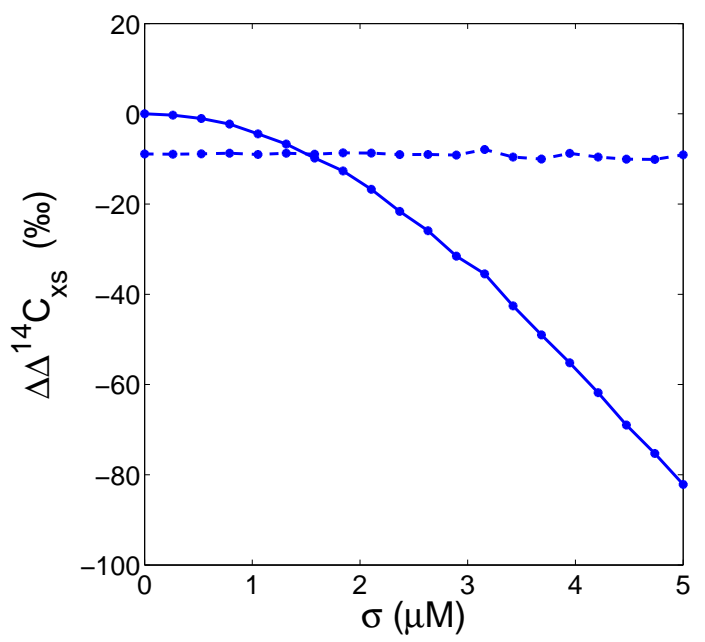

Figure 3-20: A plot of the deviation between the measured and prescribed values of $\Delta^{14} C_{x s}$ versus the standard deviation $(\sigma)$ in the measured concentration values in the simulation. The results of fitting a straight line in Keeling space (solid connecting curve) are plotted with the results of two component cluster analysis (broken curve). Notice that although cluster analysis seems to always underestimate $\Delta^{14} C_{x s}$ by around $10 \%$, the deviation is independent of $\sigma$ whereas the linear fitting approach shows severe error dependence. 


\section{Chapter 4}

\section{Hidden Cycle of Dissolved Organic Carbon in the Deep Ocean}

Marine dissolved organic carbon (DOC) is a large (660 Pg C), reactive carbon reservoir that mediates the oceanic microbial food web, and interacts with climate on both short and long timescales. Carbon isotopic content provides information on the DOC source via $\delta^{13} \mathrm{C}$ and age via $\Delta^{14} \mathrm{C}$. Bulk isotope measurements suggest a microbially sourced DOC reservoir with two distinct components of differing radiocarbon age. However, such measurements cannot distinguish internal dynamics and fluxes. Here we analyze serial oxidation experiments to quantify the isotopic diversity of DOC at an oligotrophic site in the central Pacific Ocean. Our results show diversity in both stable and radio isotopes at all depths, uncovering DOC cycling hidden within bulk analyses. We confirm the presence of isotopically enriched, modern DOC cocycling with an isotopically depleted older fraction in the upper ocean. However, our results show that up to $30 \%$ of the deep DOC reservoir is modern and supported by a $1 \mathrm{Pg}$ per year carbon flux, ten times higher than inferred from bulk isotope measurements. Isotopically depleted material turns over at an apparent time scale of 30, 000 years, far slower than indicated by bulk isotope measurements. These results are consistent with global DOC measurements and explain both the fluctuations in deep DOC concentration and the anomalous radiocarbon values of DOC in the Southern Ocean. Collectively these results provide an unprecedented view of the ways in which DOC moves through the marine carbon cycle. 


\subsection{Introduction}

Radiocarbon is a natural tracer of carbon flow through DOC [72]. As plankton grow and are consumed by grazers, organic matter with a modern radiocarbon value $\left(\Delta^{14} \mathrm{C}>-50 \%\right)$ is released into surface waters where it accumulates as "semi-labile" DOC $[8,12,73]$. Semi-labile DOC undergoes net remineralization below the euphotic zone, and gradually diminishes in concentration with depth to approximately $1000 \mathrm{~m}$, below which it appears to vanish. Oceanic profiles of total DOC and DOC radiocarbon (DOC $\Delta^{14} \mathrm{C}$ ) are therefore characterized by high values (60 to $80 \mu \mathrm{M}$ carbon; -200 to $-400 \%$ ) in surface waters and lower values at depth (35 to $40 \mu \mathrm{M} ;-400$ to $-550 \%$ ) [12]. The -200 to $-300 \%$ depletion in DOC $\Delta{ }^{14} \mathrm{C}$ values in surface seawater relative to semi-labile DOC $\Delta^{14} \mathrm{C}$ indicates the presence of a second refractory DOC fraction with an old radiocarbon age. The inverse proportionality between DOC concentration and radiocarbon value in depth profiles suggest that refractory DOC is well mixed throughout the entire water column $[8,10,12,20]$. The origin of the refractory DOC fraction is obscure, but stable isotopes $\left(\delta^{13} \mathrm{C}\right)$ show little change with depth, $\delta^{13} \mathrm{C}=-21.7 \%$ ( -23.2 to $-20.2 \%$ ) $[9,12,48,74]$, suggesting a common, autochthonous planktonic source for both fractions [12]. DOC and DOC $\Delta^{14} \mathrm{C}$ profiles can be reproduced in a simple two-component model (TCM) that includes a variable amount of semi-labile DOC cycling in the upper ocean $(<1000 \mathrm{~m})$ superimposed on a constant background of radiocarbon depleted, refractory DOC $[8,12]$. In the TCM, semi-labile DOC cycles on timescales of months to years, while refractory DOC cycles over several millennia $[8,12,51]$. The TCM provides an excellent one dimensional representation of DOC and isotope values in seawater, suggesting that microbial transformations of planktonic organic matter drive the marine DOC cycle.

The simple mixing of two isotopically distinct components implicit in the TCM can be contrasted with the wide range of mass fluxes and isotope values measured in potential sources of marine DOC. These include terrestrial organic matter from 
$\mathrm{C}_{3}$ and $\mathrm{C}_{4}$ plants delivered by rivers $[72,73,75]$, chemosynthetic organic matter from hydrothermal vent systems [76], organic matter derived from the oxidation of sedimentary methane [77], atmospheric deposition of black carbon from fossil fuel and biomass burning [78,79], chemoautotrophy in the mesopelagic zone [62], and organic matter released from sinking particles $[15,68,80]$. Together these sources represent a $\delta^{13} \mathrm{C}$ range of -14 to $-43 \%$, a $\Delta^{14} \mathrm{C}$ range of 150 to $-1000 \%$, and a carbon flux of over $3.5 \mathrm{Pg}$ per year. It seems unlikely that all of these sources are highly labile and non-accumulating. Compound and class specific isotope analyses have shown diversity within a very small fraction $(1-2 \%)$ of DOC $[81,82]$, but have not been able to connect specific isotope values to a corresponding inventory or mass flux. The discrepancy between the isotopic diversity in DOC sources and the narrow isotopic range of bulk DOC measurements has two resolutions. Either significant cycling of DOC is hidden by the TCM or many of these sources do not accumulate within the marine DOC reservoir.

\subsection{The Isotopic Distribution within DOC}

To estimate the isotopic distributions of components within DOC and quantify carbon fluxes that may be obscured by the TCM, we analyzed the step-wise oxidation of DOC under high-intensity ultraviolet light [23,43]. Samples were collected in July, 2010 from Station ALOHA (22 $\left.2^{\circ} 5^{\prime} \mathrm{N} ; 158^{\circ} \mathrm{W}\right)$, site of the Hawaii Ocean Time-series (HOT) program. Whole seawater was frozen and returned to the National Ocean Sciences Accelerator Mass Spectrometer facility in Woods Hole, MA (USA) for processing. Step-wise oxidation was performed using radiocarbon clean procedures on a customized, large volume UV apparatus. Oxidation under ultraviolet light was used, not as an analog of environmental processes, but as a tool to isotopically characterize DOC based on its oxidation rates. The total mass and mean isotopic value of DOC 

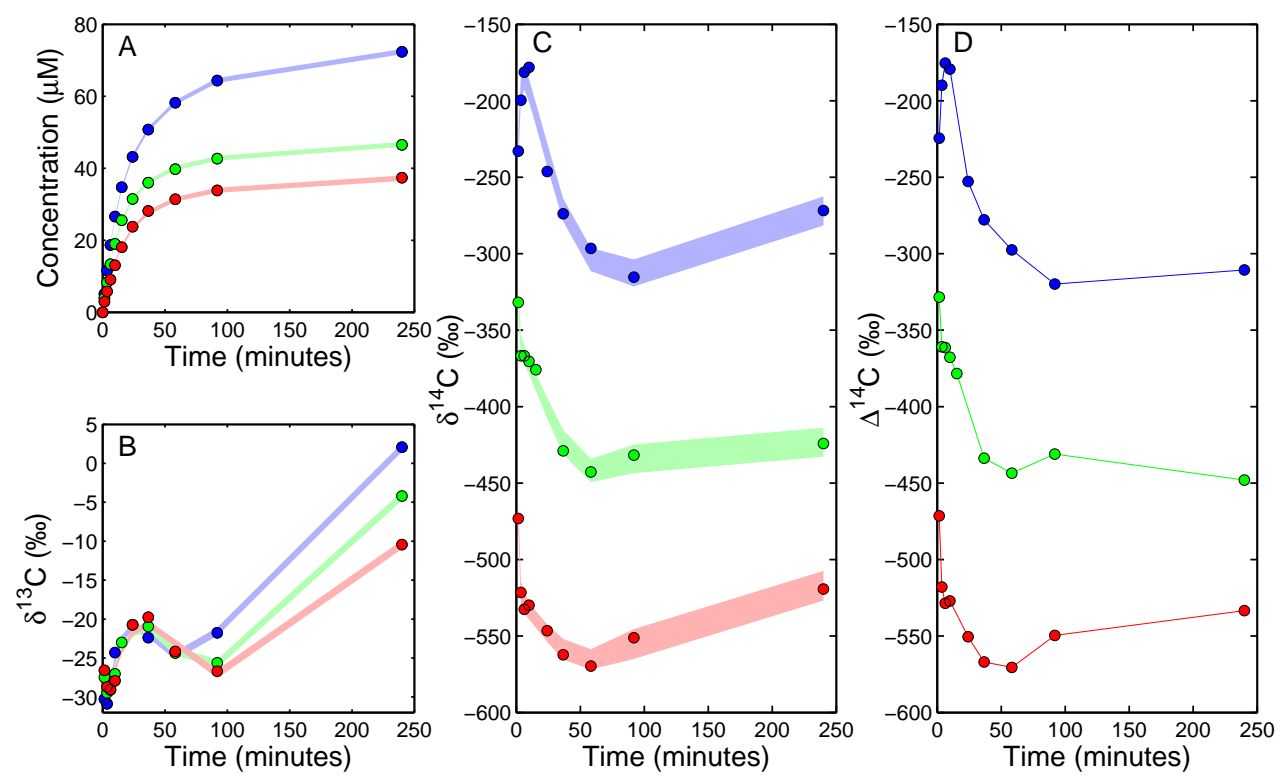

Figure 4-1: Oxidation data and fit: Time series showing cumulative mass per liter of seawater (A), local $\delta^{14} \mathrm{C}(\mathrm{B})$, local $\delta^{13} \mathrm{C}$ isotope values $(\mathrm{C})$, and local $\Delta^{14} \mathrm{C}$ values of carbon dioxide generated during step-wise UV oxidation of Station ALOHA DOC. Samples are from $50 \mathrm{~m}$ (blue), $500 \mathrm{~m}$ (green), and $2000 \mathrm{~m}$ (red) depths. The $95 \%$ confidence intervals for our 6 component fit are shown by the shaded regions. The connecting line for the $\Delta^{14} \mathrm{C}$ data is a guide for the eye, as this data is not suitable for our fitting procedure.

in surface, mid-depth, and deep seawater agrees with earlier results from a remote site in the North Pacific Subtropical Gyre (Supporting Table 1) used to establish the TCM $[8,12]$. The $100 \%$ depletion in our surface water $\Delta^{14} \mathrm{C}$ value, relative to those measured two decades ago, is due to the location of station ALOHA on the southern flank of the NPSG, and the slow removal of bomb radiocarbon (radiocarbon produced during atmospheric testing of nuclear weapons) from the surface ocean over the last two decades [83].

Figure 4-1 shows the serial oxidation time-series for our samples (numeric values in Supporting Table 1), and includes concentration, $\delta^{13} \mathrm{C}$, and $\delta^{14} \mathrm{C}$. We use $\delta^{14} \mathrm{C}$ in our analysis rather than $\Delta^{14} \mathrm{C}$ because our methods require linear isotope units. It should however be noted that the $\Delta^{14} \mathrm{C}$ and the $\delta^{14} \mathrm{C}$ time-series are similar, with 
the only substantial deviation being the final time point. $\Delta^{14} \mathrm{C}$ was calculated using the $\delta^{14} \mathrm{C}$ and separately measured $\delta^{13} \mathrm{C}$ values. Time points were chosen to minimize mass differences between each fraction to equalize statistical errors, and keep mass dependant errors systematic [23]. Mass values were blank corrected from a separate time series on previously oxidized seawater.

The structure of the isotope time-series provides direct information on the isotopic composition of DOC. The $\delta^{13} \mathrm{C}$ time series has two local minima at all three depths suggesting at least three distinct $\delta^{13} \mathrm{C}$ fractions. One minima occurs after approximately five minutes of total oxidation time (see data in Supporting Table 1 to resolve early times), while the other occurs after nearly 90 minutes. As the final $\delta^{13} \mathrm{C}$ value at all three depths is extremely enriched, fractionation must play a role in this system with measured fractionation factors for small organic compounds being as great as $10 \%$ [24]. Even with this fractionation, the fluctuation in isotope values suggest a spread of at least $10 \%$ for $\delta^{13} \mathrm{C}$ at all three depths. Fractionation is less relevant for $\delta^{14} \mathrm{C}$ as the dynamic range is ten times larger than for $\delta^{13} \mathrm{C}$. For the $\delta^{14} \mathrm{C}$ time series, the local extremal values, along with the decreasing trend also suggest three isotopic groups with a minimal spread of $150 \%, 120 \%$, and $100 \%$ for the surface, middle, and deep water respectively. However, the large changes in isotopic values, especially after long oxidation times, suggest DOC components with a much larger isotopic range. To estimate the full isotopic range we require a kinetic model for oxidation under ultraviolet light.

The oxidation of DOC by ultraviolet light has been modeled as both parallel first order $[23,24]$ and second order kinetic process [43] where the DOC concentration monotonically decreases while both the $\delta^{13} \mathrm{C}$ and $\delta^{14} \mathrm{C}$ values of the oxidation product (carbon dioxide) are free to fluctuate (Fig. 4-1, Supporting Table 1). For parallel first order processes, the initial oxidation rate will scale linearly with DOC concentration, while second order kinetics predicts that the initial decay rate scales quadradically 
with DOC concentration. To directly test the kinetic form, we performed serial oxidation experiments under multiple dilutions of filtered Woods Hole Seawater and showed that the initial decay rate scales linearly with concentration [84]. DOC photooxidation in our high-intensity apparatus is better described by parallel first order kinetics (see Supporting Information).

To estimate the isotopic values of the different DOC components, we represent the oxidation progression as a superposition of parallel first order reactions [5]:

$$
g_{i}(t)=\int_{-\infty}^{\infty} \rho_{i}(k) e^{-k t} d k .
$$

The function $g_{i}(t)$ is the amount of ${ }^{i} \mathrm{C}$, where $i=$ carbon isotope 12,13 , or 14 , remaining in the DOC sample after a time $t ; \rho_{i}(k)$ is the amount of ${ }^{i} \mathrm{C}$ associated with a first order rate constant between $k$ and $k+d k$. After correcting for isotopic fractionation, the relevant isotopic ratios are $R(k)=\rho_{i}(k) / \rho_{12}(k)$. The isotopic ratios $R(k)$ along with the mass density $\rho_{12}(k)$ allow us to estimate the distribution of isotopic values. Fractionation is dealt with explicitly as a multiplier of each decay constant (see Supporting Information). To render the problem numerically tractable, we approximate the integral as a set of discrete exponential components and solve for the parameter values that minimize the error between our model and the serial oxidation data. We then compute the best fit solution using 6 exponential components (three each from the inflection points in the $\delta^{13} \mathrm{C}$ and $\delta^{14} \mathrm{C}$ time series), constraining the solution to fall within measured isotopic values of marine organic matter at each depth. To account for sensitivity to errors we preformed our fit with many different realizations of the data. Our estimated isotope distributions (Fig. 4-2) are probability density estimates for the isotopic values of material from an ensemble of 1000 data fits. Further details can be found in the supporting information. We continue to use $\delta^{14} \mathrm{C}$ rather than $\Delta^{14} \mathrm{C}$ because single $\delta^{14} \mathrm{C}$ and $\delta^{13} \mathrm{C}$ values are not connected 

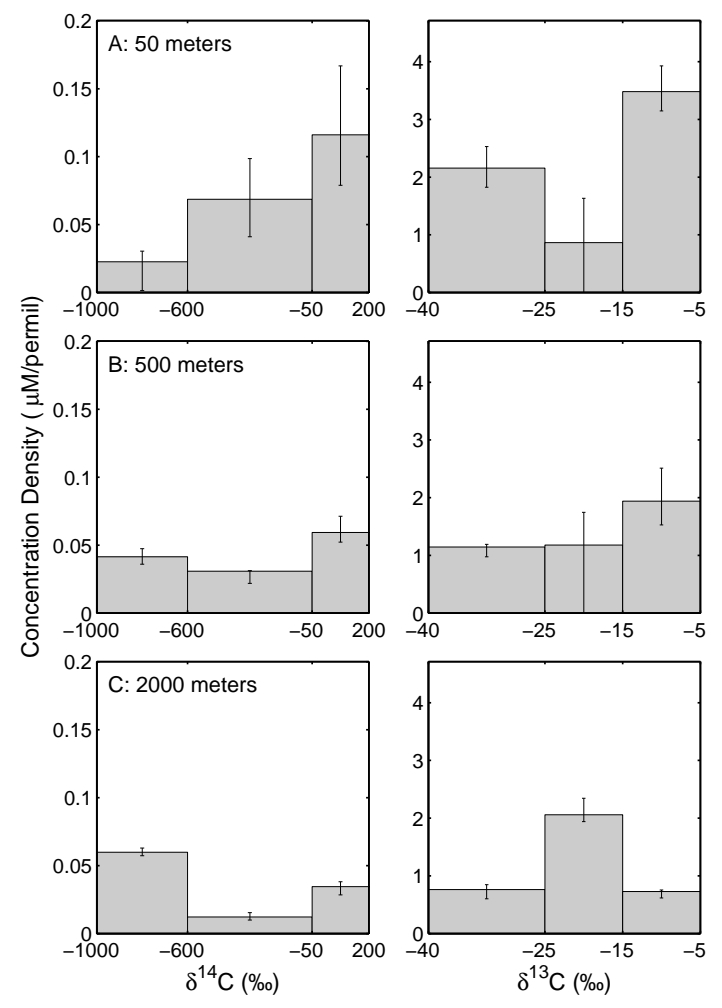

Figure 4-2: Isotopic distributions of DOC: The estimated isotopic distributions for 50 $\mathrm{m}(\mathrm{A}), 500 \mathrm{~m}(\mathrm{~B})$, and $2000 \mathrm{~m}$ (C) depths. The area under each bar corresponds to the average concentration of the enriched $\left(\delta^{14} \mathrm{C} \geq-50 \%\right)$, depleted $\left(-600 \% 0 \leq \delta^{14} \mathrm{C}<\right.$ $-50 \%$ ) , and highly depleted $\left(\delta^{14} \mathrm{C}<-600 \%\right)$, fractions. Error bars are the $25 \%$ and $75 \%$ percentiles, respectively, of our estimate for that fraction. $\delta^{14} \mathrm{C}$ distributions show a preponderance of material at both very enriched and depleted values, with the quantity of enriched material decreasing at depth. $\delta^{13} \mathrm{C}$ distributions show distinct isotopic groups at all three depths. 
between the probability distributions preventing the correction from being calculated. The maximum difference between $\Delta^{14} \mathrm{C}$ and $\delta^{14} \mathrm{C}$ should be less than $\pm 30 \%$ based on the range of $\delta^{13} \mathrm{C}(-40$ to -10$)$ measured in our samples. $\mathrm{DOC} \delta^{14} \mathrm{C}$ for all samples includes modern $(>-50 \%)$ and depleted $(<-50 \%)$ material. The broad range of $(-1000 \%$ to $-50 \%)$ values of depleted DOC suggests a wide range of turnover times for this fraction. In order to frame the interpretation of our results, we further distinguish "less depleted" carbon with $\mathrm{DOC} \delta{ }^{14} \mathrm{C} \geq-600 \%$, the minimum deep Pacific DOC $\Delta^{14} \mathrm{C}$ value, and "highly depleted" DOC with $\mathrm{DOC} \delta^{14} \mathrm{C}<-600 \%$.

Surface seawater contains approximately $30 \mu \mathrm{M}$ radiocarbon modern DOC, which decreases to $15 \mu \mathrm{M}$ at 500 meters and $9 \mu \mathrm{M}$ at 2000 meters. Depleted carbon decreases from $47 \mu \mathrm{M}$ at 50 meters, to $34 \mu \mathrm{M}$ at 500 meters and $31 \mu \mathrm{M}$ at 2000 meters. At first, this decrease in depleted carbon appears at odds with the TCM. However, it is important to note that chemicals with similar decay rates in the ultraviolet oxidation device could have drastically different turnover times in the environment. If this were true, then we would expect the depleted carbon to decrease in concentration, and the the highly depleted portion to increase relative to the less depleted fraction at depth. This is indeed the case, as the less depleted and highly depleted fractions are $9 \mu \mathrm{M}$ and $38 \mu \mathrm{M}$ at 50 meters, $17 \mu \mathrm{M}$ and $17 \mu \mathrm{M}$ at 500 meters, and $7 \mu \mathrm{M}$ and $24 \mu \mathrm{M}$ at 2000 meters. Although we can not rule out an increasing highly depleted fraction, it is most likely due to how isotope values are resolved. The independent $\delta^{13} \mathrm{C}$ distributions show a range of values between $-40 \%$ and $-10 \%$ with a narrowing of the range as a function of increasing depth.

\subsection{Radiocarbon and DOC Cycling}

The presence of enriched, depleted and highly depleted DOC at all depths suggests at least three radiocarbon fractions in DOC with differing potential cycling mechanisms 
(Fig. 4-3). In surface water, the radiocarbon modern fraction has a $\delta^{14} \mathrm{C}$ value consistent with surface water DIC $\Delta^{14} \mathrm{C}$, supporting autochthonous production by marine microbes. At depth, this fraction has a $\delta^{14} \mathrm{C}$ value $>\mathrm{DIC} \Delta^{14} \mathrm{C}$, decoupling its source from either in-situ production by chemoautotrophy or advection from high latitudes, both of which would impart an isotopic value equal to $\mathrm{DIC} \Delta{ }^{14} \mathrm{C}$. The dissolution of sinking particles provides a straightforward mechanism to transfer radiocarbon modern material to the deep ocean [15]. The enriched radiocarbon value suggests that this material has a turnover timescale less than 50 years and is thus semi-labile DOC. This, along with its concentration, $\sim 10 \mu \mathrm{M}$, indicates that the semi-labile fraction dominates the carbon flux through bathypelagic DOC. If deep, semi-labile DOC is in a steady state governed by first order kinetics at least $1 \mathrm{Pg}$ carbon per year flows through this fraction. The carbon flux through this enriched DOC is one to two orders of magnitude higher than the flux through abyssal DOC calculated from bulk radiocarbon and concentration values. Shallow export production is estimated to be $11 \mathrm{Pg} \mathrm{C}$ per year, with between 2.3 and 5.5 Pg C per year falling through 500 $\mathrm{m}[85,86]$. Our measurements suggest that 20 to $50 \%$ of sinking particulate organic matter is solubilized during export and sequestered as semi-labile DOC within the deep ocean.

The depleted DOC fraction would include contributions from in-situ chemoautotrophy, chemolithotrophy $[46,62,87]$ and from the advection of DOC from higher latitudes $[13,16,31]$. It is also likely that some modern and highly depleted carbon is not fully resolved in our measurements, and therefore contributes to the depleted fraction. Highly depleted DOC appears to increase with depth and is near $25 \mu \mathrm{M}$ and $-800 \%$ at $2000 \mathrm{~m}$. This fraction is significantly depleted relative to the mean $\delta^{14} \mathrm{C}$ value for DOC in the deep ocean (-800\% vs. $-522 \%$ respectively), suggesting a radiocarbon age near 12,000 years. Current theories suggest that this refractory portion of DOC survives multiple turnover times and is in an approximate first order 

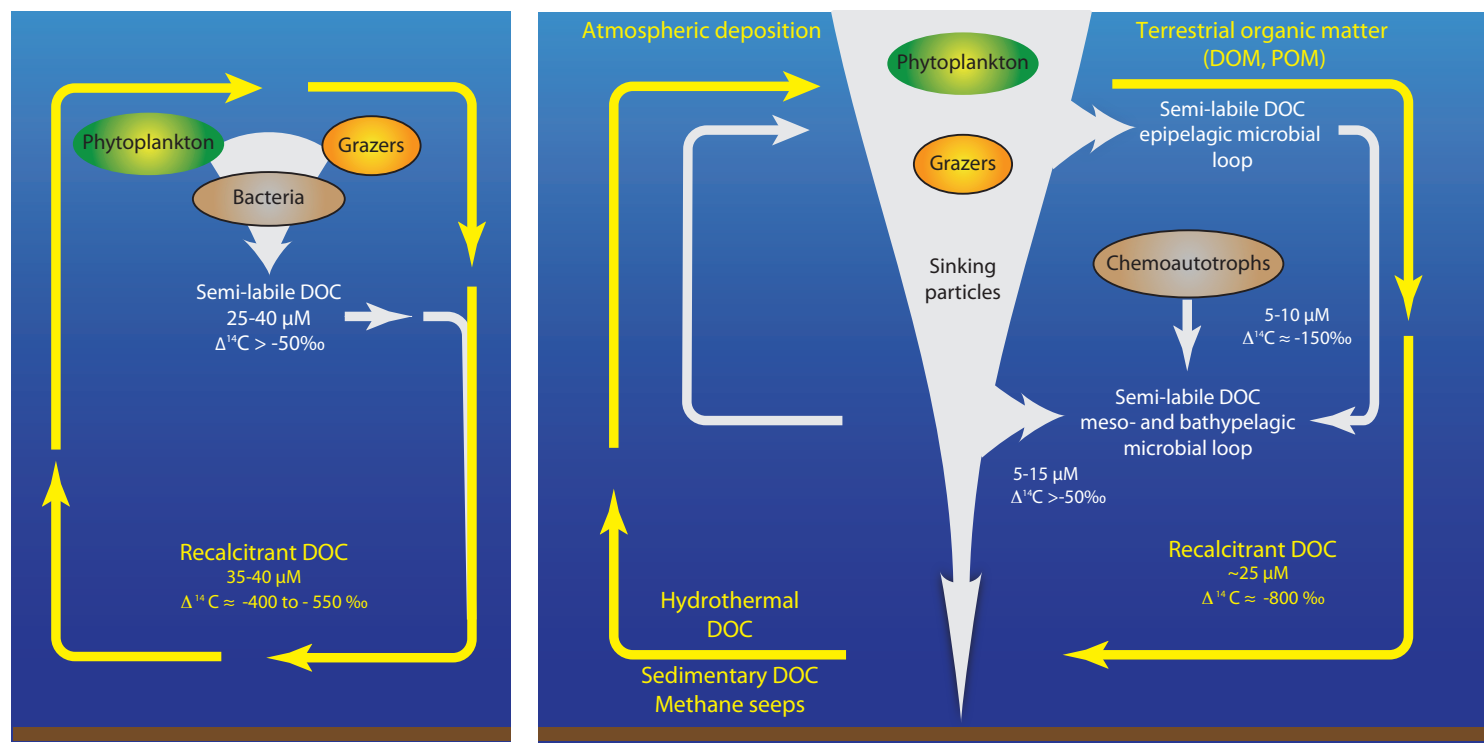

Figure 4-3: Physical interpretation: Schematic contrasting DOC cycling in the twocomponent model (left) and the multi-component model proposed here (right). In the two-component model, DOC with a modern radiocarbon value is added to surface seawater as a by-product of microbial carbon production and cycling, such that surface DOC concentrations are relatively high (typically $60-80 \mu \mathrm{M}$ ) and enriched in radiocarbon $(-200$ to $-400 \%$ ). The semi-labile component of DOC $(25-40 \mu \mathrm{M}$; $\Delta^{14} \mathrm{C}>-50 \%$ ) is removed in the mesopelagic ocean leaving a background fraction of refractory DOC $(35-40 \mu \mathrm{M} ;-400$ to $-550 \%$ ) that cycles through the ocean over several millennia. Our results (right) show that a large fraction $(20-50 \%)$ of semi-labile carbon associated with sinking particles, with a modern radiocarbon value $(>-50 \%$ ), is injected into the deep DOC reservoir (white arrow), where it accumulates and cycles on decadal timescales in a meso-and bathypelagic microbial loop. Chem- and lithoautotrophy, as well as advection also contribute to deep, semilabile DOC. The refractory fraction of DOC (yellow arrow) has a lower concentration and older mean radiocarbon age than predicted by the TCM. Sources of semi-labile and refractory carbon from terrestrial organic matter (atmospheric deposition, rivers, groundwater, desorption from particulate POC), hydrothermal vents, methane, and fossil carbon from seeps, sediments, and the atmosphere are consistent with stable carbon isotopic distributions. 
steady state with modern inputs. In this case, it is important to realize that the radiocarbon age does not equate to the mean age of the DOC. When a reservoir containing a single component with turnover time $\tau$ is in steady state, its mean age equals its turnover time, and the age distribution is exponential [38]. Similar reasoning leads to the following relationship between the radiocarbon age $a_{r}$ of the reservoir and its turnover time $\tau$ (see Supporting Information):

$$
\tau=\frac{e^{\lambda a_{r}}-1}{\lambda}
$$

where $\lambda$ is the decay constant of radiocarbon. Inserting $a_{r}=12,000$ years, we calculate the turnover time $\tau$ to be 30,000 years for refractory DOC. This time scale suggests either DOC cycling takes much longer than the currently believed 6000 years [8], or that an external source of isotopically depleted, (pre-aged), carbon supports the refractory DOC.

\subsection{Global Context}

The current form of the TCM is sufficient to explain many open ocean DOC measurements. Chief among its accomplishments are an explanation for the depleted radiocarbon values found in surface ocean DOC and the slow decrease in DOC concentration between the deep North Atlantic and Pacific Oceans. The fundamental tenant of the TCM, that radiocarbon depleted DOC persists throughout the water column, is well supported. However, certain measurements remain hard to explain unless one allows for a semi-labile reservoir of DOC in the deep ocean.

DOC concentrations for a nominal global ocean transit (Fig. 4-4) show a general decrease consistent with the slow decay models used to explain them [13]. These models use a superposition of material with different first order decay rates to explain the slowdown of net DOC degradation in the ocean. Decay in these model systems is 


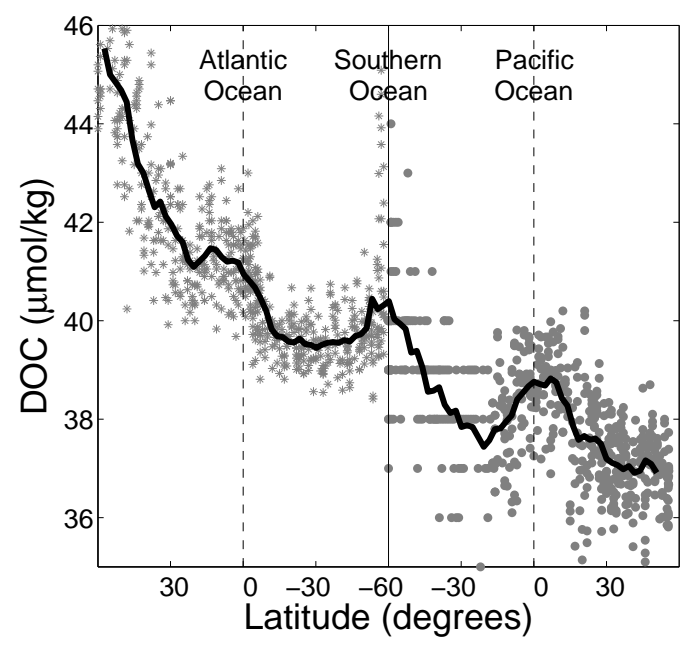

Figure 4-4: Changes in DOC concentration $(\mu \mathrm{mol} / \mathrm{kg})$ : DOC [16] in the core of the North Atlantic Deep Water along Atlantic transect A16 $\left(^{*}\right)$ and in the Circumpolar Deep Water along Pacific transect P16 $(\bullet)$. Water samples were used from the neutral density surfaces consistent with these water masses: $41.25<\sigma_{3000}<41.5$ in the Atlantic and $45.85<\sigma_{4000}$ in the Pacific [16]. The running mean across $15^{\circ} \mathrm{N}-\mathrm{S}$, solid line, is superimposed on the individual DOC measurements, single points. Note the $1-2 \mu \mathrm{M}$ increase in DOC at the equator relative to the background trend in both basins. This is consistent with an increased particle export from enhanced primary production at the equator. 
completely monotonic and fails to accommodate increases in either bulk DOC concentration or decay rate. Deep ocean POC-DOC transfer predicts that in areas of higher export production there should be local elevations in deep sea DOC concentrations due to a larger reservoir of semi-labile DOC. Global DOC data plotted along density surfaces [16] as it travels from the North Atlantic to the North Pacific Ocean (Fig. 4-4) provides evidence for these systematic fluctuations. In equatorial regions and the Southern Ocean we find increases in DOC concentration of $1-2 \mu \mathrm{M}$, or $10-20 \%$ of the semi-labile reservoir. Post nuclear bomb testing radiocarbon values for the deep semi-labile reservoir of DOC suggest a turnover time of $<50$ years for this portion. Any large impulse of semi-labile DOC should thus persist over $15-20^{\circ} \mathrm{NS}$ based on a mean drift in the deep ocean of $0.3-0.4^{\circ}$ per year which is consistent with the observed spatial fluctuations in deep DOC concentrations (Fig. 4-4). The current paradigm assumes that the dynamics of DOC in the deep ocean are advectively controlled; that once photosynthetically derived DOC is exported from the surface ocean it undergoes purely degradative processes. This assumption allows one to calculate the net DOC flux from deep concentration gradients and equate it with the gross carbon flux [13]. If the dissolution of POC supports a semi-labile portion of the deep DOC however, then the gross flux is no longer calculable from deep sea gradients of DOC. Under this scenario the dynamics of deep ocean DOC is affected by surface processes like primary and export production and the flux through this reservoir could be substantially higher. Regional scale changes in the global DOC concentration data support this perspective. A recent reanalysis of global DOC data provides additional evidence that decay in the deep ocean is not monotonic [88]. After accounting for ocean mixing they assert that in the Pacific ocean, the apparent decrease in deep ocean DOC is due entirely to DOC sinks located at mid-water depths in the north Pacific. This breaking of monotonic decay is consistent with our ideas of a steady state for the deep ocean. The apparent sinks are likely due to the decrease in particle 
inputs in the gyre regions relative to the equatorial regions of the Pacific basin [89] and are consistent with the local increase seen in the Pacific portion of figure 4-4.

As modern radiocarbon is continually fed into the deep DOC reservoir through the dissolution of sinking particles, its bulk radiocarbon values should fluctuate accordingly. This effect should be especially clear as one travels from the Sargasso Sea, through the Southern Ocean, and into the North Central Pacific. Along this journey surface ocean DIC ranges from $\Delta^{14} \mathrm{C} \sim 50-150 \%$ in the North Atlantic to $\Delta^{14} \mathrm{C} \sim-100-0 \%$ in the Southern Ocean and $\Delta^{14} \mathrm{C} \sim 0-150 \%$ in the North Pacific [22]. Based on the average concentrations and radiocarbon values in the deep $(>1000 \mathrm{~m})$ ocean at these three sites, a radiocarbon depleted $\left(\Delta^{14} \mathrm{C}=-800 \%\right.$ in the Pacific) background component with a concentration of $25 \mu \mathrm{M}$ would require a semilabile DOC pool with a radiocarbon value of $\left(\Delta^{14} \mathrm{C} \approx 120 \%\right)$ in the Sargasso Sea, $\left(\Delta^{14} \mathrm{C} \approx-80 \%\right)$ in the Southern Ocean, and $\left(\Delta^{14} \mathrm{C} \approx 140 \%\right)$ in the North Central Pacific. These values are consistent with those of surface ocean DIC in these different regions over the last 50 years. If DOC conservatively ages in the deep ocean its radiocarbon age and that of DIC should change at the same rate. Plotting the mean radiocarbon ages of deep water DOC and DIC (Fig. 4-5) in the Sargasso Sea [12], Southern Ocean [90], and North Central Pacific Ocean [12] shows that while data from the North Central Pacific Ocean is consistent with conservative aging, the DOC in the Southern Ocean is $500-1500$ years too old [90]. Figure 4-5 shows that DOC ages more rapidly than DIC as water moves from the North Atlantic to the Southern Ocean, then more slowly than DIC as water moves from the Southern Ocean into the North Pacific. As our simple example demonstrates, this plot is straightforwardly explained by a highly depleted, background DOC component, and a semi-labile fraction which fluctuates in size and radiocarbon value based on fluctuations in surface DIC radiocarbon.

Carbon flux from POC to DOC could provide a unifying framework for DOC 


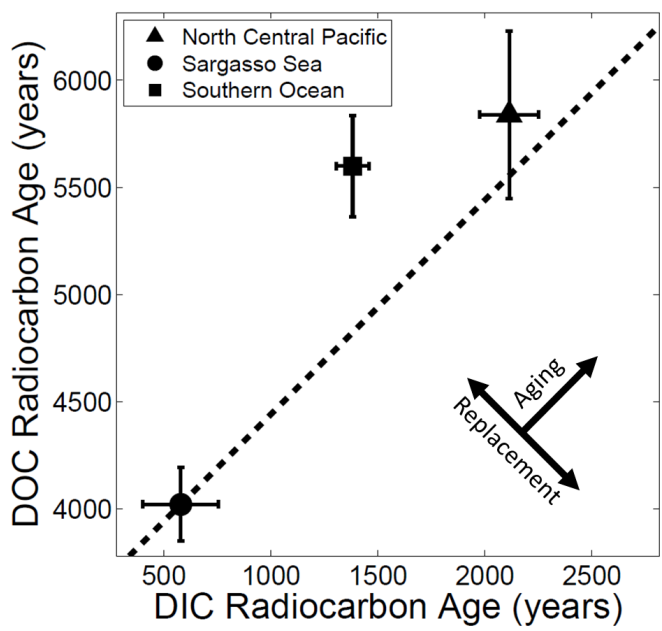

Figure 4-5: Bulk radioisotopes and the TCM: (A) DOC radiocarbon age is plotted versus DIC radiocarbon age for the deep (> 1000 meters) North Central Pacific Ocean [12], Sargasso Sea [12], and Southern Ocean [90]. Mean values are plotted where missing DIC radiocarbon values were linearly interpolated from the depth profile. Error bars represent \pm 1 standard error on the mean. Points on the dotted line are consistent with conservative aging of both DOC and DIC.

cycling in disparate environments. POC-DOC transfer from terrestrial sources are believed to effect the bulk $\delta^{13} \mathrm{C}$ values in places like the Mid-Atlantic bight, Western North Pacific, and Arctic Ocean [46,48,64]. Griffith et al. [46] use isotopic evidence to suggest that $30 \%$ of deep Canada Basin water could be terrestrially derived, which would leave the background, refractory marine-derived fraction at a concentration of $28 \mu \mathrm{M}$, well below DOC values in the deep North Pacific, but similar to the values determined by our measurements. This suggests that deep Pacific DOC $(35-40 \mu \mathrm{M})$ contains $7-12 \mu \mathrm{M}$ semi-labile DOC. The Mediteranean Sea may also be unified within the POC-DOC framework. Although the turnover time for deep water masses is an order of magnitude less in the Mediteranean than in the global oceans (100 vs. 1000 years), deep DOC values reach those found in the North Central Pacific [91]. Unique decay conditions may be present. However, this finding is consistent with the ultra-oligotrophic conditions where these low values are found, and the predicted decrease of deep semi-labile DOC from particles. 


\subsection{Conclusion}

The contrast between the large number of diverse sources that supply carbon to marine DOC, and the isotopic uniformity measured in stable and radiocarbon analyses and inferred from the TCM has long been considered as a paradox in ocean carbon cycling. Our results show that marine DOC is isotopically diverse, with a broad range of potential sources and cycling timescales. Deep DOC $\delta^{13} \mathrm{C}$ ranges between $-40 \%$ o and $-10 \%$, allowing for significant contributions from terrestrial organic matter, black carbon, DOC from hydrothermal sources and methane seeps. Exceptionally efficient sinks for these external sources of carbon do not need to be invoked to explain the isotopic value of DOC in the deep sea. Furthermore, we suggest that the total flux of carbon through DOC in the deep ocean is at least an order of magnitude higher than the net carbon flux derived from abyssal concentration gradients [13] and bulk radiocarbon measurements [8]. Current flux estimates that equate total with net flux assume that inputs from the dissolution of sinking particles and chemoautotrophy are small. Our data suggests otherwise. Active cycling of carbon, and large annual carbon fluxes through DOC are not restricted to the epipelagic ocean but occur throughout the water column (Fig. 4-3). Our work suggests a DOC cycle that is far richer, and potentially far more relevant on human timescales than previously recognized.

\subsection{Supporting Information}

\subsubsection{Materials and Methods}

Seawater was collected using wire-mounted Niskin bottles that had been cleaned and tested for radiocarbon contamination before use. Water was drained directly (without filtration) into clean polycarbonate bottles and frozen. Particulate organic carbon measured at Station ALOHA by the Hawaii Ocean Time-series program immediately 
before and after our sample collection show $2.2-2.3 \mu \mathrm{M} \mathrm{C}$ or $<3 \%$ of TOC at 50 $\mathrm{m}$, and $0.4 \mu \mathrm{M} \mathrm{C}$ or $<1 \%$ of TOC at $350 \mathrm{~m}$, and we expect POC to represent $<1 \%$ of TOC in our $500 \mathrm{~m}$ and $2000 \mathrm{~m}$ samples. Photo-oxidation of these small amounts of POC do not significantly affect our results. Samples were stored at $-20^{\circ} \mathrm{C}$ until analysis.

In the laboratory, the concentration, $\delta^{13} \mathrm{C}, \delta^{14} \mathrm{C}$, and $\Delta^{14} \mathrm{C}$ value were determined at each depth for a given sample. This was done in conjunction with the National Ocean Sciences Accelerator Mass Spectrometry (NOSAMS) facility using a slightly modified version of their standard DOC oxidation protocol. Each bottle was acidified $(\mathrm{pH} \leq 2.5)$ using phosphoric acid, and then split for use in a bulk analysis and in our time series analysis. The water for the bulk analysis was sparged with ultra-high purity helium gas for 1 hour. At this point, it was irradiated with a 1200W, medium pressure mercury arc UV lamp (UV Doctor) for four hours. A water cooling system is employed to keep the pressure of the system below 800 torr. A shutter system was used to allow the lamp intensity to stabilize for 2.5 minutes before starting the irradiation. After this time the carbon dioxide generated from the oxidation was collected on a vacuum extraction line and quantified with a calibrated pressure gauge. We used the maximum sample volume possible $(\sim 1 \mathrm{~L})$ for our measurements. The collected carbon dioxide was then cleaned and analyzed for $\delta^{13} \mathrm{C}$ and $\Delta^{14} \mathrm{C}$ by the NOSAMS staff. Time series measurements were done in a similar fashion. For the first time point, the system was sealed, and the acidified sample was sparged with ultra-high purity helium gas for 1 hour. After the 2.5 minute warmup time the sample was irradiated for the proper time at which point the lamp was turned off and the carbon dioxide collected and quantified. If the internal pressure neared 800 torr, then the cooling system was engaged. This occurred after 8-12 minutes of oxidation. Each time step proceeded in this manner without additional 1 hour sparges. An entire time series took three days to collect, and so the system was kept closed to the atmosphere 
Table 1: Carbon mass and isotope data from the serial oxidation of DOC at Station ALOHA. Full oxidation data is from a duplicate sample that was completely oxidized by four hours of UV irradiation before analysis. The concentration values were blank corrected. Analytic errors from the gas measurement and isotope analysis were less than $\pm 0.2 \mu \mathrm{M}, \pm 0.1 \delta^{13} \mathrm{C}$, and $\pm 11 \delta^{14} \mathrm{C}$. Missing time series data (blanks in table) were interpolated to estimate the average isotope values (indicated with a star $(*))$. These estimates were not used in the time series analysis presented in the main text.

\begin{tabular}{|c|c|c|c|c|c|c|c|c|c|c|c|c|c|}
\hline \multirow{2}{*}{ Data Source } & \multirow[b]{2}{*}{ UV-Time (min) } & \multicolumn{4}{|c|}{50 Meters } & \multicolumn{4}{|c|}{500 Meters } & \multicolumn{4}{|c|}{2000 Meters } \\
\hline & & $\mu \mathrm{M}$ & $\delta^{13} \mathrm{C}$ & $\Delta^{14} \mathrm{C}$ & $\delta^{14} \mathrm{C}$ & $\mu \mathrm{M}$ & $\delta^{13} \mathrm{C}$ & $\Delta^{14} \mathrm{C}$ & $\delta^{14} \mathrm{C}$ & $\mu \mathrm{M}$ & $\delta^{13} \mathrm{C}$ & $\Delta^{14} \mathrm{C}$ & $\delta^{14} \mathrm{C}$ \\
\hline Full Oxidation & 240.0 & 75.8 & -21.3 & -237.9 & -232.2 & 45.2 & -22.1 & -395.7 & -392.0 & 40.0 & -22.7 & -520.0 & -517.7 \\
\hline \multirow{10}{*}{ 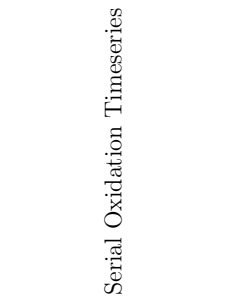 } & 1.5 & 5.1 & -30.3 & -224.5 & -232.9 & 3.9 & -27.5 & -328.4 & -331.8 & 2.9 & -26.6 & -471.4 & -473.1 \\
\hline & 3.6 & 6.5 & -30.9 & -189.9 & -199.7 & 4.4 & -29.5 & -360.9 & -366.8 & 2.8 & -28.7 & -517.9 & -521.5 \\
\hline & 6.3 & 7.1 & -28.5 & -175.4 & -181.4 & 5.1 & -29.1 & -361.4 & -366.7 & 3.3 & -29.1 & -528.6 & -532.5 \\
\hline & 9.9 & 7.9 & -24.3 & -179.3 & -178.1 & 5.7 & -27.0 & -367.7 & -370.4 & 4.0 & -27.9 & -527.1 & -529.9 \\
\hline & 15.4 & 8.2 & $-20.6^{*}$ & $-211.2^{*}$ & $-203.9^{*}$ & 6.5 & -23.0 & -378.4 & -375.8 & 5.0 & $-20.8^{*}$ & $-534.7^{*}$ & $-530.7^{*}$ \\
\hline & 24.1 & 8.5 & -20.8 & -252.8 & -246.2 & 5.9 & $-19.7^{*}$ & $-405.0^{*}$ & $-398.5^{*}$ & 5.7 & -20.8 & -550.4 & -546.5 \\
\hline & 36.6 & 7.6 & -22.4 & -277.8 & -273.9 & 4.5 & -21.0 & -433.7 & -428.9 & 4.4 & -19.8 & -566.9 & -562.2 \\
\hline & 58.3 & 7.5 & -24.3 & -297.5 & -296.5 & 3.8 & -24.4 & -443.5 & -442.7 & 3.3 & -24.2 & -570.5 & -569.7 \\
\hline & 91.9 & 6.1 & -21.8 & -319.8 & -315.3 & 3.0 & -25.6 & -431.0 & -431.7 & 2.4 & -26.7 & -549.6 & -551.1 \\
\hline & 240.0 & 8.0 & 2.1 & -310.6 & -271.8 & 3.8 & -4.2 & -448.0 & -424.1 & 3.5 & -10.4 & -533.4 & -519.2 \\
\hline \multirow[t]{2}{*}{ Bulk from Timeseries } & 240.0 & 72.4 & $-21.6^{*}$ & $-244.2^{*}$ & $239.3^{*}$ & 46.5 & $-23.2^{*}$ & $-392.4^{*}$ & $-390.6^{*}$ & 37.4 & $-23.4^{*}$ & $-537.2^{*}$ & $-535.3^{*}$ \\
\hline & & \multicolumn{4}{|c|}{50 Meters } & \multicolumn{4}{|c|}{637 Meters } & \multicolumn{4}{|c|}{1808 Meters } \\
\hline \multicolumn{2}{|c|}{ North Pacific Subtropical Gyre $[8,12]$} & 75 & -20.9 & -145 & -138 & 42 & $-20.8^{*}$ & -412 & $-407^{*}$ & 34 & -20.7 & -511 & -507 \\
\hline
\end{tabular}

with an over-pressure of around 40 torr during the night hours. Data is shown in 1.

Serial uv-oxidation experiments on oceanic DOC to date have been reported by a single additional group. Beaupre et al. [23,43] take their samples from Station M (Eastern Pacific) for both the surface and deep waters. Unfortunately, $\delta^{13} \mathrm{C}$ was not reported making a direct comparison impossible. The main difference is the mass distribution across the time-series. Beaupre et al. oxidize half of the material in the first time point which leads to the apparent lack of fluctuations in the radiocarbon time series. Despite this, a simple two component exponential model of their deep water data [23] fits quite well $\left(\mathrm{R}^{2}=.997\right)$, suggesting that approximately $40 \%$ of the carbon in the deep ocean has a radiocarbon value below $-720 \%$ and that two exponentials are sufficient to explain their mass timeseries. This is in principle the power of using $\delta^{14} \mathrm{C}$ and $\delta^{13} \mathrm{C}$. Mass only has logarithmic resolution in rate constant space. It is the fluctuations in $\delta^{14} \mathrm{C}$ and $\delta^{13} \mathrm{C}$ which allow us to access these intermediate rate constants. It should be noted here that although $\Delta^{14} \mathrm{C}$ is an appropriate correction for a single fractionation event, in general isotopic fractionation is non-linear and the 
Table 2: Carbon mass and isotope data from a set of replicate samples from $500 \mathrm{~m}$. This is a table of two seawater samples from the same bottle run in sequence. The table contains the oxidation time, the values at that time for each replicate, and the statistical error between the two replicates. The statistical error is the deviation between the two time series corrected for the mean deviation. The bulk values are the total time, concentration, and mean isotope values or deviations. The mean statistical errors of $0.3 \mu \mathrm{M}, 1.6 \delta^{13} \mathrm{C}$, and $12.7 \delta^{14} \mathrm{C}$ are consistent with our perturbation ranges of $0.4 \mu \mathrm{M}, 1.2 \delta^{13} \mathrm{C}$, and $20 \delta^{14} \mathrm{C}$ for our Monte-Carlo analysis. Note that the error value for $\delta^{13} \mathrm{C}$ is dominated by the final deviation.

\begin{tabular}{|c|c|c|c|c|c|c|c|c|c|c|c|c|c|}
\hline \multirow[b]{2}{*}{ Data Source } & \multirow[b]{2}{*}{ UV-Time (min) } & \multicolumn{4}{|c|}{ Replicate 1} & \multicolumn{4}{|c|}{ Replicate 2} & \multicolumn{4}{|c|}{ Statistical Error } \\
\hline & & $\mu \mathrm{M}$ & $\delta^{13} \mathrm{C}$ & $\Delta^{14} \mathrm{C}$ & $\delta^{14} \mathrm{C}$ & $\mu \mathrm{M}$ & $\delta^{13} \mathrm{C}$ & $\Delta^{14} \mathrm{C}$ & $\delta^{14} \mathrm{C}$ & $\mu \mathrm{M}$ & $\delta^{13} \mathrm{C}$ & $\Delta^{14} \mathrm{C}$ & $\delta^{14} \mathrm{C}$ \\
\hline$\cdot \stackrel{\mathscr{E}}{\vec{E}}$ & 3.6 & 3.2 & -29.4 & -362.0 & -363.6 & 2.6 & & -361.0 & -356.2 & 0.7 & & 16.4 & 12.2 \\
\hline$\stackrel{d}{0}$ & 6.3 & 2.6 & -29.9 & -381.0 & -382.8 & 2.6 & & -365.0 & -360.1 & 0.1 & & 1.4 & -3.1 \\
\hline$\exists$ & 9.9 & 3.5 & -29.5 & -371.0 & -372.7 & 3.3 & & -366.0 & -361.3 & 0.3 & & 12.4 & 8.2 \\
\hline$\approx$ & 15.4 & 5.0 & -27.9 & -365.0 & -363.9 & 4.8 & -28.7 & -361.0 & -360.7 & 0.3 & -0.5 & 13.4 & 16.5 \\
\hline$\stackrel{0}{=}$ & 24.1 & 6.3 & -24.0 & -386.0 & -380.7 & 6.4 & -25.2 & -360.0 & -355.9 & 0.0 & -0.1 & -8.6 & -5.2 \\
\hline$\frac{\pi}{\pi}$ & 36.6 & 5.3 & -20.3 & -415.0 & -405.5 & 5.8 & -20.5 & -378.0 & -367.3 & -0.4 & -1.1 & -19.6 & -18.5 \\
\hline ○் & 58.3 & 4.1 & -21.1 & -436.5 & -427.8 & 5.2 & -20.1 & -405.0 & -394.9 & -1.0 & -2.3 & -14.1 & -13.3 \\
\hline$\widetilde{\Xi}$ & 91.9 & 3.8 & & & & 3.5 & -23.7 & -418.0 & -411.9 & 0.4 & & & \\
\hline 樆 & 240.0 & 5.6 & -14.1 & -461.0 & -445.1 & 5.6 & -19.4 & -419.0 & -408.3 & 0.1 & 4.0 & -24.6 & -17.2 \\
\hline Bulk from Timeseries & 240.0 & 41.8 & -23.9 & -396.7 & -391.5 & 42.3 & -24.5 & -378.4 & -371.8 & 0.3 & 1.6 & 14.9 & 12.7 \\
\hline
\end{tabular}

underlying distribution of potential rate constants and fractionation factors is best directly taken into account as done here.

It is important to our conclusions that the fluctuations we find in the isotopic time series are real and not artifacts of our analysis. Two types of errors could effect our time series; systematic (from blanks) and statistical errors. Large systematic errors appear insignificant to our $\delta^{14} \mathrm{C}$ time series as the deviation between the bulk oxidation and mean value from the time series is less than $20 \%$ for all three depths. This is consistent with the standard deviation (23\%) for bulk samples taken from the same site and analyzed on the NOSAMS apparatus [46]. Systematic errors due to a correlation between sample time and mass [23] were mitigated by choosing time points in order to collect approximately equal portions of the sample at each step. If the data trends seen here were due to systematic errors we would expect to see the largest fluctuations in the deep water sample where concentrations are lower. The largest fluctuations are found in the surface water sample, contrary to this hypothesis. The strongest evidence we have for the reality of our isotope fluctuations is the 
correspondence in the structure of our time series between the surface, middle and deep waters. To quantify this we use the differences between ensuing data points in table 1 (including the estimated values) to get an estimate of the local derivatives. We focus on the 500 and 2000 meter samples as their total concentration differs by less than $20 \%$ and they have a correlation coefficient of .82. A plot of both sets of derivatives corroborates that there are two inflection points and one local minima in both time series. We find that the derivatives differ between the two depths by an average value of $5 \%$ per time point and a have standard deviation of $11 \%$ with a maximum deviation from the mean of $20 \%$. This is consistent with our perturbation errors of $\pm 10 \%$ in our Monte-Carlo analysis. Any larger statistical errors are inconsistent with the strong correspondence between these two time series.

The reproducibility of our time series was tested using two portions from the same 500 meter station (2). The samples were analyzed two years after the first suite of time series analysis, and the data may be influenced by changes in the uv-lamp power and output. Systematic errors between the two time series were $0.5 \mu \mathrm{M}, .6 \% 0 \delta^{13} \mathrm{C}$, and $20 \%$ o $\delta^{14} \mathrm{C}$. These systematic shifts are small relative to the ranges in radiocarbon and stable carbon found in our analysis. The mean statistical errors were $0.3 \mu \mathrm{M}, 1.6 \%$ $\delta^{13} \mathrm{C}$, and $12.7 \% \delta^{14} \mathrm{C}$. These errors are consistent with the perturbation ranges used in our Monte-Carlo analysis of $0.4 \mu \mathrm{M}, 1.2 \% \delta^{13} \mathrm{C}$, and $20 \% 0 \delta^{14} \mathrm{C}$. We can further check consistency between the radiocarbon distributions between time-series from the same depth and location. Performing our data inversion on the first replicate, and comparing it with the inversion from the 500 meter sample used in the main text, (Fig. 4-6), demonstrates the robustness of the two widely distributed groups of radiocarbon. The second replicate was not used because of the missing stable isotope data. The larger errors in the distribution are most likely due to the missing time point 9 in the replicate time series and the poor resolution it created in the derivative there. 


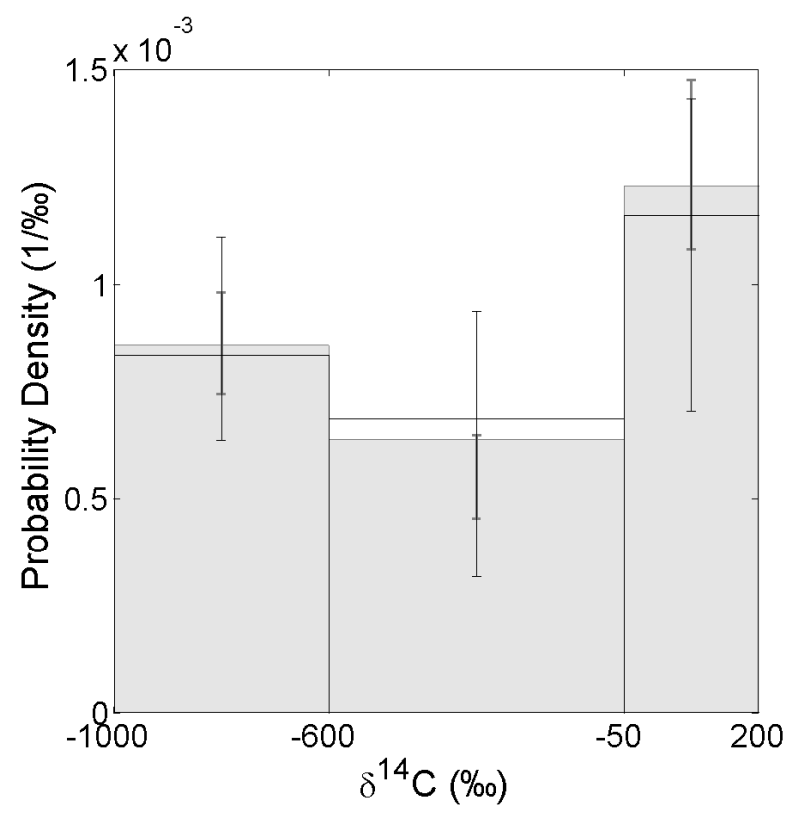

Figure 4-6: Reproducibility: The radiocarbon probability distributions from the 500 meter sample in the main manuscript (grey) and the replicate (white) taken two years later. Both distributions are within errors ( $25 \%$ and $75 \%$ confidence interval) of each other.

\subsubsection{DOC Photo-oxidation Kinetics}

The oxidation of DOC by ultraviolet radiation has long been considered a parallel first order process $[23,24]$ where the concentration,

$$
g=\int_{0}^{\infty} \alpha \rho(k) e^{-k t} d k,
$$

is a function of the rate distribution, $\rho(k)$, and a scale factor, $\alpha=g(t=0)$. The time derivative

$$
\dot{g}=-\alpha \int_{0}^{\infty} k \rho(k) e^{-k t} d k
$$

is proportional to the initial concentration. For the parallel second order case

$$
g=\int_{0}^{\infty} \frac{\alpha \rho(k)}{1+\alpha \rho(k) k t}
$$


where

$$
\dot{g}=\frac{-\alpha^{2} \rho^{2}(k)}{(1+\alpha \rho(k) k t)^{2}} .
$$

Initially, this derivative is proportional to the square of the concentration,

$$
\left.\dot{g}\right|_{t=0}=-\alpha^{2} \int_{0}^{\infty} k \rho^{2}(k) d k .
$$

We can distinguish between the two cases by plotting the initial rate, $\left.\dot{g}\right|_{t=0}$, versus the scale factor, $\alpha$, or initial concentration. For a given sample of DOC at differing dilutions, varying $\alpha$ 's, the initial rate should be well fit by a line through the origin,

$$
\left.\dot{g}\right|_{t=0}=c_{1} \alpha
$$

if the reaction is parallel first order ( $c_{1}$ is a constant), and by a parabola with its vertex at the origin,

$$
\left.\dot{g}\right|_{t=0}=c_{2} \alpha^{2}
$$

if the reaction is parallel second order ( $c_{2}$ is a constant).

We uv-oxidized seawater with a given DOC concentration, and then repeated the experiment at different DOC dilutions. We plot the best estimate of the initial decay rate, based on the amount of DOC lost after 10 minutes of oxidation, versus the initial concentration (Fig. 4-7). The data is much better fit by a line through the origin than by a parabola suggesting that DOC degradation by ultra-violet light is a parallel first order reaction rather than a second order reaction as previously reported [43]. Beaupré et al. [43] use the additional evidence that the decay of a pure organic compound, sucrose, is consistent with second order kinetics while only consisting of a single molecule. This can be explained by understanding the reaction conditions used in DOC oxidation experiments. Sucrose is unstable in water, and under acidic conditions hydrolyzes to fructose and glucose with a half life between 


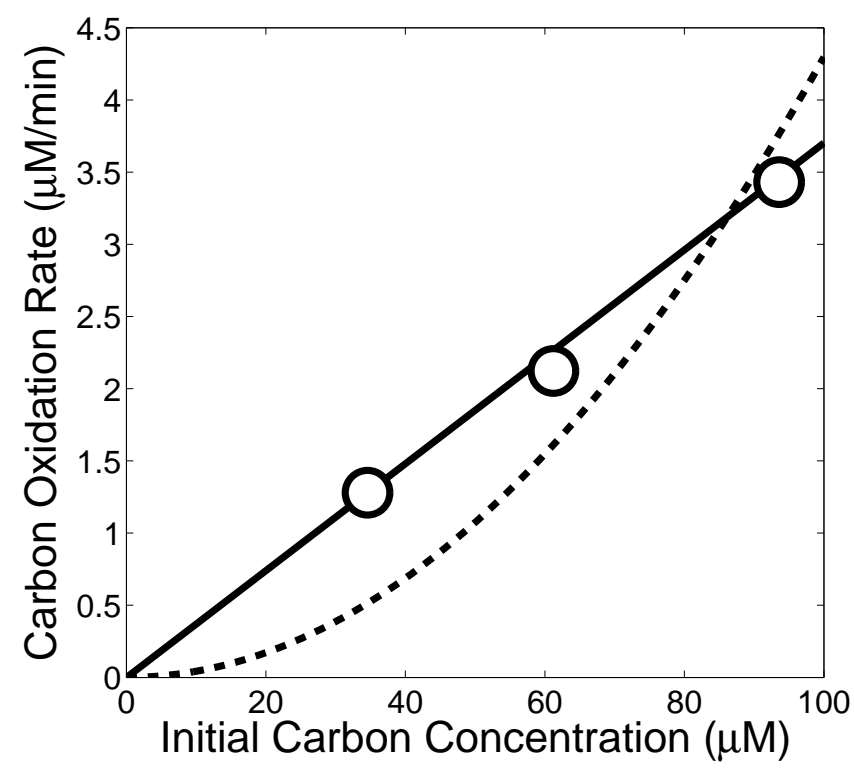

Figure 4-7: DOC photo-oxidation kinetics: Filtered surface water from Woods Hole was uv-oxidized under three different dilutions with fully oxidized seawater leading to concentrations of 94,61 , and $35 \mu \mathrm{M}$. The initial decay rate from a ten minute oxidation, $\circ$, is plotted against the initial concentration for three different dilution experiments. A parallel first order reaction predicts a line going through the origin (solid line, least-squares, $R^{2}=1$ ) whereas a parallel second order process predicts a parabola with a vertex at the origin (dashed line, least-squares, $R^{2}=.85$ ). Ultraviolet oxidation of DOC under ultraviolet light is not consistent with a parallel second order reaction. Errors are contained within the symbols. Although the concentration for each experiment was different, the chemical composition remained constant.

1-10 hours depending on temperature [84]. It is expected that uv-oxidation of sucrose will follow parallel first order kinetics as at least three species (sucrose, fructose, and glucose) would exist in solution.

\subsubsection{Estimating the Isotopic Distribution}

Estimating the isotopic distribution from our time series data requires a kinetic model, (how compounds decay under ultraviolet light), and a method for assigning an isotope value to the mass at a given rate constant. Inverting for a continuous distribution of rates, a method which has been successfully applied to environmental degradation 
problems [5], is highly sensitive to noise in the time series data and is accomplished using methods of regularization. With the addition of isotopic data to the decay time series, these methods are not directly applicable and thus the inherent sensitivity to noise was directly evaluated using a Monte-Carlo approach. We first simplify the continuous space, which has in principle an unlimited number of compound groups, into six discrete components. Six was chosen as the smallest number of components required to match the number of inflection points in our time series data, but not as a suggestion that only six compounds make up DOC. The isotope values, masses, rate constants, and system fractionation factors were then fit starting from a random initial condition. The best fit to the data was found using gradient search methods; multiple initial conditions were employed to find the global best fit. This fitting procedure was performed for many realizations of the data, produced by random perturbations of the data within error bounds, and the probability distribution for mass as a function of isotope value was calculated from the many data fits. A mathematical presentation of this process follows.

As stated in the main text, the kinetic model for the ultraviolet oxidation experiment is

$$
g_{i}(t)=\int_{-\infty}^{\infty} \rho_{i}(k) e^{-k t} d k
$$

The function $g_{i}(t)$ is the amount of ${ }^{i} \mathrm{C}$, where $i=12,13$, or 14 , remaining in the DOC sample after a time $t ; \rho_{i}(k)$ is the amount of ${ }^{i} \mathrm{C}$ associated with a first order rate constant between $k$ and $k+d k$. We seek $\rho_{i}(k)$ given $g_{i}(t)$. We use an $n$-component model to approximate $\rho_{i}(k)$ :

$$
\rho_{i}(k)=\sum_{j=1}^{n} r_{i j} A_{j} D\left(k-\nu_{i j}\right)
$$

where $A_{j}$ is the amount of material in the $j$ th component, $r_{i j}$ is its isotopic ratio, $\nu_{i j}$ is its decay constant, and $D$ is the Dirac delta function . Combining equations (4.10) 
and (4.11) yields

$$
g_{i}(t)=\int_{-\infty}^{\infty}\left(\sum_{j=1}^{n} r_{i j} A_{j} D\left(k-\nu_{i j}\right)\right) e^{-k t} d k
$$

which simplifies into the sum

$$
g_{i}(t)=\sum_{j=1}^{n} r_{i j} A_{j} e^{-\nu_{i j} t} .
$$

The rate constants $\nu_{i j}$ can be further simplified by using an isotopic fractionation factor $\alpha_{j} \simeq 1$ where

$$
\nu_{12, j}=\frac{\nu_{13, j}}{\alpha_{j}}=\frac{\nu_{14, j}}{\alpha_{j}^{2}}
$$

Data from each experiment was collected at a set of $m$ time points, $t_{l}$, where $l=1, \ldots, m$. We denote the mass of ${ }^{i} \mathrm{C}$ collected at time $t_{l}$ by $G_{i}\left(t_{l}\right)$. Isotopes other than ${ }^{12} \mathrm{C}$ make up less than $1 \%$ of carbon atoms and so $G_{12}\left(t_{l}\right)$ approximates the total mass to excellent approximation. Thus, in the experiment, we measure the mass collected, $G\left(t_{l}\right)=G_{12}\left(t_{l}\right)$, and the isotopic ratio of that material, $R_{i}\left(t_{l}\right)$, defined as

$$
R_{i}\left(t_{l}\right)=\frac{G_{i}\left(t_{l}\right)}{G_{12}\left(t_{l}\right)}
$$

We fit the data $G\left(t_{l}\right)$ and $R_{i}\left(t_{l}\right)$ with our model functions $\hat{R}_{i}\left(t_{l}, A_{j}, \nu_{i j}, r_{i j}, \alpha_{j}\right)$ and $\hat{G}\left(t_{l}, A_{j}, \nu_{i j}\right)$. These are defined as

$$
\hat{G}\left(t_{l}, A_{j}, \nu_{i j}\right)=g_{12}\left(t_{l-1}\right)-g_{12}\left(t_{l}\right)
$$

and

$$
\hat{R}_{i}\left(t_{l}, A_{j}, \nu_{i j}, r_{i j}, \alpha_{j}\right)=\frac{g_{i}\left(t_{l-1}\right)-g_{i}\left(t_{l}\right)}{g_{12}\left(t_{l-1}\right)-g_{12}\left(t_{l}\right)}
$$

to account for the discrete nature of the data sampling.

We seek the set of $r_{i j}$ and $A_{j}$ which, when used to compute $\hat{G}\left(t_{l}, A_{j}, \nu_{i j}\right)$ and 
$\hat{R}_{i}\left(t_{l}, A_{j}, \nu_{i j}, r_{i j}, \alpha_{j}\right)$, best fits the data $G\left(t_{l}\right)$ and $R_{i}\left(t_{l}\right)$. This fit minimizes the function

$F=\sum_{l=1}^{n} \frac{1}{N_{12}}\left(\frac{\hat{G}\left(t_{l}\right)-G\left(t_{l}\right)}{e_{12}\left(t_{l}\right)}\right)^{2}+\frac{1}{N_{13}}\left(\frac{\hat{R}_{13}\left(t_{l}\right)-R_{13}\left(t_{l}\right)}{e_{13}\left(t_{l}\right)}\right)^{2}+\frac{1}{N_{14}}\left(\frac{\hat{R}_{14}\left(t_{l}\right)-R_{14}\left(t_{l}\right)}{e_{14}\left(t_{l}\right)}\right)^{2}$

where $N_{i}$ is the number of data points for isotope $i$ and $e_{i}$ is the error estimate for those measurements. For each exponential component, $j$, we have 5 parameters to fit: $A_{j}, r_{2 j}, r_{3 j}, \alpha_{j}$, and $\nu_{12, j}$. We constrain $A_{j} \geq 0,0.96 \leq r_{2 j} \leq 0.99,0 \leq r_{3 j} \leq 1.15$, and

$0.95 \leq \alpha_{j} \leq 1$. The fractionation factor was constrained based on prior laboratory experiments on organic compounds under ultraviolet light [24]. We constrain the mean isotope values from the model to match the results from the bulk measurement. We have access to 31 data points in our experiment including the bulk measurements and require 4-7 exponential components to match the time series, based on the number of turning points in $R_{i}\left(t_{l}\right)$. We used $n=6$ exponential components. Because the solution is sensitive to any noise in the data (the problem is ill-posed), we compile an ensemble of solutions and find their distribution in isotope space.

The computation of this distribution accounts for measurement errors as follows. We preform an analysis for 1000 random perturbations of the data, $G\left(t_{l}\right)$ and $R_{i}\left(t_{l}\right)$ within the bounds of $\pm e_{i}\left(t_{l}\right)$. For each data set we minimized $F$, starting from 10 different random initial guesses of the solution. From this set of 10, the best parameter set was kept. At the end of our calculations we had a set of 1000 solutions. The isotope distributions were estimated as histograms of these solutions.

\subsubsection{Radiocarbon Turnover Time}

A population of DOC molecules has an age structure with both a set of discrete ages, $a$, and a mean age $\bar{a}$. The ages of the different chemical groups are reflected in the radiocarbon age, $a_{r}$, which is calculated from the measured isotopic ratio for 
the sample, $R$. The radiocarbon ratio, $r(a)$, of a sample of carbon with no material exchange changes as a function of its age, $a$, through radioactive decay as

$$
r(a)=e^{-\lambda a}
$$

where $\lambda$ is the decay constant for ${ }^{14} \mathrm{C}$ and $r(0)=1$. The radiocarbon age, $a_{r}$, is defined in terms of the measured isotopic ratio $R$ as

$$
a_{r} \equiv \frac{-\ln R}{\lambda}
$$

When a reservoir of carbon contains a single, non-cycling component the radiocarbon age and the mean age are equivalent: $\bar{a}=a_{r}$. Once the carbon reservoir is cycling, equation (4.19) does not apply because a distribution of ages exist inside any given sample. In this case, the measured isotopic ratio, $R$, is an average isotopic ratio wherein $r(a)$ is weighted by the probability $P(a)$ that any given molecule has a certain age. Therefore

$$
R=\int_{0}^{\infty} P(a) r(a) d a
$$

If a reservoir contains a single component in first-order steady state, then the age distribution is [38]

$$
P(a)=\frac{1}{\tau} e^{-a / \tau}
$$

where $\tau$, the turnover time, equals $\bar{a}$. Substituting equations (4.19) and (4.22) into equation (4.21) then yields

$$
R=\int_{0}^{\infty} \frac{1}{\tau} e^{-a / \tau} e^{-\lambda a} d a
$$


Solving for the turnover time $\tau$, we obtain

$$
\tau=\frac{1-R}{R \lambda} .
$$

Using equation (4.20), we find $\tau$ in terms of the radiocarbon age:

$$
\tau=\frac{e^{\lambda a_{r}}-1}{\lambda} .
$$

Substituting $a_{r}=12,000$ years, and $\lambda=1 / 8267 \mathrm{yr}^{-1}$ we find $\tau=30,000$ years. 


\section{Chapter 5}

\section{Heterogeneous Rate Distributions}

\section{and their Effect on the Cycling of \\ Organic Carbon in Sediments}

Over half of Earth's primary production occurs at sea, with an array of diverse processes controlling the rates at which carbon is remineralized. Despite this diversity, the rate constant of organic matter remineralization scales with the inverse of time, in a power law spanning from minutes to millions of years. Processes regulating this decay in ocean sediments are considered to be based on either the physical association of carbon with minerals, or chemical heterogeneity. This work focuses on a specific form of physical protection which requires the decay process to be diffusion limited, with the limiting step being access to extra-cellular enzymes. We extend this hypothesis to predict the linearity between organic material and sediment surface area. The remainder of the chapter is spent on experimental attempts to directly measure the diffusion coefficient of enzymes in sediment and test diffusion limitation.

\section{$5.1 \quad$ Introduction}

Earth's carbon is repeatedly cycling through the photosynthetic production of organic matter and its subsequent decay by processes known collectively as respiration or remineralization. Approximately half of this process occurs in the ocean. Once fixed, 
the rates at which organic carbon is respired determine atmospheric carbon dioxide levels at short time scales and oxygen levels at long, geologic time scales [92-94].

A multitude of biological, chemical, and physical processes operating over a vast range of time scales control these rates. The fastest processes occur immediately after production of organic matter and before deposition in sediment [1]. Once deposited, organic matter continues to be consumed, first by aerobic respiration and later by a series of anaerobic processes [1]. These processes continue for millions of years [93,94], and any organic matter that survives degradation is then effectively preserved and sequestered in sedimentary rocks [94].

Work on organic matter preservation often focusses on the biological and chemical aspects of the problem [11] leaving behind tantalizing suggestions that the physical substrate must also be involved $[95,96]$. It is probable that physical heterogeneity influences the rates of degradation and preservation once organic matter is deposited in sediments. Recent theoretical work suggests that the spatial distribution of organic matter within organo-clay aggregates may regulate long-term degradation and preservation rates $[40]$.

Particulate organic carbon spends its life in close proximity, and associated with organic material. In the ocean, this association is found beginning in the surface ocean. Organic material is produced in conjunction with biogenic minerals like calcite and silicate $[97,98]$. Additionally, particulate organic carbon incorporates lithogenic minerals from both dust and riverine inputs [99]. This association between minerals and organic carbon has been posed as a mechanism to drive organic matter flux into the deep ocean [100]. The organic material is either protected from degradation through its association with minerals, or the mineral substrate acts as ballast material to drive an increase in sinking rates.

In sedimentary systems, the theory of protection by mineral association has strong support from the linear relationships found between the mineral surface area in a 
sediment, and its organic carbon content $[95,96]$. These relationships are even found in the rock record in shales [101]. In the case of surface area correlations with organic matter, the question of correlation as compared to causation is extremely important.

If mineral surface area is a master variable for organic carbon, then lithogenic inputs to the ocean could be extremely important for carbon sequestration in sedimentary systems. For example, it is estimated that nearly $.5 \mathrm{Pg}$ of dust enter the ocean each year [102]. If organic material associated with these grains at the often observed mololayer equivalent [95] then nearly ten percent of the yearly carbon burial of .14 Pg of carbon [103] could be due to dust fluxes alone. However, if the correlations are not causal, then processes like dust deposition could have no direct effect on the decay and preservation of organic material in sediments. If the association of organic material with minerals was a master variable for organic material decay, one could imagine building a paradigm for organic matter cycling where the long term fate of organic material was modulated by its access to minerals.

Theories which attempt to include mineral surfaces in their formulation have multiple types of observations with which to test the consistency of the approach. Two observations seem especially pertinent. Any theory should be able to explain the power-law relationship between the apparent first order rate constant of decay, $k$, and the age of the organic material

$$
k \propto \frac{1}{t}
$$

This relationship appears to hold for over ten orders of magnitude in time [6]. Additionally, it would make sense that the model extend toward an understanding of the linear relationship found between organic matter content and sediment mineral surface area.

We explain the findings and assumptions of the Rothman-Forney model for organic 
matter decay below [40]. We then extend the theory to provide a mechanism for explaining the linear relationship between organic matter content and surface area. Additionally, we explore the possible connection between the slowdown in degradation rates with depth and the decrease in organismal density. Most explanations for the protective capability of minerals focus on either steric hindrance, or the existence of microscale pores which hide organic material. A conceptual expansion of the Rothman-Forney model suggests that surface area acts a lot like an impedance. When organic material and surface area find themselves occupying the same environment, the surface area interacts with organisms and their extracellular enzymes to impede degradation. In this way, organic carbon could be viewed as on a degradation-mineral association axis where the state of mineral association provides a governing control on the decay distribution.

\subsection{Theory: A Physical Model of Organic Matter Degradation}

Organic matter degradation results from a complex series of biological, chemical, and physical processes. Rates depend on metabolism (aerobic or anaerobic), mechanisms of enzymatic hydrolysis, and the composition of microbial and macrofaunal communities. Relevant chemical properties range from the oxidation state of the environment to the intrinsic "reactivity" of various organic compounds. Physical depositional processes link biological and chemical parameters to the depositional environment, as in the association of clay and organic matter [93, 95, 99].

This inherent complexity confounds attempts to determine specific rates of decay. Indeed, a salient feature of organic carbon decay is the apparent lack of a single rate at which decay occurs. This phenomenon was strikingly shown over two decades

ago by Middelburg [104], who demonstrated that apparent first order decay constants 
systematically decrease as organic matter ages. As a consequence, the decay of organic matter is slower than exponential. Why such sub-exponential decay occurs, however, remains in question.

Proposed regulatory mechanisms for organic matter decay fall into two main categories: intrinsic chemical reactivity $[11,105]$ and the association of organic matter with minerals [106-109]. The intrinsic reactivity hypothesis is straightforward. Initially, decay is fast as easily degraded, labile compounds dominate the rate. Once these compounds are eliminated, the remaining, more recalcitrant compounds decay at slower and slower rates leaving behind a pool of nonreactive compounds [110]. The mineral protection hypothesis are based on the strong correlations in shelf sediments between mineral surface area, mineralogy, and organic carbon $[93,96]$, further substantiated by the apparent lack of change in chemical composition of organic matter and intrinsic reactivity with time $[106,111]$. The physical protection theory, however, makes no prediction for the functional form of sub-exponential decay.

Recent theoretical work in Rothman's group suggests that physical mechanisms alone could suffice to produce sub-exponential decay [40]. The Rothman-Forney (RF) model consists of a few key parts: microbes which release extra-cellular enzymes and minerals which impede the free diffusion of enzymes to organic matter (fig. 51). The only heterogeneities present in the model are physical. Differences in the reactivity of organic matter depend on its accessibility to hydrolytic enzymes, not on any intrinsic chemical properties. In this way stationary gradients in the concentration of extracellular enzymes provide a wide distribution in reaction rates consistent with subexponential decay.

The model supposes that microbes are randomly distributed in a porous medium of porosity $\phi$ where the characteristic pore size is small compared to the spacing between microbes. TEM images taken in sediment by Bennett corroborate this assertion [96]. Organic matter is associated with mineral surfaces and thus held stationary in the 


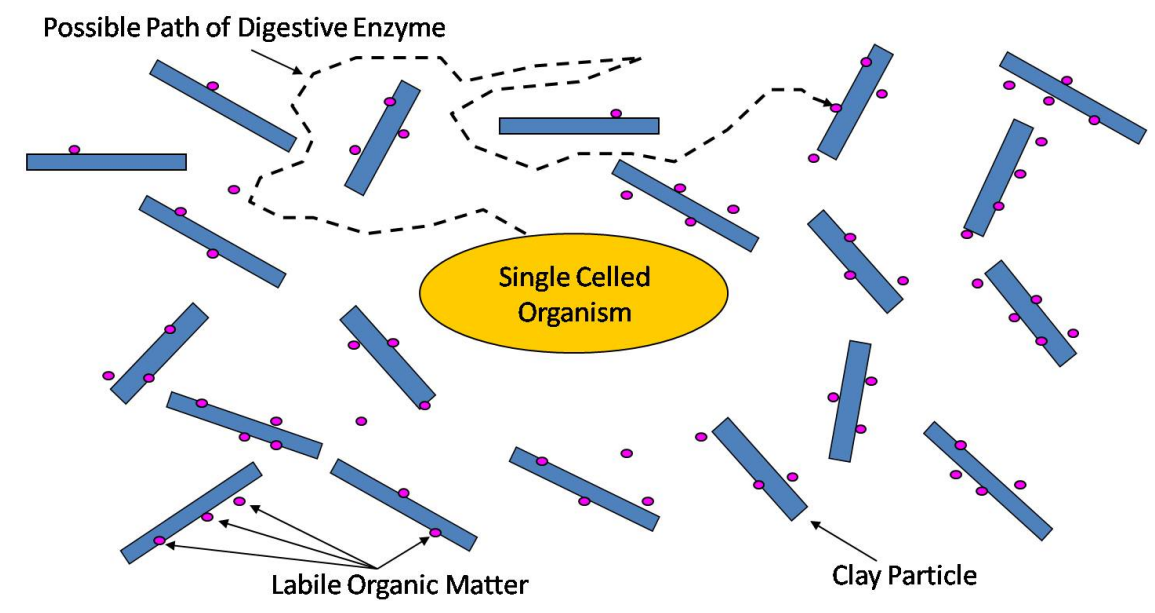

Figure 5-1: Conceptual picture of organic matter reacting in sediments. Clay particles (blue) impede the flow of enzymes from source (yellow) to organic matter (pink).

matrix [95]. In order for hydrolysis to occur, enzymes diffuse from the microbe and encounter surface associated organic matter. The local reaction rate constant $k$ is assumed to be proportional to the concentration $c$ of the enzyme. Enzymes diffuse with diffusivity $D$ and become inactive at a rate constant $\alpha$. The volume averaged enzyme concentration $\bar{c}=\phi c$ satisfies the reaction diffusion equation

$$
\frac{\partial \bar{c}}{\partial t}=\bar{D} \nabla^{2} \bar{c}-\alpha \bar{c}
$$

where $\bar{D}<D$ is the effective diffusivity which can be influenced by porosity, reversible sorption, etc [40]. For a steady supply of enzymes, one finds the approximate solution $e^{-\beta r} / r$ where $r$ is the distance from the closest microbe. If hydrolysis is diffusionlimited the characteristic distance $\beta^{-1}=\sqrt{\bar{D} / \alpha}$ an enzyme travels in a time $\alpha^{-1}$ is much smaller than the distance $r_{b}$ between microbes. Then the decay $g(t)$ of organic matter can be expressed as a superposition of exponential decays $e^{-k t}$ weighted by the probability $p(k)$ that individual parcels of organic matter react with reactivity 
$k \propto c$ yielding the approximate decay

$$
\frac{g(t)}{g_{o}}=\int_{0}^{\infty} p(k) e^{-k t} d k \sim \int_{k_{\min }}^{k_{\max }} \frac{1}{k} e^{-k t} d k \sim-\gamma-\ln k_{\min } t
$$

for $k_{\max }^{-1}<<t<<k_{\min }^{-1}[40]$. The rates $k_{\min }$ and $k_{\max }$ are the minimum and maximum attainable reaction rates which correspond to the furthest and closest that organic material can be from an enzyme source. The parameters $g_{o}$ and $\gamma$ are a normalization constant and a numerical constant, respectively. Data rescaled from sediment cores fit the theoretical curve well [40]. Physically, organic material close to the enzyme source has the highest reaction rate and disappears before organic material further from the enzyme source. Just as in the intrinsic reactivity model, rapid disappearance of material with high reaction rates leads to a slowdown of the bulk reaction rate and thus sub-exponential decay.

There are two ways of moving forward in order to determine whether physical protection or chemical heterogeneity (or a mixture of both) is the dominant process controling the decay and preservation of organic material in sediments. Experimentally, we can attempt to measure the diffusion coefficients of enzymes along with their deactivation rates in sediments. If successful, we could directly support or refute the physical protection hypothesis [40]. Additionally, we could use the theory as constructed to predict additional properties of organic matter in sedimentary systems.

\subsubsection{Linearity Between Organic Matter and Mineral Surface Area}

In addition to the continual slowdown in reaction rates, the remarkable correlation between surface area and organic carbon in sediments can be expressed in terms of impedance based models like the one expressed above [4]. The concentration profile of the enzymes, $C(r)$ as a function of the radius $r$ away from the microbe in the 
Rothman-Forney model can be expressed as

$$
C(r)=C_{1} \frac{e^{-\beta r}}{r}
$$

where $\beta=\sqrt{\alpha / D}$ is a length scale defined as the square root of the deactivation constant of the enzyme, $\alpha$, and its diffusion coefficient $D$. The RF model assumes that the rate of organic liberation, $F$, is proportional to the enzyme concentration, SO

$$
F \propto \int_{r_{0}}^{\infty} 4 \pi r^{2} \frac{e^{-\beta r}}{r} d r
$$

where $r_{0}$ is the radius of the microbe. In the limit as $r_{0} \rightarrow 0$ this can be solved to yield

$$
F \propto \frac{e^{-\beta r_{0}}\left(\beta r_{0}+1\right)}{\beta^{2}}=\frac{1}{\beta^{2}}
$$

This can be written in terms of $D$ and $\alpha$ as

$$
F \propto D / \alpha
$$

Here, we make the following maneuver. Given a fixed porosity, $\phi$, the bulk diffusion coefficient should scale inversely with surface area, $\nu$. This allows us to write the flux in terms of the surface area as

$$
F(\nu) \propto \frac{D}{\nu \alpha}
$$

To gain some understanding we wish to unpack the proportionality constant. To do this we note that the flux of organic carbon should depend on the density of organic carbon, $T_{o c}$, and a multiplicative constant, $\Psi$,

$$
F=\Psi T_{o c} \frac{D}{\nu \alpha}
$$


In this way we have an equation for the flux of organic material in the RF model which has the simplest possible dependence on the physical environment. Now, we claim that in a given chemical environment, there is a critical flux, $F_{c}$, below which a microbe will not survive. If sediments relax to this critical value, then we can solve for the total organic carbon in terms of the surface area,

$$
T_{o c}=\frac{\Psi F_{c} \alpha}{D} \nu
$$

This equation predicts a linear relationship between organic carbon percentage and surface area. The slope of this line will depend on the chemical environment.

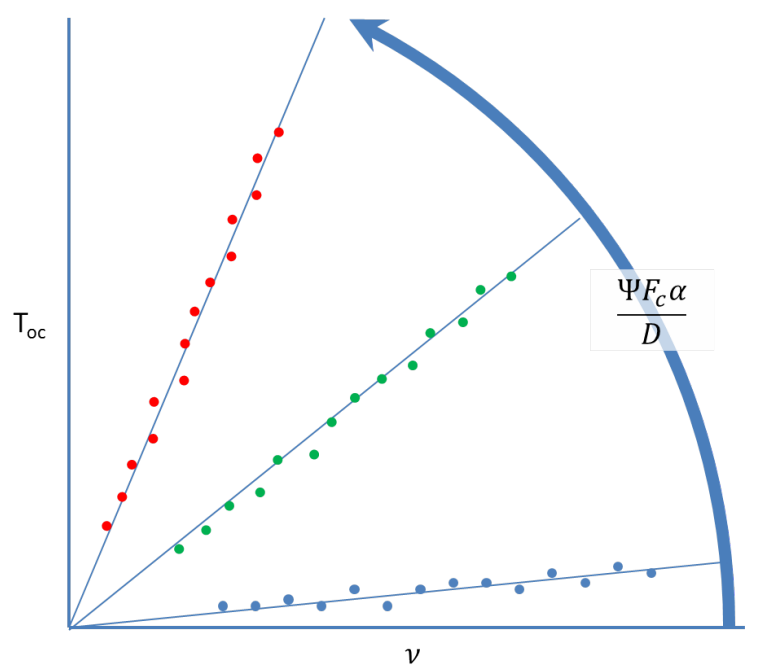

Figure 5-2: This is a schematic diagram of how the theory should manifest itself in sedimentary data. The progression from blue to red dots is the chemical progression from deep ocean oxic, to shelf, to anoxic depositional environments.

\subsubsection{Power-law Dependence and Organism Density}

Microbial density $D$ seems to follow a power law distribution with depth $D \propto d^{-\gamma}$ where $d$ is the depth in the sediment and $\gamma$ is in the range from $.3-1.2$ [112]. If one assumes that the first order decay rate is also a power law of the form $k=\frac{-\alpha}{t}$. 
Ideally, one would continue to extend the above formulation for the organism density. However, this seems difficult, so instead I will consider the Michaelis-Menten approximation [113]

$$
R_{i}=V_{\max } \frac{[S]}{K_{M}+[S]}
$$

for the rate at which a given individual organism $R_{i}$, consumes a resource $S$ at a given concentration $[S]$,(brackets indicate concentration). $V_{\max }$ is the maximum consumption rate by an individual and $K_{M}$ is a constant which is the value of $[S]$ where $R_{i}$ is half its maximum attainable value. This is the first approximation one can make for the effects of resource limitation on an organism.

We now return to the solution for decay in sediment. If $k=\frac{-\alpha}{t}$ then we can use the fact that $[\dot{S}]=k[S]$ to write $[\dot{S}]=\frac{-\alpha}{t}[S]$. Rearranging this equation and integrating then gives us

$$
\ln \frac{[S]}{\left[S_{o}\right]}=\ln \left(\frac{t}{t_{o}}\right)^{-\alpha}
$$

where the subscript o denotes the initial time. This can be further simplified to

$$
[S]=\left(\left[S_{o}\right] t_{o}^{\alpha}\right) t^{-\alpha}
$$

Multiplying both sides of equation (5.11) with the number density $d$ of organisms and using the relation that $R_{i} * d=-[\dot{S}]$ yields

$$
R_{i} * d=-[\dot{S}]=V_{\max } \frac{[S] d}{K_{M}+[S]}
$$

This can be rearranged to give an expression for $d$ as

$$
d=-\frac{[\dot{S}]\left(K_{M}+[S]\right)}{V_{\max }[S]} .
$$

In our case, the organismal community is acting way below maximum values, so it 
seems like a reasonable approximation to make that $K_{M}>>[S]$. We now write

$$
d \approx-\frac{K_{M}[\dot{S}]}{V_{\max }[S]}
$$

and substitute for $[S]$ with equation (5.13) to find

$$
d \approx \frac{\alpha * K_{M}}{V_{\max }} t^{-1}
$$

or

$$
d \propto t^{-1}
$$

If we invoke that $t \propto D$ where $D$ is depth in sediment then we now have a relationship between $d$ and $D$ namely that $d \propto D^{-1}$.

\subsubsection{A Physical-Reactive Continuum}

The unification of the slowdown in decay rate with the correlations between organic matter and mineral surface area, as well as the possible extension to bacterial counts provide real motivation behind the physical-reactive continuum as a potentially important factor in the processing of organic material in the environment. If our assertion that surface area acts as an impedance to the agents of degradation, rather than a direct protector of associated organic material, then we have a conceptual framework to explain additional processes like the presumed stripping of organic material from particles upon being transported from river systems into the ocean.

If the originally posed mechanisms of physical protection were correct [95] then we would expect that organic material aggregated with mineral surfaces in river systems would remain protected. However, this does not appear to be the case. In outflow to the Amazon river system, for example, organic particles seem to be effectively stripped of organic material. If the association with particles is protective because of 


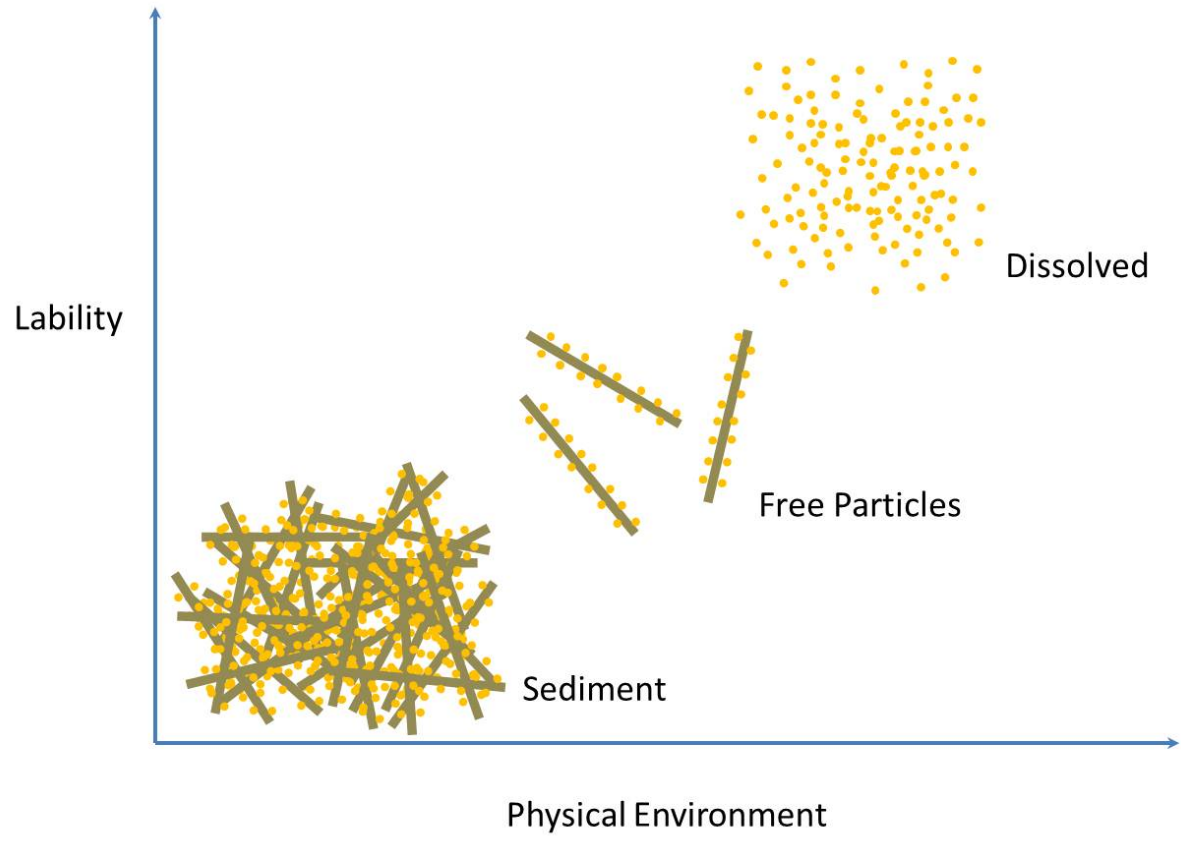

Figure 5-3: This is a schematic diagram of how the physically mediated reaction rate might change with the physical environment.

their impedance properties, this can be effectively understood in terms of an axis of lability versus physical environment (see Fig.5-3).

The protective power of particles in this formulation is due to the complex, chemically active environment they create. In a static sediment with no flow this power is maximized. If the sediment is continually turned over as in the Amazon delta, we would expect the bulk decay rate to increase as organic material had better access to oxidizing agents and organisms.

\subsection{Experimentally Testing Diffusion Limitation in Sediments}

If the tenets of the RF model were to hold they form a powerful conceptual unification of organo-sedimentary processes. The RF model has been challenged, however, 
on two grounds [114]. First, Boudreau et al. dispute the assumption of diffusion limitation, i.e. that $\beta r_{b}>>1$. This condition leads to a wide range of enzyme concentrations in space and thus a wide range of reaction rates. Invoking a bulk diffusion coefficient for moderately sized molecules in solution of $10^{-6} \mathrm{~cm}^{2} \mathrm{~s}^{-1}$, and a value for $\alpha$ of $10^{-2}$ to $10^{-6} \mathrm{~s}^{-1}$ derived from experimental observations of enzymatic lifetimes in solution, Boudreau et al. suggest values of $\beta$ which are much smaller than those required by the $\mathrm{RF}$ model. Instead of an environment dominated by heterogeneity, Boudreau et al. invoke a well-mixed sedimentary regime where degradative enzymes are homogeneously distributed. However, the experiments cited by Boudreau et al. did not measure $\beta$ directly. Enzymatic lifetimes were inferred from experimental observations of the period over which enzymes retain their catalytic activity in solutions overlaying sediment, while the diffusion coefficient was modeled from lipid data. We believe that values for enzymatic lifetimes in solutions overlaying sediments are probably not representative of $\alpha$ in sediment and that sorptive processes have the potential to influence the diffusive properties of enzymes and thus $\alpha$ and $\beta[110,115]$. As enzymes diffuse through a surface active mineral matrix, they encounter and become affixed to surfaces with a certain probability. This diffusion with trapping could alter the observed diffusion coefficient by several orders of magnitude. The encapsulation of organic material by clay particles (shielding) could also have a strong influence on degradation [108]. While some sediment could be readily accessible to enzymes and have one characteristic $\beta$, other organic matter may be surrounded by minerals, reducing the local value of $\beta$ well above values measured in solutions. The geometries of organo-clay aggregates could therefore drastically influence degradation rates of organic matter. Boureau et al. also assert that the intrinsic chemical heterogeneity of organic matter is the dominant mechanism behind its degradation dynamics. Although this may be true for certain environments, there is strong evidence that the lability of organic carbon in sediments stays constant over long timescales [93]. 
Both physical (RF model) and chemical (chemical heterogeneity model) factors could regulate organic matter decay. However, unlike the chemical heterogeneity model, the assumptions of the RF model are theoretically amenable to direct experimental measurement. Microbes in sediment release extra-cellular enzymes which travel on random walks to react with organic matter on particle boundaries [40]. The lysate diffuses back to the organism in what can be a successful feeding strategy [115] as demonstrated in experiments [25].

In order to have diffusion limitation, we require either a low diffusion coefficient, a fast deactivation rate, or both. On a first pass, we can calculate the bulk diffusion coefficient as a function of the bulk porosity, the molecular size, and the liquid-solid partition coefficient. The expected diffusion coefficient for enzymes in free solution is $10^{-7}-10^{-6} \mathrm{~cm}^{2} / \mathrm{s}[115]$. We assume a sediment porosity near .5 and a deactivation rate which is likely less than a few hours $\left(\alpha \approx 10^{-4}\right)[115,116]$. The partition coefficient of these enzymes is unclear, but proteins have partition coefficients $\sim 100-1000$ in marine sediments [117]. The RF model suggests that $\beta * r \approx 5$, where $r$ is the characteristic distance between microbes. A partition coefficient of .001, a porosity of $.5, \alpha \approx 10^{-4}$, and a characteristic distance of $10 \mu \mathrm{m}$ between microbes yields an acceptable value of $\beta * r \approx 1.4$ which could be larger if the microbial spacing is underestimated [4].

In lieu of better data, the proper course is to directly measure both the effective diffusion coefficient, $\bar{D}$ and the deactivation rate $\alpha$ inside the sedimentary matrix. We have used both nmr and HPLC methods in our attempt to measure $\bar{D}$ and have attempted to build fluorogenic tracers to directly measure the spacially localized hydrolysis rates. Both the nmr method and the HPLC method have proven capable of measuring diffusion coefficients, but we have yet to find a way of measuring them at the proper concentration and timescale. The fluorogenic rate tracers were not successful. What follows is an explanation and discussion of these three techniques 


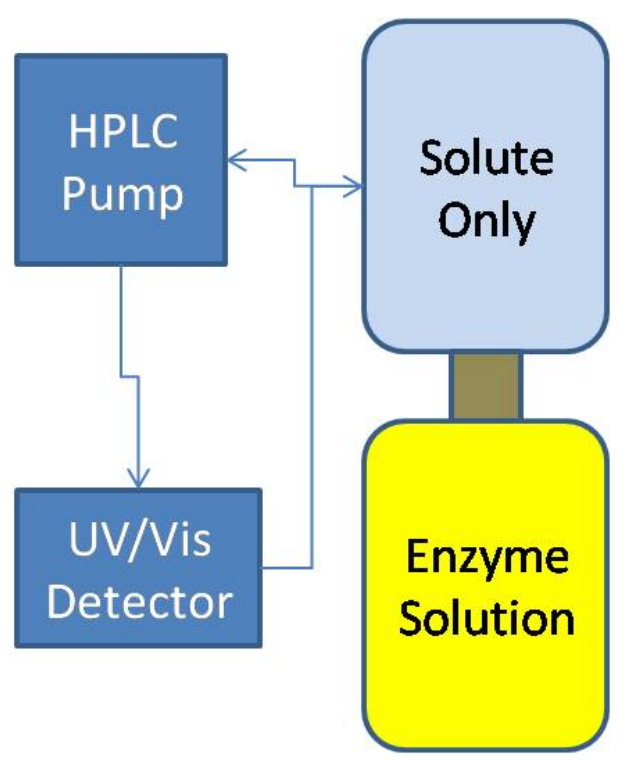

Figure 5-4: This is a schematic of a diffusion cell we will use for measuring the diffusion coefficients of enzymes in sediment. Enzyme starts at a high concentration (yellow bottle) and diffuses through the sediment plug (brown box) and into the bottle containing only solute. The concentration in the upper bottle is measured by pumping fluid through a UV/Vis detector.

and what might be done in the future to fully test the RF model.

\subsubsection{Measuring the Effective Diffusion Coefficient in Porous Media}

Geometric control over organic matter decay requires diffusion limitation or that $\sqrt{\bar{D} / \alpha}<<r_{b}$. This can be tested by directly measuring the bulk diffusion coefficient of enzymes in sediment in conjunction with their activity. There are many conceptual methods of measuring diffusion, but the most common method is to use techniques based on diffusion cells [118]. A simple schematic of such a diffusion cell is outlined in figure 5-4. In our case, we desire a system which allows for not only the measurement of diffusion coefficients, but could be expanded to look at the distribution of diffusion coefficients due to the complex micro-environments found in sedimentary environments. Towards this objective, we decided to explore conducting the diffusion 
measurements in situ using pulsed field gradient techniques.

\subsection{Measuring Diffusion Through NMR}

Diffusion cell measurements suffer from the time required to run them and the bias induced by using bulk concentration changes to infer the diffusion coefficient. Ideally, one would measure the diffusion of molecules in situ while they were inside the sediment. This can be done using a technique call pulsed field gradient spin echo NMR spectroscopy (PFGSE) [118-120], which can in principle measure diffusion coefficients as low as $10^{-11} \mathrm{~cm}^{2} \mathrm{~s}^{-1}$. In addition, it can directly probe the distribution of diffusion coefficients inside a media.

The premise of the technique is based on standard re-phasing in spin echo NMR. The magnetic moments, $\mu_{i}$, of the nuclei of interest are aligned in the $\hat{z}$ direction with a strong, uniform magnetic field $B_{o}$. Fluctuations in the bulk magnetization of the material, $M=\sum \mu_{i}$, are what is measured by the NMR spectrometer. At the beginning of the experiment, $M$ is aligned in the same direction as $B_{o}$. At this point, an oscillating magnetic field, $B_{1}$, is used to rotate the magnetic moment vector, $M$ by $\frac{\pi}{2}$ radians. The individual magnetic dipoles now act as spinning tops in a gravitational field, and process in phase around the $\hat{z}$ axis at their natural frequency which is a function of $B_{o}$. The sample is then exposed to a magnetic field gradient in the $\hat{z}$ direction. This de-phases the individual $\mu_{i}$ as it exposes different nuclei to varying magnetic field strengths. After a given time, $M$ is exposed to another oscillating field which rotates $M$ in the opposite direction by $\pi$. The sample is then exposed to the same gradient field as previously for the same time duration. If all of the molecules remain stationary throughout the entire pulse sequence then the two gradient pulses should have equal and opposite phasing effects. In this case the NMR spectrometer should measure a signal which does not depend on the time duration between the 


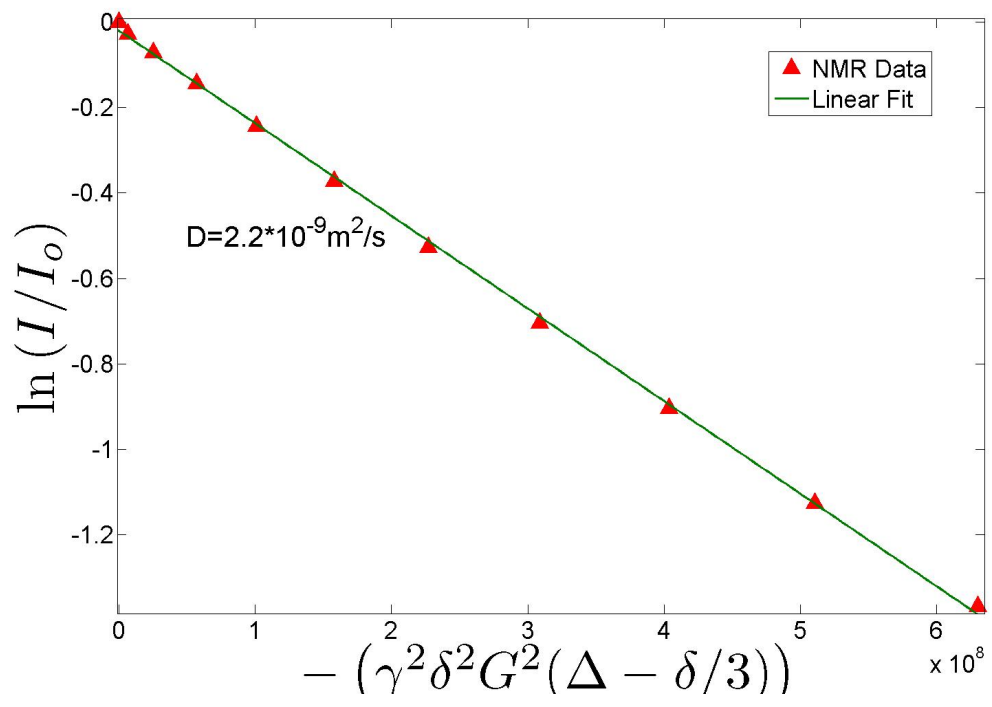

Figure 5-5: By changing the gradient strength we can find the diffusion coefficient by finding the negative of the slope on the above figure. We find the diffusion coefficient of water in itself to be $2.2 * 10^{-9} \mathrm{~m}^{2} / \mathrm{s}$.

first and second gradient pulses. If molecular diffusion does take place, then it can be measured by how the signal intensity changes with the time delay between pulses. Formally, this is expressed by the equation [121]

$$
A=A_{o} e^{-\left(\gamma \delta g_{c} I\right) D(\Delta-\delta / 3)}
$$

where $A_{o}$ is the signal under no diffusion, $\gamma$ is the gyromagnetic ratio, $\delta$ is the duration of the magnetic field gradient pulses, $g_{c}$ is the magnitude of the magnetic field gradient, $I$ is the amplitude of the current pulsed through the gradient coil, $D$ is the diffusion coefficient, and $\Delta$ is the time between the two gradient pulses. By measuring the amplitude $A$ as a function of $g_{c}$ or $\Delta$ it becomes possible to measure the bulk diffusion coefficient [120-122]. We demonstrated feasibility on our NMR spectrometer by measuring the diffusion coefficient of water (Fig. 5-5). This is possible for liquid solvent, porous media, and even solid systems although complications exist because of the inherent magnetic inhomogeneity of most porous systems. Unfortunately, ar- 


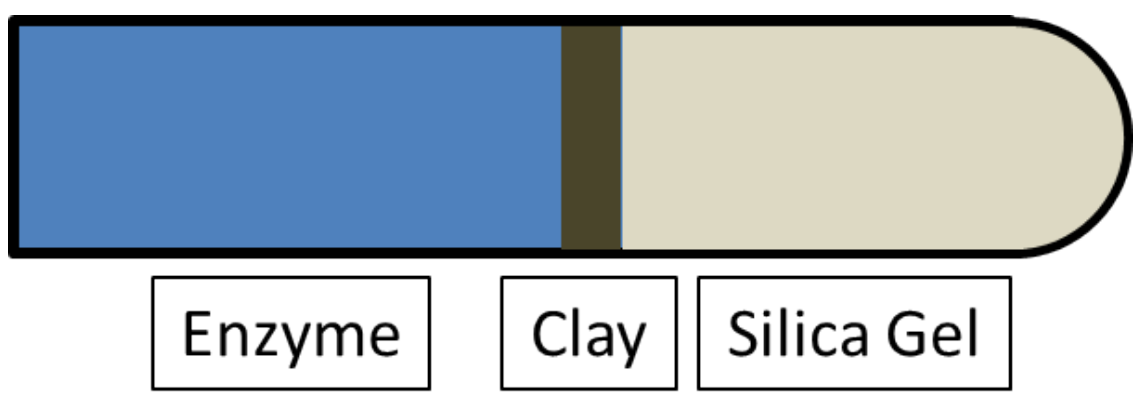

Figure 5-6: A rotated view of the nmr diffusion cell. The compound of interest diffuses through the clay layer and into the silica gel where it is observed by the nmr. In reality the layer labeled 'enzyme' is substantially longer.

tificial sediments consisting of deuterated water, water, and clay produced no nmr signal. This is most likely due to potential iron impurities in the clay substrate, but the reason is not entirely clear. Strategies for dealing with these issues are to dilute the substrate with silica gel, move to solid state nmr, or use complex pulse programs which actively damp the feromagnetic hinderance of iron.

In order to access our primary objective of measuring the diffusion coefficient through a sediment matrix we continued using nmr methods, but turned them into something akin to a diffusion cell. Although we were unable to get an nmr signal from clay slurries, we were successful in measuring broad spectra of single compounds in silica gel for liquid chromatography (10 $\mu \mathrm{m}$ bead size). We utilized this to generate a column where the measurement region is silica gel, capped by a region of clay substrate. On top of the clay substrate is placed a solution containing the compound of interest. Initially, there is no nmr signal due to the compound. Over time, the compound diffuses through the clay and into the silica where it's broad peaks can be measured. A rotated view of the system can be seen in figure 5-6. Building this setup is straightforward. A silica gel slurry is made and placed in an nmr tube. The tube is then centrifuged to get a nice and compact layer. The supernatant is removed and a clay slurry is added. This is again centrifuged. The final step is to remove the supernatant again before adding the enzyme to the top of the column. This final 
step is done right before measurement. One could use this system in the same way as the diffusion cell expressed above. However, as a simple measurement of the diffusion coefficient a measurement of the time between the start of the experiment and when the compound is first detected yields a reasonable estimate. Two compounds were run in this fashion: $\alpha$-amylase and alanine. The compound $\alpha$-amylase has a diffusion coefficient at STP of $D=8 * 10^{-7} \frac{\mathrm{cm}^{2}}{\mathrm{~s}}$ using both the equation from above and found by measurment [123]. In our experiment, the clay plug was approximately $l=3 \mathrm{~mm}$ long. The length can be written as a function of the penetration time, $t_{p}$, and the effective diffusion coefficient $\bar{D}$ as

$$
l=2 \sqrt{D t_{p}}
$$

With a measured penetration time of 20 hours we estimate the effective diffusion coefficient of $\alpha$-amylase to be $3 * 10^{-7} \frac{\mathrm{cm}^{2}}{\mathrm{~s}}$. This is consistent with the porosity of our clay plug potentially augmented by a slight partitioning between the clay and the water. For amylase, we found a penetration time of 8 hours which gives an estimated diffusion coefficient of $8 * 10^{-7} \frac{\mathrm{cm}^{2}}{\mathrm{~s}}$. The expected free diffusion coefficient for this compound is $D=9 * 10^{-6} \frac{\mathrm{cm}^{2}}{\mathrm{~s}}$, or approximately ten times what we calculate the effective value to be. This requires a solid-liquid partition coefficient in our system near 20, a value consistent with partition values of other amino-acids on clays [124]. What is confusing is why the substantially larger enzyme would not partition more instead of less.

This line of experimentation seems promising. One potential issue is the high concentrations of solute used in the amylase experiment $(50 \mathrm{mg} / \mathrm{mL})$. It is possible that at such high concentrations our clay plug is rapidly saturated by the large enzyme, allowing diffusion to continue unhampered. In order to use lower, more naturally relevant concentrations, the system should be shifted, so that the diffusion moves 


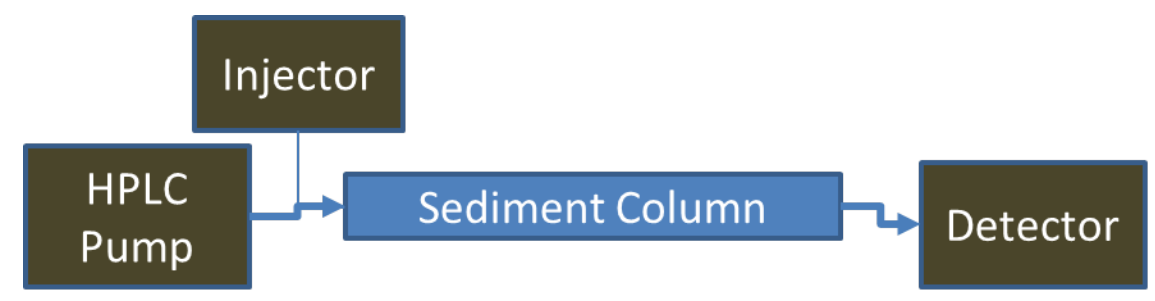

Figure 5-7: This is a schematic of the HPLC apparatus used to measure the diffusion of simple compounds. The only modification is the 'Sediment Column' in place of HPLC chromatography columns. For our simple proof of concept experiments we used standard C18 media.

enzyme into the free solution. In this case the sensitivity and signal to noise ratio should greatly increase, allowing a more relevant concentration range to be explored. Additionally, the use of observation nuclei other than hydrogen may help open up the other nmr-based methods. PFGSE experiments in systems like cement have been successful using nuclei like sodium and fluorine which had a much greater signal to noise in complex environments. In addition to this nmr setup, we explored using an HPLC based approach to accomplish the same objectives.

\subsection{Measuring Diffusion Through HPLC Methods}

If we can pack the porous material of interest into a column, then we can use the machinery behind liquid chromatography (HPLC) to measure the effective diffusion coefficient of compounds which interest us. The basic liquid chromatography system (Fig. 5-7) can be used as is to measure the diffusion coefficient of any compound of interest.

The method is straightforward. The compound of interest is injected onto the column and pumped through until it is detected at the detector at $t_{r}$. At this point, we repeat the previous experiment with a slight modification. The substance is injected onto the column and pumped through for half of the retention time. At this point, the pump is stopped and the system is allowed to rest for a time $\Delta t$ before the 
pump is re-started and the substance is pumped out past the detector. Ignoring the peak shape created under normal HPLC conditions, or assuming that it emerges as a single discrete peak, we would expect the detector function to emerge as a Normal distribution of the form

$$
C(t)=\frac{1}{\sqrt{4 \pi D \Delta t}} e^{-\frac{\gamma t}{4 D \Delta t}}
$$

Here, we set $t^{\prime}=0$ as the time at which the peak of the distribution reaches the detector. The multiplicative constant $\gamma$ in front of $t$ converts time into linear distance through the flow volume, porosity of the material, and dimensions of the column.

As diffusion is a linear process, the function that we measure coming out of the detector, $F(t)$, is a convolution of the instrument function, $I(t)$, with the idealized diffusion solution, $C(t)$,

$$
F(t)=\int_{-\infty}^{\infty} I\left(t-t^{\prime}\right) C(t) d t^{\prime}
$$

In general, using this method would require a numerical deconvolution based on the instrument function for $\Delta t=0$. However, the detector function in this case tends to be a normal distribution. The variance of two convolved normal distributions is also a normal distribution where the variance is the sum of the variances of the parent distributions. In this case, we would expect a plot of the variance as a function of $\Delta t$ to yield a straight line with a slope equal to twice the diffusion coefficient.

This experiment was done effectively using Glycine as the test compound. The experiment was done multiple times for each $\Delta t$ and the results can be seen in figure 5-8. Unfortunately, when enzymes were tried in this system they became affixed to the column and would not move unless organic solvents were added. The fundamental issue with these experiments the way they are currently constructed is based on the inverse correlation between the diffusion coefficient and the retention time. Compounds with very small diffusion coefficients based on their partition coefficients will have extremely long retention times on the column which are outside our ability to 

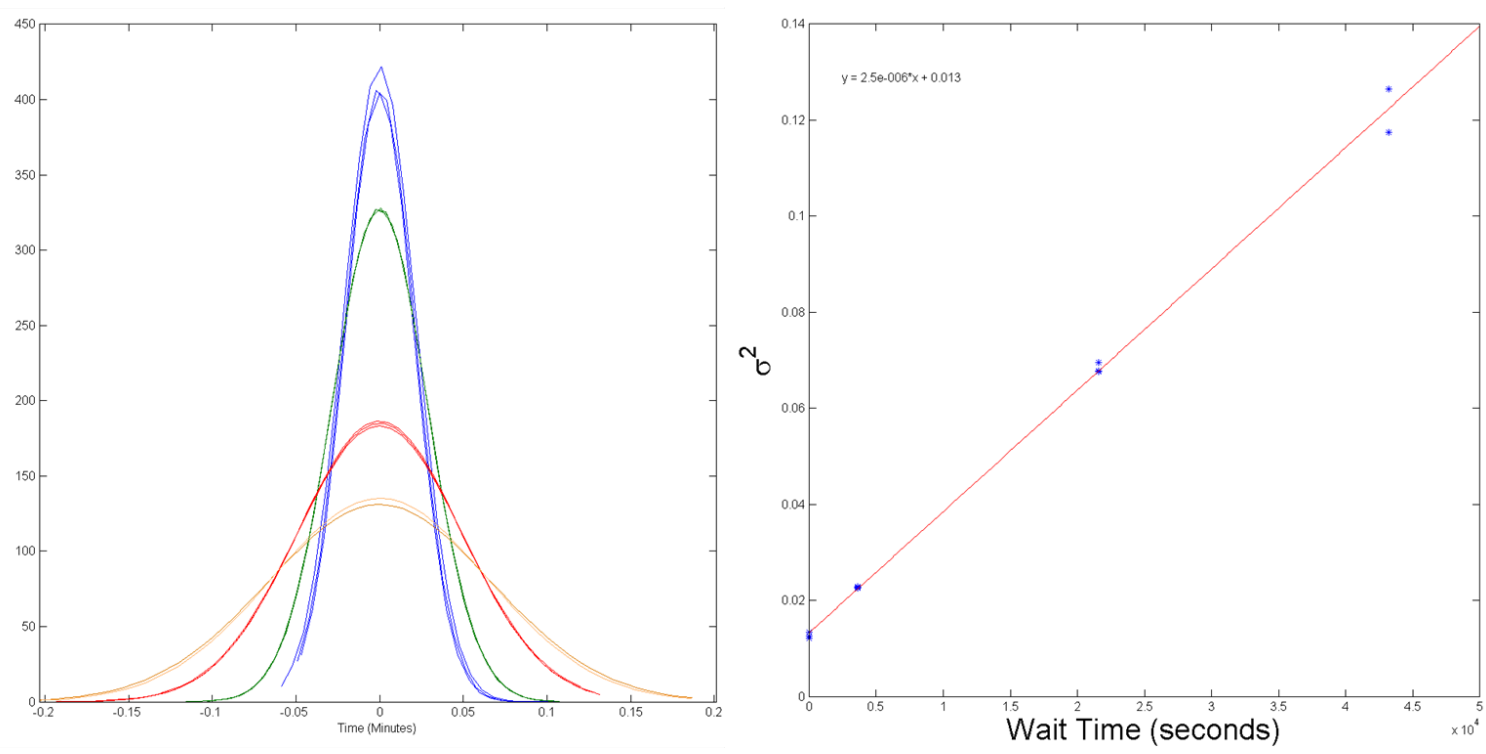

Figure 5-8: These plots are used to calculate the bulk diffusion of Glycine in the column. (Left) This is a plot of the relative intensity at the HPLC detector for different wait times $(\Delta t)$. The times are 1 minute, 1 hour, 6 hours, and 12 hours for the blue, green, red, and orange curves respectively. As the wait time increases, the hight of the peak decreases and the variance increases. (right) This is a plot of the measured variance as a function of the wait times in the HPLC apparatus. The linearity suggests consistency with diffusion, while the intercept represents the gaussian instrument function. 
measure effectively. Despite these issues, we consider this simple system as having future potential with perhaps small modifications.

\section{Direct Modeling of 2D HPLC System}

HPLC and the simple diffusion experiment conducted above work because the microscale fluctuations in concentration, porosity, and partitioning average out when one considers many hundreds of pores. There are good questions, however, as to how this upscaleing occurs and under what conditions it breaks down. We used a previously solved flow-field of fluid flow through a two dimensional bed of impermeable particles across hundreds of pore widths as a numerical template for computationally modeling the above experiment.

As an initial test, we treated our enzyme as a concentration field and let a pulse of material advect through the particle bed. Akin to the experiment, we stopped in the middle, and then continued after waiting a fixed amount of time. For computational reasons, we modified the diffusion coefficient and kept $\Delta t$ the same. We were able to generate the same kind of progressively wider Gaussians with a reasonable relationship between the variance and the diffusion coefficient (see Fig. 5-9).

The current formulation has a few issues based mostly on the finite length of the bead bed which is substantially smaller than in the experiment. This causes both the kink in the variance plot and the deviation from gaussian behavior. Despite this issue, this system is simple, and has a nice analogous experiment. Current outstanding problems are a result of the difficulty in modeling a partially absorbing boundary which can both store and release enzyme. A better computational model of the experimental system helps both our theoretical understanding and our experimental design. 


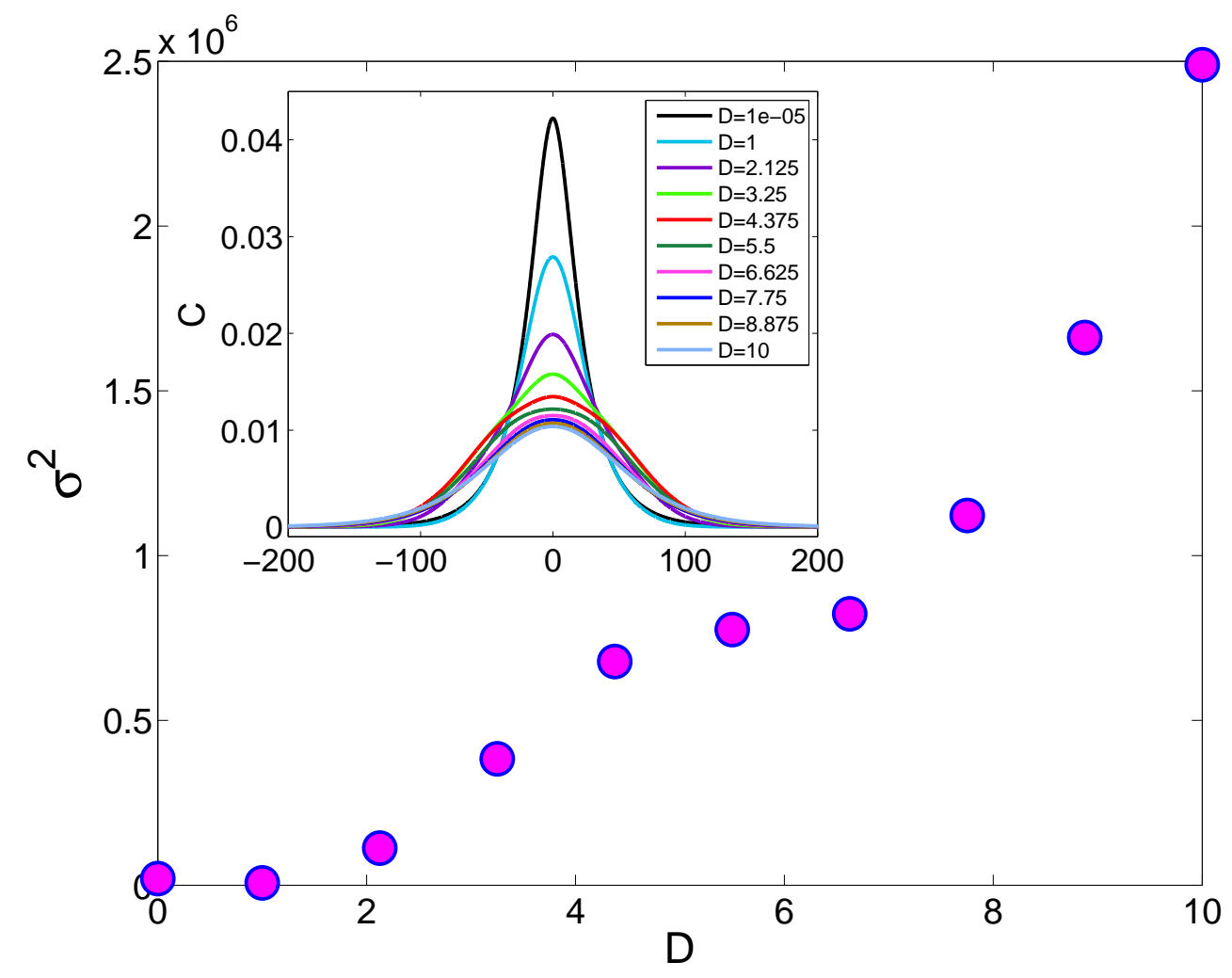

Figure 5-9: These plots are used to calculate the bulk diffusion of Glycine in the column. (Left) This is a plot of the relative intensity at the HPLC detector for different wait times $(\Delta t)$. The times are 1 minute, 1 hour, 6 hours, and 12 hours for the blue, green, red, and orange curves respectively. As the wait time increases, the hight of the peak decreases and the variance increases. (right) This is a plot of the measured variance as a function of the wait times in the HPLC apparatus. The linearity suggests consistency with diffusion, while the intercept represents the gaussian instrument function. 


\subsection{Final Thoughts}

The complexity of decaying particulate organic material is not in question. With millions of compounds and a complex physical environment, it is no great surprise that organic carbon is not remineralized with a single, characteristic rate constant [104]. What is surprising, is that a measure of the apparent rate constant follows a power law behavior accross over ten-orders of magnitude in time. Is this behavior due to some kind of order that arises from the complexity of the environment? In the RF model, the unifying characteristic is diffusion limitation, while in other formulations [3] chemistry relaxes to the proper initial distribution. We lead additional credence to the RF formulation by understanding mineral surface area as acting like an impedance. Using this concept, we predict the linearity between organic carbon and mineral surface area. Because the impedance idea does not require any specific form of mineral association, it fits naturally into a framework of a physical-reactive continuum. Minerals have the greatest effect in stagnant environments. Moving forward, diffusion limitation requires formal experimental testing, potentially of the type attempted here. Only through the creation of simple experimental model systems will we be able to fully test the ideas of physically mediated protection and chemical heterogeneity. 


\section{Chapter 6}

\section{Parting Thoughts}

Organic carbon cycling in the ocean is a complex system which will require both further advancement in methodology and theory before we can be confident in our models of its current and ever changing state. In systems that become more and more complex approaching the microscale, there is always promise that what appears complicated can be unified by thinking about the system in terms of the proper turnover time distributions and their evolution. When averaging over the microscale and assuming that a carbon reservoir can be treated as such we make implicit assumptions as to the underlying age and turnover time distributions. Depending on the nature of these distributions, bulk properties of the network like carbon flux can vary between extremes. These assumptions are especially important to consider when using isotope methods as the measure of the average age and turnover time.

In an attempt to uncover a single turnover time distribution for deep water DOC, we instead provided evidence for a different theory for this carbon reservoir. We found evidence from both novel spectrometric techniques and bulk DOC data that the deep ocean DOC contains two widely separated groups of material. One subreservoir has a radiocarbon age substantially older than currently believed, while the other appears to be modern. We suggest that this modern carbon comes from sinking 
particles. This reservoir of material is consistent with microbial metabolism at depth and can be used to explain the anomalous aging of DOC in the deep ocean. Here, the combination of theory with experiment leads to novel and relevant insights into the carbon cycle.

A similar attempt was made to bring novel experimental techniques to bear on the problem of organic matter decay in sediments, but with far less success. Although both the nmr technique and the HPLC method for measuring diffusion coefficients may bear fruit in the future, we were unable to provide the kind of experimental verification we intended. Theoretical progress was made by thinking about surface area in sediments in terms of an impedence. We were able to combine physical models for organic matter preservation with the linear correlations found between mineral surface area and organic carbon content in sediments.

The oceanic carbon cycle contains enough complexity to commit the attention of researchers for many years to come. When one adds to this the increasing burden of climate change science, the questions are almost endless. One method for dealing with this complexity is to apply a theoretical framework towards dealing with smaller and smaller scales. This thesis took one step forward and found success in a further understanding of the cycling of deep ocean DOC. 


\section{Bibliography}

[1] S.R. Emerson and J.I. Hedges. Chemical oceanography and the marine carbon cycle. Cambridge University Press, 2008.

[2] T. Dittmar. The molecular level determination of black carbon in marine dissolved organic matter. Organic Geochemistry, 39(4):396-407, 2008.

[3] B. P. Boudreau and B. R. Ruddick. On a reactive continuum representation of organic matter diagenesis. American Journal of Science, 291:507-538, 1991.

[4] D.H. Rothman and D.C. Forney. Physical Model for the Decay and Preservation of Marine Organic Carbon. Science, 316(5829):1325, 2007.

[5] David C Forney and Daniel H Rothman. Common structure in the heterogeneity of plant-matter decay. Journal of The Royal Society Interface, 9(74):2255-2267, 2012.

[6] J.J. Middelburg. A simple rate model for organic matter decomposition in marine sediments. Geochim. Cosmochim. Acta, 53(7):1577, 1989.

[7] H. Von Foerster. Some remarks on changing populations in the kinetics of cellular proliferation, frederick stohlman, jr. New York, Grune and Stratton, pages 382-407, 1959.

[8] P.M. Williams and E.R.M. Druffel. Radiocarbon in dissolved organic matter in the central north pacific ocean. Nature, 330(6145):246-248, 1987.

[9] S.R. Beaupré and E.R.M. Druffel. Constraining the propagation of bombradiocarbon through the dissolved organic carbon (doc) pool in the northeast pacific ocean. Deep Sea Research Part I: Oceanographic Research Papers, 56(10):1717-1726, 2009.

[10] S.R. Beaupré and L. Aluwihare. Constraining the 2-component model of marine dissolved organic radiocarbon. Deep Sea Research Part II: Topical Studies in Oceanography, 57(16):1494-1503, 2010.

[11] B. P. Boudreau and B. R. Ruddick. On a reactive continuum representation of organic matter diagenesis. American Journal of Science, 291:507-538, 1991. 
[12] E.R.M. Druffel, P.M. Williams, J.E. Bauer, and J.R. Ertel. Cycling of dissolved and particulate organic matter in the open ocean. Journal of Geophysical Research, 97(C10):15639-15, 1992.

[13] D.A. Hansell, C.A. Carlson, and R. Schlitzer. Net removal of major marine dissolved organic carbon fractions in the subsurface ocean. Global Biogeochemical Cycles, 26(1):GB1016, 2012.

[14] Dennis A Hansell. Recalcitrant dissolved organic carbon fractions. Annual Review of Marine Science, 5:421-445, 2013.

[15] D.C. Smith, M. Simon, A.L. Alldredge, and F. Azam. Intense hydrolytic enzyme activity on marine aggregates and implications for rapid particle dissolution. Nature, 359(6391):139-142, 1992.

[16] D.A. Hansell, C.A. Carlson, D.J. Repeta, and R. Schlitzer. Dissolved organic matter in the ocean: a controversy stimulates new insights. Oceanography, $22: 32-36,2009$.

[17] Stephen O Rice. Mathematical analysis of random noise-conclusion. Bell Systems Tech. J., Volume 24, p. 46-156, 24:46-156, 1945.

[18] Paul G Falkowski, Richard T Barber, and Victor Smetacek. Biogeochemical controls and feedbacks on ocean primary production. Science, 281(5374):200206, 1998.

[19] T.P. Guilderson, D.P. Schrag, S.J. Fallon, R.B. Dunbar, K.H. Kilbourne, and N. Grumet Prouty. Surface water radiocarbon $\left(\delta^{14} \mathrm{c}\right)$ reconstructed from reefbuilding zooxanthellate corals from 1751-2004. Carbon Dioxide Information Analysis Center, 2005.

[20] B. Mortazavi and J.P. Chanton. Use of keeling plots to determine sources of dissolved organic carbon in nearshore and open ocean systems. Limnology and oceanography, pages 102-108, 2004.

[21] E. R. M. Druffel and J. E. Bauer. Radiocarbon distributions in Southern Ocean dissolved and particulate organic matter. Geophysical Research Letters, 27:1495-1498, 2000.

[22] Robert M Key, Alex Kozyr, Chris L Sabine, Kitack Lee, Rik Wanninkhof, John L Bullister, Richard A Feely, Frank J Millero, Calvin Mordy, and T-H Peng. A global ocean carbon climatology: Results from global data analysis project (glodap). Global Biogeochemical Cycles, 18(4), 2004.

[23] S.R. Beaupré, E.R.M. Druffel, and S. Griffin. A low-blank photochemical extraction system for concentration and isotopic analyses of marine dissolved organic carbon. Limnol. Oceanogr. Methods, 5:174-184, 2007. 
[24] Y. Oba and H. Naraoka. Carbon and hydrogen isotopic fractionation of low molecular weight organic compounds during ultraviolet degradation. Organic Geochemistry, 39(5):501-509, 2008.

[25] YA Vetter and JW Deming. Growth rates of marine bacterial isolates on particulate organic substrates solubilized by freely released extracellular enzymes. Microbial ecology, 37(2):86-94, 1999.

[26] R. A. Berner. Burial of organic carbon and pyrite sulfur in the modern ocean: its geochemical and environmental significance. American Journal of Science, 282:451-473, 1982.

[27] R. A. Berner and D. E. Canfield. A new model for atmospheric oxygen over phanerozoic time. American Journal of Science, 289:333-361, 1989.

[28] B Moore III and B Bolin. Oceans, carbon dioxide, and global climate change. Oceanus;(United States), 29(4), 1986.

[29] Alan R Longhurst. Role of the marine biosphere in the global carbon cycle. Limnology and Oceanography, 36(8):1507-1526, 1991.

[30] E. R. M. Druffel, P. M. Williams, J. E. Bauer, and J. R. Ertel. Cycling of dissolved and particularte organic matter in the open ocean. Journal of Geophysical Research, 97:15639-15659, 1992.

[31] D.A. Hansell and C.A. Carlson. Deep-ocean gradients in the concentration of dissolved organic carbon. Nature, 395(6699):263-266, 1998.

[32] P. G. Falkowski. The role of phytoplankton photosynthesis in global biogeochemical cycles. Photosynthesis Research, 39:235-258, 1994.

[33] J. H. Martin, G. A. Knauer, D. M. Karl, and W. W. Broenkow. VERTEX: carbon cycling in the northeast Pacific. Deep-Sea Research, 34:267-285, 1987.

[34] T. Dittmar and B.P. Koch. Thermogenic organic matter dissolved in the abyssal ocean. Marine chemistry, 102(3-4):208-217, 2006.

[35] T. Dittmar and J. Paeng. A heat-induced molecular signature in marine dissolved organic matter. Nature Geoscience, 2(3):175-179, 2009.

[36] AG McKendrick. Mathematics applied to medical problems. Roy. Soc. Edinh, 1926.

[37] SN Wood. Obtaining birth and mortality patterns from structured population trajectories. Ecological Monographs, 64(1):23-44, 1994.

[38] B. Bolin and H. Rodhe. A note on the concepts of age distribution and transit time in natural reservoirs. Tellus, 25(1):58-62, 1973. 
[39] William J Tarutis Jr. On the equivalence of the power and reactive continuum models of organic matter diagenesis. Geochimica et cosmochimica acta, 57(6):1349-1350, 1993.

[40] Daniel H Rothman and David C Forney. Physical model for the decay and preservation of marine organic carbon. Science, 316(5829):1325-1328, 2007.

[41] B.E. Rosenheim, M.B. Day, E. Domack, H. Schrum, A. Benthien, and J.M. Hayes. Antarctic sediment chronology by programmed-temperature pyrolysis: Methodology and data treatment. Geochemistry Geophysics Geosystems, 2008.

[42] SR Beaupre and ER Druffel. Uv-induced serial-oxidations of marine dissolved organic carbon (doc) and biogeochemical implications. In AGU Fall Meeting Abstracts, volume 1, page 1130, 2008.

[43] Steven R Beaupré and Ellen RM Druffel. Photochemical reactivity of ancient marine dissolved organic carbon. Geophysical Research Letters, 39(18), 2012.

[44] Boris P Koch, Matthias Witt, Ralph Engbrodt, Thorsten Dittmar, and Gerhard Kattner. Molecular formulae of marine and terrigenous dissolved organic matter detected by electrospray ionization fourier transform ion cyclotron resonance mass spectrometry. Geochimica et Cosmochimica Acta, 69(13):3299-3308, 2005.

[45] Elizabeth B Kujawinski, Rossana Del Vecchio, Neil V Blough, Geoffrey C Klein, and Alan G Marshall. Probing molecular-level transformations of dissolved organic matter: insights on photochemical degradation and protozoan modification of dom from electrospray ionization fourier transform ion cyclotron resonance mass spectrometry. Marine Chemistry, 92(1):23-37, 2004.

[46] D. R. Griffith, A. P. McNichol, L. Xu, F. A. McLaughlin, R. W. Macdonald, K. A. Brown, and T. I. Eglinton. Carbon dynamics in the western arctic ocean: insights from full-depth carbon isotope profiles of dic, doc, and poc. Biogeosciences, 9(3):1217-1224, 2012.

[47] R Ellen and M Druffel. Radiocarbon distributions in southern ocean dissolved and particulate organic matter. Geophysical research letters, 27(10):1495-1498, 2000 .

[48] Takayuki Tanaka, Shigeyoshi Otosaka, Masahide Wakita, Hikaru Amano, and Orihiko Togawa. Preliminary result of dissolved organic radiocarbon in the western north pacific ocean. Nuclear Instruments and Methods in Physics Research Section B: Beam Interactions with Materials and Atoms, 268(7):12191221, 2010.

[49] N. Jiao, G.J. Herndl, D.A. Hansell, R. Benner, G. Kattner, S.W. Wilhelm, D.L. Kirchman, M.G. Weinbauer, T. Luo, F. Chen, et al. Microbial production of recalcitrant dissolved organic matter: long-term carbon storage in the global ocean. Nature Reviews Microbiology, 8(8):593-599, 2010. 
[50] Kenneth Mopper, Xianliang Zhou, Robert J Kieber, David J Kieber, Richard J Sikorski, and Ronald D Jones. Photochemical degradation of dissolved organic carbon and its impact on the oceanic carbon cycle. Nature, 353(6339):60-62, 1991.

[51] C.A. Carlson, H.W. Ducklow, and A.F. Michaels. Annual flux of dissolved organic carbon from the euphotic zone in the northwestern sargasso sea. Nature, 371(6496):405-408, 1994.

[52] R.A. Jahnke. The global ocean flux of particulate organic carbon: Areal distribution and magnitude. Global Biogeochem. Cycles, 10:71-88, 1996.

[53] Lynne D Talley. Closure of the global overturning circulation through the indian, pacific, and southern oceans: Schematics and transports. Oceanography, 26(1):80-97, 2013.

[54] Richard A Jahnke. The global ocean flux of particulate organic carbon: Areal distribution and magnitude. Global biogeochemical cycles, 10(1):71-88, 1996.

[55] J. Arıstegui, C.M. Duarte, S. Agustı, M. Doval, X.A. Álvarez-Salgado, and D.A. Hansell. Dissolved organic carbon support of respiration in the dark ocean. Science, 298(5600):1967, 2002.

[56] David M Karl and Roger Lukas. The hawaii ocean time-series (hot) program: Background, rationale and field implementation. Deep Sea Research Part II: Topical Studies in Oceanography, 43(2):129-156, 1996.

[57] Deborah K Steinberg, Craig A Carlson, Nicholas R Bates, Rodney J Johnson, Anthony F Michaels, and Anthony H Knap. Overview of the us jgofs bermuda atlantic time-series study (bats): a decade-scale look at ocean biology and biogeochemistry. Deep Sea Research Part II: Topical Studies in Oceanography, 48(8):1405-1447, 2001.

[58] Jonathan H Sharp, Craig A Carlson, Edward T Peltzer, Dawn M Castle-Ward, Karen B Savidge, and Katherine R Rinker. Final dissolved organic carbon broad community intercalibration and preliminary use of doc reference materials. Marine Chemistry, 77(4):239-253, 2002.

[59] Raymond G Najjar, X Jin, F Louanchi, O Aumont, Ken Caldeira, Scott C Doney, J-C Dutay, M Follows, Nicolas Gruber, Fortunat Joos, et al. Impact of circulation on export production, dissolved organic matter, and dissolved oxygen in the ocean: Results from phase ii of the ocean carbon-cycle model intercomparison project (ocmip-2). Global Biogeochemical Cycles, 21(3), 2007.

[60] R. Francois, S. Honjo, R. Krishfield, and S. Manganini. Factors controlling the flux of organic carbon to the bathypelagic zone $\mathrm{f}$ the ocean. Global Biogeochemical Cycles, 16:art. no. 1087, 2002. 
[61] Robert M Key. Woce pacific ocean radiocarbon program. Radiocarbon, 38(3):415-423, 1996.

[62] A.E. Ingalls, S.R. Shah, R.L. Hansman, L.I. Aluwihare, G.M. Santos, E.R.M. Druffel, and A. Pearson. Quantifying archaeal community autotrophy in the mesopelagic ocean using natural radiocarbon. Proceedings of the National Academy of Sciences, 103(17):6442-6447, 2006.

[63] Bernadette M Sloyan and Stephen R Rintoul. The southern ocean limb of the global deep overturning circulation*. Journal of Physical Oceanography, 31(1):143-173, 2001.

[64] James E Bauer and Ellen RM Druffel. Ocean margins as a significant source of organic matter to the deep open ocean. Nature, 392(6675):482-485, 1998.

[65] James E Bauer, Ellen RM Druffel, Peter M Williams, David M Wolgast, and Sheila Griffin. Temporal variability in dissolved organic carbon and radiocarbon in the eastern north pacific ocean. Journal of Geophysical Research: Oceans (1978-2012), 103(C2):2867-2881, 1998.

[66] GAF Seber. Multivariate distributions. Multivariate Observations, pages 17-58, 1984.

[67] Bradley Efron and Robert J Tibshirani. An introduction to the bootstrap, volume 57. CRC press, 1994.

[68] E.R.M. Druffel, J.E. Bauer, P.M. Williams, S. Griffin, and D. Wolgast. Seasonal variability of particulate organic radiocarbon in the northeast pacific ocean. Journal of geophysical research, 101(C9):20543-20, 1996.

[69] J. Arsstegui, J.M. Gasol, C.M. Duarte, and GJ Herndl. Microbial oceanography of the dark oceans pelagic realm. Limnol. Oceanogr, 54(5):1501-1529, 2009.

[70] Mónica V Orellana and Dennis A Hansell. Ribulose-1, 5-bisphosphate carboxylase/oxygenase (rubisco): A long-lived protein in the deep ocean. Limnology and Oceanography, 57(3):826, 2012.

[71] Gordon T Taylor. Microbial degradation of sorbed and dissolved protein in seawater. Limnology and oceanography, 40(5):875-885, 1995.

[72] A.P. McNichol and L.I. Aluwihare. The power of radiocarbon in biogeochemical studies of the marine carbon cycle: insights from studies of dissolved and particulate organic carbon (doc and poc). Chemical reviews, 107(2):443-466, 2007.

[73] TI Eglinton and DJ Repeta. Organic matter in the contemporary ocean. Treatise on Geochemistry, 6:145-180, 2003. 
[74] James E Bauer, Clare E Reimers, Ellen RM Druffel, and Peter M Williams. Isotopic constraints on carbon exchange between deep ocean sediments and sea water. Nature, 373(6516):686-689, 1995.

[75] Peter A Raymond and James E Bauer. Use of ${ }^{14} \mathrm{C}$ and ${ }^{13} \mathrm{C}$ natural abundances for evaluating riverine, estuarine, and coastal doc and poc sources and cycling: a review and synthesis. Organic Geochemistry, 32(4):469-485, 2001.

[76] M.D. McCarthy, S.R. Beaupré, B.D. Walker, I. Voparil, T.P. Guilderson, and E.R.M. Druffel. Chemosynthetic origin of 14c-depleted dissolved organic matter in a ridge-flank hydrothermal system. Nature Geoscience, 4(1):32-36, 2010.

[77] J.W. Pohlman, J.E. Bauer, W.F. Waite, C.L. Osburn, and N.R. Chapman. Methane hydrate-bearing seeps as a source of aged dissolved organic carbon to the oceans. Nature Geoscience, 2010.

[78] CA Masiello and ERM Druffel. Black carbon in deep-sea sediments. Science, 280(5371):1911, 1998.

[79] T.A.J. Kuhlbusch. Black carbon and the carbon cycle. Science, 280(5371):1903, 1998.

[80] B.C. Cho and F. Azam. Major role of bacteria in biogeochemical fluxes in the ocean's interior. Nature, 332(6163):441-443, 1988.

[81] Ai Ning Loh, James E Bauer, and Ellen RM Druffel. Variable ageing and storage of dissolved organic components in the open ocean. Nature, 430(7002):877-881, 2004.

[82] D.J. Repeta and L.I. Aluwihare. Radiocarbon analysis of neutral sugars in high-molecular-weight dissolved organic carbon: Implications for organic carbon cycling. Limnology and oceanography, pages 1045-1053, 2006.

[83] A. Mahadevan. An analysis of bomb radiocarbon trends in the pacific. Marine chemistry, 73(3-4):273-290, 2001.

[84] E Tombari, G Salvetti, C Ferrari, and GP Johari. Kinetics and thermodynamics of sucrose hydrolysis from real-time enthalpy and heat capacity measurements. The Journal of Physical Chemistry B, 111(3):496-501, 2007.

[85] E.A. Laws, P.G. Falkowski, W.O. Smith, H. Ducklow, and J.J. McCarthy. Temperature effects on export production in the open ocean. Global Biogeochemical Cycles, 14(4):1231-1246, 2000.

[86] K.O. Buesseler, C.H. Lamborg, P.W. Boyd, P.J. Lam, T.W. Trull, R.R. Bidigare, J.K.B. Bishop, K.L. Casciotti, F. Dehairs, M. Elskens, et al. Revisiting carbon flux through the ocean's twilight zone. Science, 316(5824):567, 2007. 
[87] B.K. Swan, M. Martinez-Garcia, C.M. Preston, A. Sczyrba, T. Woyke, D. Lamy, T. Reinthaler, N.J. Poulton, E.D.P. Masland, M.L. Gomez, et al. Potential for chemolithoautotrophy among ubiquitous bacteria lineages in the dark ocean. Science, 333(6047):1296-1300, 2011.

[88] Dennis A Hansell and Craig A Carlson. Localized refractory dissolved organic carbon sinks in the deep ocean. Global Biogeochemical Cycles, 27(3):705-710, 2013.

[89] DA Siegel, KO Buesseler, SC Doney, SF Sailley, MJ Behrenfeld, and PW Boyd. Global assessment of ocean carbon export by combining satellite observations and food-web models. Global Biogeochemical Cycles, 2014.

[90] Ellen RM Druffel and James E Bauer. Radiocarbon distributions in southern ocean dissolved and particulate organic matter. Geophysical Research Letters, 27(10):1495-1498, 2000.

[91] Chiara Santinelli, Luciano Nannicini, and Alfredo Seritti. Doc dynamics in the meso and bathypelagic layers of the mediterranean sea. Deep Sea Research Part II: Topical Studies in Oceanography, 57(16):1446-1459, 2010.

[92] H. D. Holland. The Chemistry of the Atmosphere and Oceans. John Wiley \& Sons, New York, 1978.

[93] J. I. Hedges and R. G. Keil. Sedimentary organic matter preservation: an assessment and speculative synthesis. Marine Chemistry, 49:81-115, 1995.

[94] R. A. Berner. The Phanerozoic Carbon Cycle.

[95] L. M. Mayer. Surface-area control of organic-carbon accumulation in continental-shelf sediments. Geochimica et Cosmochimica Acta, 58:1271-1284, 1994.

[96] B. Ransom, R.H. Bennett, R. Baerwald, and K. Shea. TEM study of in situ organic matter on continental margins: occurrence and the "monolayer" hypothesis. Marine Geology, 138:1-9, 1997.

[97] C. Klaas and D.E. Archer. Association of sinking organic matter with various types of mineral ballast in the deep sea: Implications for the rain ratio. Global Biogeochem. Cycles, 16(4):1116, 2002.

[98] R.A. Armstrong, C. Lee, J.I. Hedges, S. Honjo, and S.G. Wakeham. A new, mechanistic model for organic carbon fluxes in the ocean based on the quantitative association of POC with ballast minerals. Deep-Sea Research Part II, 49(1-3):219-236, 2001.

[99] R. A. Armstrong, C. Lee, J. I. Hedges, S. Honjo, and S. G. Wakeham. A new, mechanistic model for organic carbon fluxes in the ocean based on the quantitative association of POC with ballast materials. Deep-Sea Research II, 49:219-236, 2002. 
[100] Christine Klaas and David E Archer. Association of sinking organic matter with various types of mineral ballast in the deep sea: Implications for the rain ratio. Global Biogeochemical Cycles, 16(4):63-1, 2002.

[101] M. Kennedy, M. Droser, L. M. Mayer, D. Pevear, and D. Mrofka. Late Precambrian oxygenation; inception of the clay mineral factory. Science, 311:14461449, 2006.

[102] N.M. Mahowald, A.R. Baker, G. Bergametti, N. Brooks, R.A. Duce, T.D. Jickells, N. Kubilay, J.M. Prospero, and I. Tegen. Atmospheric global dust cycle and iron inputs to the ocean. Global Biogeochem. Cycles, 19(4), 2005.

[103] Burdige. Biogeochemistry of Marine Sediments. 2006. pg 163.

[104] J. J. Middelburg. A simple rate model for organic matter decomposition in marine sediments. Geochimica et Cosmochimica Acta, 53:1577-1581, 1989.

[105] B. B. Jorgensen. A comparison of methods for the quantification of bacterial sulfate reduction in coastal marine sediments. II. Calculation from mathematical models. Geomicrobiology Journal, 1:29-47, 1978.

[106] R. G. Keil, E. Tsamakis, C.B. Fuh, J.C. Giddings, and J. I. Hedges. Mineralogical and textural controls on the organic composition of coastal marinesediments - hydrodynamic separation using SPLITT-fractionation. Geochimica et Cosmochimica Acta, 58:879-893, 1994.

[107] R. G. Keil, D. B. Montlucon, F. G. Prahl, and J. I. Hedges. Sorptive preservation of labile organic-matter in marine-sediments. Nature, 370:549-552, 1994.

[108] L. M. Mayer. Relationships between mineral surfaces and organic-carbon concentrations in soils and sediments. Chemical Geology, 114:347-363, 1994.

[109] E. T. Premuzic, C. M. Benkovitz, J. S. Gaffney, and J. J. Walsh. The nature and distribution of organic matter in the surface sediments of world oceans and seas. Organic Geochemistry, 4:63-77, 1982.

[110] R. A. Berner. Early Diagenesis: A Theoretical Approach. Princeton University Press, Princeton, N. J., 1980.

[111] J. I. Hedges, J. A. Baldock, Y. Gelinas, C. Lee, M. Peterson, and S. G. Wakeham. Evidence for non-selective preservation of organic matter in sinking marine particles. Nature, 409:801-804, 2001.

[112] Jens Kallmeyer, Robert Pockalny, Rishi Ram Adhikari, David C Smith, and Steven DHondt. Global distribution of microbial abundance and biomass in subseafloor sediment. Proceedings of the National Academy of Sciences, 109(40):16213-16216, 2012. 
[113] Frank M Stewart and Bruce R Levin. Partitioning of resources and the outcome of interspecific competition: a model and some general considerations. American Naturalist, pages 171-198, 1973.

[114] B.P. Boudreau, C. Arnosti, B.B. Jørgensen, and D.E. Canfield. Comment on "Physical Model for the Decay and Preservation of Marine Organic Carbon". Science, 319:1616, 2008.

[115] Y.A. Vetter, J.W. Deming, P.A. Jumars, and B.B. Krieger-Brockett. A predictive model of bacterial foraging by means of freely released extracellular enzymes. Microbial Ecology, 36:75-92, 1998.

[116] M Kamer and F Rassoulzadegan. Extracellular enzyme activity: indications for high short-term variability in a coastal marine ecosystem. Microbial ecology, 30(2):143-156, 1995.

[117] Xiaoling Ding and Susan M Henrichs. Adsorption and desorption of proteins and polyamino acids by clay minerals and marine sediments. Marine Chemistry, 77(4):225-237, 2002.

[118] EL Cussler. Diffusion: Mass transfer in fluid systems. Cambridge Univ Pr, 1997.

[119] T.C. Farrar. Introduction to pulse NMR spectroscopy. Farragut Press Chicago, 1997.

[120] P. Stilbs. Fourier transform pulsed-gradient spin-echo studies of molecular diffusion. Progress in nuclear magnetic resonance spectroscopy, 19(1):1-45, 1987.

[121] S.J. Gibbs, E.N. Lightfoot, and T.W. Root. Protein diffusion in porous gel filtration chromatography media studied by pulsed field gradient NMR spectroscopy. The Journal of Physical Chemistry, 96(18):7458-7462, 1992.

[122] D.M. Jung, J.S. De Ropp, and S.E. Ebeler. Application of pulsed field gradient NMR techniques for investigating binding of flavor compounds to macromolecules. J. Agric. Food Chem, 50(15):4262-4269, 2002.

[123] Carl-Erik Danielsson. Molecular weight of alpha-amylase. Nature, 160:899, 1947.

[124] Xu-Chen Wang and Cindy Lee. Adsorption and desorption of aliphatic amines, amino acids and acetate by clay minerals and marine sediments. Marine Chemistry, 44(1):1-23, 1993. 